\title{
CEPREMAP
}

BIBLIOYHEQUE

142, rue du Chevaloret

75013 PARIS

Tél. : 40778420

\section{NONLINEAR DIFFERENCE EQUATIONS}

\section{BIFURCATIONS AND CHAOS : \\ AN INTRODUCTION}

\author{
by Jean-Michel Grandmont ${ }^{\star}$
}

Juin 1988

$N^{*} 8811$

* This work is supported by a grant of the National Science Foundation at the Institute for Mathematical Studies in the Social Sciences, Stanford University, Stanford, California. Financial support from the Suntory Toyota International Centre for Economics and Related Disciplines, London School of Economics, London, is also gratefully acknowledged.

I greatly benefited from the kind advice and outstanding expertise of Alain CHENCINER, in particular from a two days course he gave on the Hopf bifurcation at the Groupement de Recherche en Economie Quantitative et Econométrie, Ecole des Hautes Etudes en Sciences Sociales, in Marseille in the fall of 1986. I wish to thank him very much here.

** CNRS and CEPREMAP, 142, rue du Chevaleret, 75013 PARIS, France. 


\title{
NONLINEAR DIFFERENCE EQUATIONS \\ BIFURCATIONS AND CHAOS : AN INTRODUCTION
}

\author{
A B STRACT
}

The aim of these lecture notes is to present a few mathematical facts about the bifurcations of nonlinear difference equations, in a compact form that might be useable by economic theorists. These notes should be part of the mathematical appendices of a forthcoming book "Market Psychology and Business Cycles" that will be eventually published by Oxford University Press.

The material of these lectures was presented as a short course at the Summer Workshop in Economic Theory at Stanford University (IMSSS) in the summer of 1987. The comments and suggestions of the participants are gratefully acknow ledged.

Keywords : Economic Fluctuations

JEL code : 131

$$
\text { -ニーニーニーニーニーニーニーニーニーニーニー }
$$

\begin{tabular}{lll} 
CONTENTS & A. PRELIMINARIES & $P$. \\
\hline & B. LINEAR AND NONLINEAR DIFFERENCE EQUATIONS & $P .13$ \\
& C. LOCAL BIFURCATIONS & $P .38$ \\
& D. GLOBAL BIFURCATIONS AND CHAOS & $P .82$
\end{tabular}

EQUATIONS AUX DIFFERENCES NON LINEAIRES

BIFURCATIONS ET CHAOS : UNE INTRODUCTION

\section{RESUNE}

Le but de ces notes est de présenter que lques résultats mathématiques concernant les bifurcations d'équations aux différences non linéaires, dans un format compact qui puisse être utilisé par les théoriciens de l'économie. Ces notes devraient constituer une partie des appendices mathématiques d'un ouvrage à paraitre "Market Psychology and Business Cycles" qui sera publié par Oxford University Press.

Le contenu de ces notes a été présenté dans un mini-cours à l'Atelier d'été en théorie économique, à l'Université de Stanford (IMSSS), durant l'été 1987. Les commentaires et suggestions des participants m'ont été très utiles.

Mots clef : Fluctuations économiques

Code JEL : 131 


\section{A. PRELIMINARIES}

We state first a few more or less elementary facts about matrices and differentiable maps, that are used repeatedly in the sequel.

We recall that $R^{m}$ is the set of all $\mathrm{m}$-tuples of real numbers. A "point" or a "vector" of $R^{m}$ is $x=\left(x_{1}, \ldots, x_{m}\right)$; the number $x_{i}$ is the $i$-th coordinate of the vector. Vectors $x, y$ are added coordinatewise

$$
x+y=\left(x_{1}, \ldots, x_{m}\right)+\left(y_{1}, \ldots, y_{m}\right)=\left(x_{1}+y_{1}, \ldots, x_{m}+y_{m}\right) .
$$

If $\alpha$ is a real number, the product $\alpha x$ is the vector $\left(\alpha x_{1}, \ldots, \alpha x_{m}\right) . R^{m}$ is then an $\mathrm{m}$-dimensional real vector space. Its standard basis is the collection of vectors $e_{1}, \ldots, e_{m}$, in which for each $i=1, \ldots, m, e_{i}$ is the vector of coordinates $e_{i j}=\delta_{i j}, j=1, \ldots, m$, where $\delta_{i j}$ is the Kronecker function, that is $\delta_{i j}=0$ if $i \neq j$ and $i$ if $i=j$. Any vector $x=\left(x_{1}, \ldots, x_{m}\right)$ has then $a$ unique representation as a linear combination of the vectors $e_{i}$ of the standard basis, that is $x={ }_{i} x_{i} e_{i}$. A norm is a real valued function 11.11 defined on $R^{m}$, with $\|x\| \geq 0$, such that $\|\alpha x\|=|\alpha|\|x\|, \||x+y||\leqq||x||+||y| \mid$, and and $\|x\|=0$ if and only if $x=0$. The Euclidean norm will be denoted $|x|=\left(\Sigma_{i} x_{i}^{2}\right)^{1 / 2}$.

\section{A.1. Matrix algebra}

A square matrix of dimension $m$, i.e. a collection of $m^{2}$ real numbers $A=\left[a_{i j}\right]$, where $i=1, \ldots, m$ stands for the index of the $i$-th row of the matrix, and $j=1, \ldots, m$ stands for its $j$-th column, defines a linear 
transformation (or map) $T$ from $R^{m}$ into itseif, that associates to every vector $x=\left(x_{1}, \ldots, x_{m}\right)$ a new vector $x^{\prime}=T x$ of coordinates $x_{i}^{\prime}=\varepsilon_{j} a_{i j} x_{j}$. for $i=1, \ldots, m$, or in matrix notation, $x^{\prime}=A x$. Then if $e, \ldots, e$ is the standard basis of $R^{m}$, the vector represented by the $j$-th column of $A, i . e$. $a^{j}=\left(a_{1 j}, \ldots, a_{m j}\right)$, is the image of $e_{j}$ by $T$ (or $\left.A\right)$, that is $a^{j}=T e_{j}=A e_{j}$. The image $x^{\prime}=$ Ax of any vector $x=\left(x_{1}, \ldots, x_{m}\right)$ is the linear combination of the vectors $a^{j}$, with weights $x_{j}$ :

$$
\left.x^{\prime}=A\left(\Sigma_{j} x_{j} e\right)_{j}\right)=\Sigma_{j} x_{j} a^{j}
$$

Conversely, any linear transformation $T$ from $R^{m}$ into itself can be (uniquely) represented by the matrix $A={ }_{\left[a_{i j}\right.}{ }$, in the standard basis, where the $j$-th column $a^{j}=\left(a_{1 j}, \ldots, a_{m j}\right)$ of the matrix $A$ is the image by $T$. of the vector $e_{j}$. It follows from these remarks that a matrix $A$ is invertible if and only if the corresponding linear transformation $T$ in onto (i.e. the image of $R^{m}$ by $T$ is $R^{m}$ itself), or equivalently, if and only if the $m$ vectors $a^{j}$ are linearly independent (i.e. $\sum_{j} \alpha_{j} a^{j}=0$ implies $\alpha_{j}=0$ for all $j$ ).

A given linear transformation $T$ of $R^{m}$ into itse if has different equivalent matrix representations, according to which basis of $R^{m}$ is chosen. Consider a new basis of $R^{m}$, i.e. a collection of $m$ vectors $\bar{e}_{1}, \ldots, \overline{\mathrm{e}}_{\mathrm{m}}$, that are linearly independent. Let $\left(p_{1 j}, \ldots, p_{m j}\right)$ be the coordinates of $\bar{e}_{j}$ in the standard basis, and $P$ stand for the matrix of which the $j$-th column is $\bar{e}_{j}$, i.e. $P=\left[p_{i j}\right]$. We know from the previous paragraph that $P$ has an inverse $P^{-1}$. A vector of $R^{m}$ of which the coordinates in the old (standard) basis are $x=\left(x_{1}, \ldots, x_{m}\right)$, has coordinates $y=\left(y_{1}, \ldots, y_{m}\right)$ in the new basis. That is, 
this vector can be (uniquely) expressed as a linear combination of the vectors $\bar{e}_{j}$ of the new basis, with weights $y_{j}$, i.e. $\Sigma_{j} y_{j} \bar{e}_{j}$. The relationship between new and old coordinates is obtained from the vector equalities

$$
\Sigma_{i} x_{i} e_{i}=\Sigma_{j} y_{j} \bar{e}_{j}=\Sigma_{j} y_{j}\left(\Sigma_{i} p_{i j} e_{i}\right)
$$

which imply $x_{i}=E_{j} p_{i j} y_{j}$ for all $i$, or in matrix notation, $x=P y$, $y=P^{-1} x$.

A given linear transformation $T$ is represented, in matrix notation, by the map $x+x^{\prime}=A x$ in the standard basis, and by $y \rightarrow y^{\prime}=B x$ in the new basis. Analytically, the matrix $B$ is obtained from $A$ by making the change of variables $x=P y$, which yields $B=P^{-1} A P$. Here again, the $j$-th column of $B$ represents the coordinates, in the new basis, of the image of $\bar{e}_{j}$ by $T$.

A linear transformation $T$ of $R^{m}$ into itself may thus be given a convenient matrix representation, by choosing an appropriate basis. The remainder of this section is devoted to such a matrix representation, the real canonical (or Jordan) form of $T$.

We look first at the circumstances ensuring that $T$ has a block diagonal matrix representation. Let $E_{1}, \ldots, E_{\Gamma}$ be a collection of (1inear) subspaces of $R^{m}$, i.e. each $E_{h}$ is a subset of $R^{m}$ that is closed under the operations of addition and scalar multiplication : if $x, y$ are vectors of $E_{h}$ and $\alpha$ a real number, then $x+y$ and $\alpha x$ be long also to $E_{h}$. Assume that any vector $x$ of $R^{m}$ has a unique representation of the form $x=x_{1}+\ldots+x_{r}$, in which $x_{h}$ is in $E_{h}$ for each $h$. We say then that $R^{m}$ is the direct sum of the linear subspaces. Assume further that each subspace $E_{h}$ is invariant by $T$, i.e. if $x$ belongs to $E_{h}$, then $T x$ is also in $E_{h}$. Choose now a basis for each $E_{h}$, and take the 
union of the basis elements of the $E_{h}$ to obtain a basis for $R^{m}$. In that basis, $T$ has the block diagonal form

$$
B=\operatorname{diag}\left(B_{1}, \ldots, B_{r}\right\rangle=\left[\begin{array}{cccc}
B_{1} & & \\
& \ddots & \\
& & B_{r}
\end{array}\right]
$$

This means that the matrices $B_{h}$ are put together corner-to-corner diagonally as indicated, all other entries in B being zero (we adopt the convention that the blank entries in a matrix are zeros). Each matrix $B_{h}$ represents in fact the restriction $T_{h}$ of $T$ to the invariant subspace $E_{h}$.

Conversely, assume that $R^{m}$ has a basis in which $T$ has a matrix representation of the above block diagonal form. Let $E_{h}$ be the linear subspace spanned by the vectors of the basis, the images of which are the columns of the matrix $B$ associated to the submatrix $B_{h} \cdot$. Then $E_{h}$ is invariant by $T$, and $R^{m}$ is the direct sum of the $E_{h}$. To sum up,

Proposition A.1.1. Let $T$ be a linear transformation of $R^{m}$ into itse if. T has a block diagonal matrix representation if and only if there exists a collection of linear subspaces $E_{h}$ of $R^{m}, h=1, \ldots, r$, such that 1$)$ each $E_{h}$ is invariant by $T$, and 2) $R^{m}$ is the direct sum of the $E_{h}$.

The real canonical matrix representation of $T$ is obtained when the invariant subspaces $E_{h}$ are taken to be the "real generalized eigenspaces" of T (to be defined short $7 y$ ), with an appropriate basis. 


\section{Distinct eigenvalues.}

Let $A$ be the matrix representation of $T$ in the standard basis. We recall that an eigenvalue $\lambda$ of $T$ is a (possibly complex) number such that $A E=\lambda E$ has a nonzero solution $E$, where $E$ is an m-dimensional vector, the coordinates of which may be complex numbers. Such a nonzero solution $\varepsilon$ is called an eigenvector (of $T$ ) corresponding to $\lambda$. The set of (zero and nonzero) solutions $\varepsilon$ of $A \xi=\lambda \xi$ is the eigenspace corresponding $\lambda$, and is noted $E(\lambda)$.

$E(\lambda)$ is a complex vector space, i.e. if $E, \eta$ are in $E(\lambda)$ and $z$ is a complex number, then $\xi+\eta$ and $z E$ are also in $E(\lambda)$. If, however, $\lambda$ is real, the equation $A E=\lambda E$ has a nonzero solution $x$ in $P^{m}$, a real eigenvector. The real eigenspace $F(\lambda)$ is then the set of all real solutions of $A E=\lambda E . F(\lambda)$ is a linear subspace of $R^{m}$, and $E(\lambda)$ is its "complexification" : every $E$ in $E(\lambda)$ is of the form $u+i v$, where $u, v$ belong to $F(\lambda)$ and $i=\sqrt{-1}$.

If the eigenvalue is nonreal, i.e. $\lambda=a+i b$ with $b \neq 0$, then every eigenvector $\xi$ corresponding to $\lambda$ must be nonreal, i.e. have the form $u+i v$ where $u, v$ are vectors of $R^{m}$ and $v \neq 0$. In that case, the complex conjugate of $\lambda, i . e \cdot \bar{\lambda}=a-i b$, is also an eigenvalue, and $\varepsilon=u+i v$ belongs to $E(\lambda)$ if and only if its complex conjugate $\bar{\xi}=u$. - iv belongs to $E(\bar{\lambda})$. This follows from the fact that $A E=\lambda E$ is equivalent, through complex conjugation, to $\overline{A E}=\bar{\lambda} \bar{\varepsilon}$. Consider next the set $F(\lambda)$ of all vectors of $R^{m}$ that lie in $E(\lambda)+E(\bar{\lambda})$, i.e. that are of the form $\xi+\eta$, where $\varepsilon$ and $\eta$ belong to $E(\lambda)$ and $E(\bar{\lambda})$, respectively. If $E=u+$ iv belongs to $E(\lambda)$ or $E(\bar{\lambda})$, then $u, v$ are in $F(\lambda)$, since $u=(\varepsilon+\bar{\varepsilon}) / 2$ and $v=(\varepsilon-\bar{\varepsilon}) / 2 i$. The set $F(\lambda)$ is a linear subspace of $R^{m}$, and one can verify that $E(\lambda)+E(\bar{\lambda})$ is its complexification. 
By a slight abuse of language, we call $F(\lambda)$ the real eigenspace corresponding to $\lambda($ or $\bar{\lambda}$ ), although it does not contain any eigenvector.

It is clear that every eigenspace $E(\lambda)$ or $F(\lambda)$ is invariant by $T$.

Eigenvalues are found by solving, in the complex plane, the polynomial equation

$$
p(\lambda) \equiv \operatorname{Det}(A-\lambda I)=0
$$

where $I$ is the $m$-dimensional identity matrix, and $p(\lambda)$ is the determinant of $A-\lambda I$. The expression $p(\lambda)$ is called the characteristic polynomial of $T$ (or A). Its degree is $m$. T has distinct eigenvalues if the characteristic equation $p(\lambda)=0$ has $m$ distinct roots. The following result shows that $T$ has a particularly simple matrix representation when its eigenvalues are distinct.

Theorem A.1.2. Let $T$ be a linear transformation of $R^{m}$ into itself, with distinct eigenvalues. Then every eigenspace $E(\lambda)$ is one-dimensional. Let the real eigenvalues be $\lambda_{1}, \ldots, \lambda_{r}$, and the nonreal eigenvalues be $\mu_{1}, \bar{\mu}_{1}, \ldots, \mu_{s}$, $\bar{\mu}_{s}$, with $\mu_{k}=a_{k}+i b_{k}, b_{k}>0$ for all $k$, and $r+2 s=m$. Then $R^{m}$ is the direct sum of the real eigenspaces $F\left(\lambda_{1}\right), \ldots, F\left(\lambda_{r}\right), F\left(\mu_{1}\right), \ldots, F\left(\mu_{s}\right)$. The dimension of each $F\left(\lambda_{h}\right)$ is one. while the dimension of each $F\left(\mu_{k}\right)$ is two. Each of these eigenspaces is invariant by $T$. Moreover, $R^{m}$ has a basis in which $T$ has the following block diagonal matrix representation 


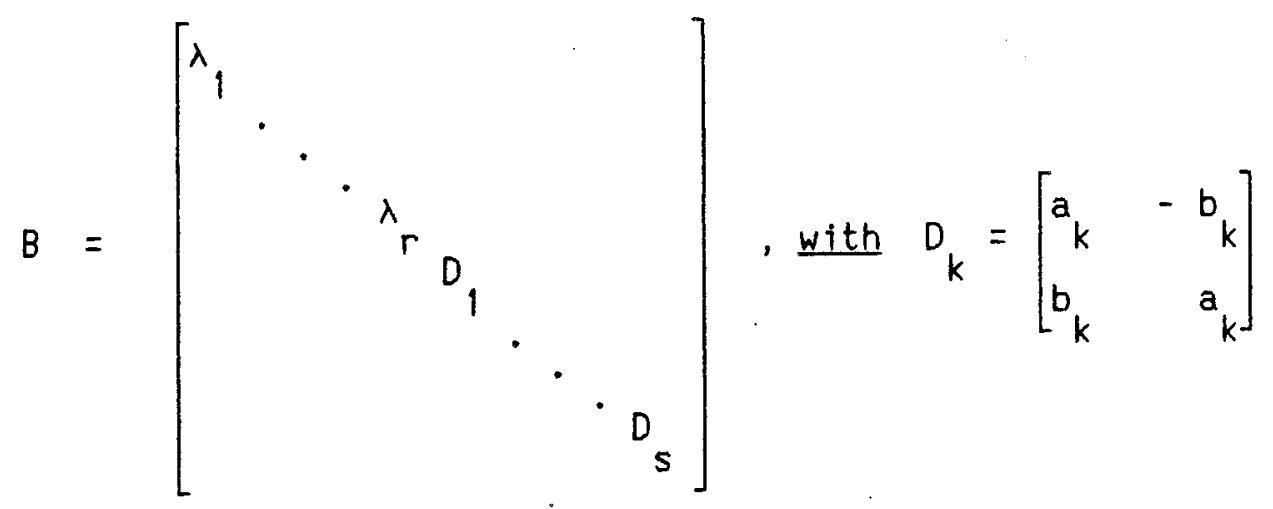

for $k=1, \ldots, s$.

Remark A.1.3. In the theorem, the basis is obtained in the following manner. The first $r$ elements are real eigenvectors $x_{h}$ in $F\left(\lambda_{h}\right), h=1, \ldots, r$. For each $k=1, \ldots, s,\left\langle v_{k}, u_{k}\right)$ is a basis for $F\left(\mu_{k}\right)$, where $u_{k}+i v v_{k}$ is an eigenvector in $E\left(\mu_{k}\right)$. The whole basis is actually $\left\langle x_{q}, \ldots, x_{r}, v_{1}, u_{1}, \ldots, v_{s}, u_{s}\right\rangle$. For details, see Hirsch and Smale (1974), Chapter 4, Section 2.

\section{Multiple eigenvalues}

We look now at the case in which $T$ may have multiple eigenvalues. The characteristic polynomial can always be factorized as

$$
p(\lambda) \equiv\left(\lambda_{1}-\lambda\right)^{n_{1}} \ldots\left(\lambda_{q}-\lambda\right)^{n_{a}}
$$

where the $\lambda_{i}$ are the distinct eigenvalues of $T$, and $n_{i}$ is the multiplicity of $\lambda_{i}$. One has $n_{1}+\ldots+n_{q}=m$, and $T$ has multiple eigenvalues if some $n_{i} \geq 2$

In such a case, one has to introduce the notions of "generalized" eigenvectors and eigenspaces. Let $\lambda$ be an eigenvalue of $T$, and $n$ its 
multiplicity. The generalized eigenspace $E^{\star}(\lambda)$ corresponding to $\lambda$ is the space of $m$-dimensional complex vectors $\varepsilon$ that solve $(A-\lambda I)^{n} \xi=0$. If $\varepsilon$ is in $E^{\star}(\lambda)$ and $E \neq 0$, then $E$ is a generalized eigenvector. Of course if $n=1$, these notions reduce to the usual concepts of an eigenspace and an eigenvector.

The arguments employed above to define real eigenvectors or eigenspaces apply equally well here. If $\lambda$ is real, the space of all vectors in $E^{\star}(\lambda)$ that are real, is the generalized real eigenspace $F^{\star}(\lambda)$ corresponding to $\lambda$. If $x$ is in $F^{*}(\lambda)$ and $x \neq 0$, it is a generalized real eigenvector. If $\lambda$ is nonreal, the the generalized real eigenspace $F^{\star}(\lambda)$ (corresponding to $\lambda$ ) is the set of all real vectors of $E^{\star}(\lambda)+E^{\star}(\bar{\lambda})$. Here again, there is a slight abuse of language in such a definition, since there are no real generalized eigenvectors when $\lambda$ is nonrea 1 .

One can verify that the dimension of $E^{*}(\lambda)$ is $n$; the dimension of $F^{\star}(\lambda)$ is thus $n$ when $\lambda$ is real, $2 n$ otherwise. It is easy to see that $E^{\star}(\lambda)$ and $F^{*}(\lambda)$ are invariant by $T$.

Theorem A.1.4. Let $T$ be a linear transformation of $R^{m}$ into itself. Let $\lambda_{1}, \ldots, \lambda_{r}$ be its distinct real eigenvalues, with $\lambda_{h}$ having multiplicity $n_{h}$, and $\mu_{1}, \bar{\mu}_{1}, \ldots, \mu_{s}, \bar{\mu}_{s}$ its distinct nonreal eigenvalues, with $\mu_{k}=a_{k}+i b_{k}$, $b_{k}>0$, having multiplicity $n_{k}$. Then $R^{m}$ is the direct sum of the generalized real eigenspaces $F^{*}\left(\lambda_{1}\right), \ldots, F^{*}\left(\lambda_{r}\right), F^{\star}\left(\mu_{1}\right), \ldots, F^{*}\left(\mu_{s}\right)$. The dimension of $F^{*}\left(\lambda_{h}\right)$ is $n_{h}$, while the dimension of $F^{*}\left(\mu_{k}\right)$ is $2 n_{k}$. Each of these eigenspaces is invariant by $T$. 
Moreover, $R^{m}$ has a basis in which $T$ has the following block diagonal matrix representation

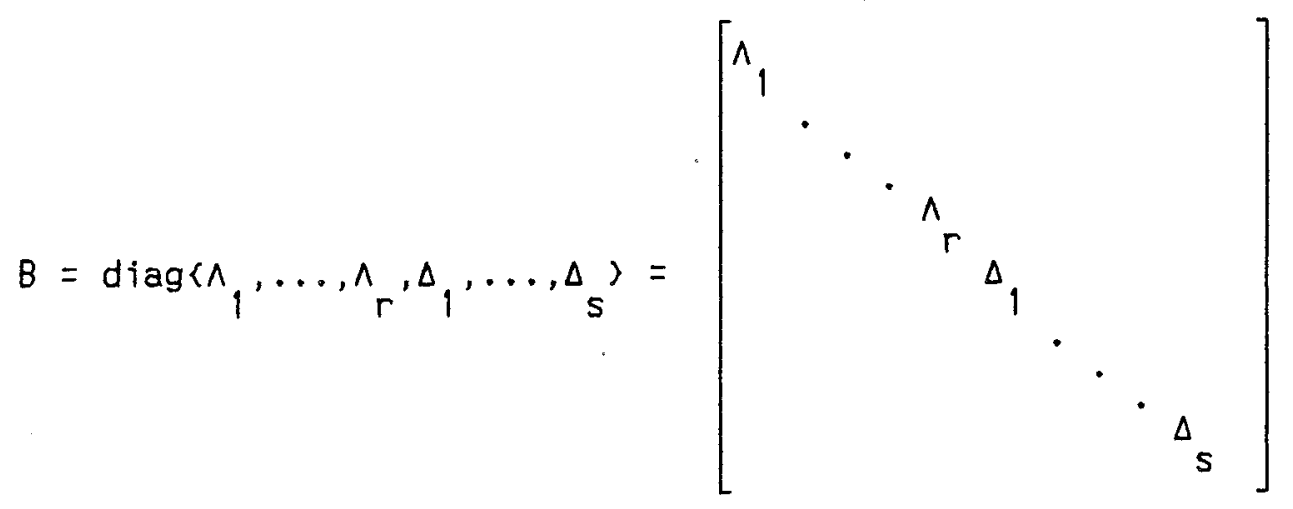

where

1) for each $h=1, \ldots, r$, the matrix $\wedge_{h}$ is $n_{h}$-dimensional block diagona?, every block on the diagonal being of the form

$$
\left[\begin{array}{lll}
\lambda_{h} & & \\
\ddots & \ddots & \\
& \lambda_{h}
\end{array}\right] \quad \text { or } \quad \lambda_{h}
$$

The number of these blocks is equal to the dimension of the eigenspace $E\left(\lambda_{h}\right)$.

2) for each $k=1, \ldots, s$, the matrix $\Delta_{k}$ is $2 n_{k}$-dimensional block diagonal, every block on the diagonal being of the form

$$
\left[\begin{array}{lll}
D_{k} & & \\
I_{2} & \ddots & \\
& I_{2} & D_{k}
\end{array}\right] \quad \text { or } \quad D_{k}
$$


with $D_{k}=\left[\begin{array}{cc}a_{k} & -b_{k} \\ b_{k} & a_{k}\end{array}\right]$ and $I_{2}$ the two-dimensional identity matrix. The number of these blocks is equal to the dimension of the eigenspace $E\left(\mu_{k}\right)$.

The above block diagonal matrix representation is called the real canonical (or Jordan) form of $T$. The block diagonal canonical representation is unique, up to the order of the blocks on the diagonal. Of course, it reduces to the matrix representation of Theorem A.1.2 in the case of distinct eigenvalues, i.e. when $n_{h}=n_{k}=1$ for all $h$ and $k$. As an incidental remark, the above theorem implies that a matrix $A$ is invertible if and only if none of its eigenvalues is equal to zero.

Remark A.1.5. In the Theorem, the basis is the union of bases for each generalized real eigenspace $F^{\star}\left(\dot{\lambda}_{1}\right), \ldots, F^{\star}\left(\lambda_{r}\right), F^{*}\left(\mu_{1}\right), \ldots, F^{*}\left(\mu_{s}\right)$, in that order. For $F^{\star}\left(\mu_{k}\right)$, the basis is of the form $\left\langle v_{1}, u_{1}, \ldots, v_{s}, u_{s}\right\}$, where $\left.\left\{u_{1}+i v_{1}, \ldots, u_{s}+i v\right\rangle_{s}\right\rangle$ is a basis for $E^{\star}\left(\mu_{k}\right)$. For details, see Hirsch and Smale (1974, Chapter 6, Section 4, and Appendix III).

\section{A.2. The implicit function theorem}

Let $G$ be a map from an open subset $W$ of $R^{m}$ into $R^{m}$. We say that $G$ is $C^{r}$ with $r \geq 1$, if $G$ has continuous partial derivatives of every order $h=1, \ldots, r$ at each point of $W . G$ is an homeomorphism if it is continuous and one to one, and if its inverse $G^{-1}$ (a map from $G(W)$ onto $W$ ) is also continuous. 
$G$ is a $C^{r}$ diffeomorphism if it is $C^{r}$ and one to one, and if its inverse is also $C^{r}$.

If $G$ is $C^{\prime}$, the matrix of partial first derivatives at a point $x$ of $W$ is called the Jacobian matrix of $G$ at $x$, and is noted $D G(x)$.

The following result gives conditions ensuring that a $c^{r}$ map is locally a diffeomorphism.

Theorem A.2.1. (Inverse function theorem). Let $G$ be a $C^{r}$ map from an open set $W$ in $R^{m}$ into $R^{m}$, with $r \geq 1$. Let $\times$ be a point of $W$ and assume that the Jacobian matrix $D G(x)$ is invertible. Then $x$ has an open neighbourhood $U$ such that the restriction of $G$ to $U$ is a $C^{r}$ diffeomorphism onto the open set $G(U)$.

Under the conditions of the theorem, we shall say that $G^{-1}: G(U)+U$ is a local inverse of $G$. Application of the inverse function theorem permits then to see when an implicit equation $F(x, y)=c$ can be solved and generate, at least locally, a functional relation of the form $y=G(x)$.

Theorem A.2.2. (Implicit function theorem). Let $W$ be an open set in $R^{m} \times R^{p}$, and $F$ a $C^{\Gamma}$ map from $W$ into $R^{p}$, that is $(x, y) \rightarrow F(x, y)$ where $x$ and $y$ are vectors of $R^{m}$ and $R^{p}$, respectively. Let $\left(x_{0}, y_{0}\right)$ in $W$ be such that $F\left(x_{0}, y_{0}\right)=c$ and suppose that the Jacobian matrix of the map $F\left(x_{0},.\right)$ is invertible at $y=y_{0}$ 
Then there are open sets $U$ and $V$ in $R^{m}$ and $R^{p}$, respectively, with $x_{0}$ in $U, y_{0}$ in $V$ and $U \times V$ contained in $W$, and a unique $C^{r}$ map $G: U+V$, such that

$$
F(x, G(x))=c
$$

for all $x$ in $U$, and moreover, $F(x, y) \neq c$ if $x$ is in $U, y$ in $V$ and $y \neq G(x)$.

\section{Notes on the Iiterature}

The material of Section A.l can be found in any textbook on linear algebra. The presentation here is adapted from Hirsch and Smale (1974), see also $\mathrm{Pal}$ is and de Melo (1982, chap. 2.2). For a proof of the inverse and the implicit function theorems, see Hirsch and Smale (1974, Appendix IV). 


\section{B. LINEAR AND NONLINEAR DIFFERENCE EQUATIONS}

We shall be concerned in this appendix, and in the next two ones, with the qualitative behaviour of the trajectories generated by a difference equation of the form

$(B .1)$

$$
x_{n+1}=G\left(x_{n}\right)
$$

in which $G$ is a map from an open subset $U$ of $R^{m}$ into $R^{m}$. Our first specific task, which will be carried out presently, is to proceed to such a study in the immediate vicinity of a fixed point of $G$ (a)so called a stationary state, an equilibrium point), i.e. an element $\bar{x}$ of $U$ such that $\bar{x}=G(\bar{x})$. It will be seen in particular that if $G$ is continuously differentiable, the trajectories generated by $(B .1)$ that are close enough to $\bar{x}$, are generally similar to those that are associated to the "linearized" version

$$
x_{n+1}-\bar{x}=D G(\bar{x})\left(x_{n}-\bar{x}\right)
$$

in which $D G(\bar{x})$ stands for the Jacobian matrix of $G$, evaluated at the fixed point.

A frequently encountered situation giving rise to a formulation such as $(B .1)$ is the following one. Suppose that the successive states $y_{n}$ of a given (physical, social) system must satisfy

$$
F\left(y_{n+1}, y_{n}, \ldots, y_{n-N}\right)=0
$$

in which each $y$ is a vector of $R^{D}$, and $F$ is a $C^{r}$ map from an open set $W$ of $R^{p(N+2)}$ into $R^{p}$. Let $\bar{y}$ be a stationary state, i.e. a vector of $R^{p}$ such that $F(\bar{y}, \ldots, \bar{y})=0$, and assume that the Jacobian matrix of the map 
$y \rightarrow F(y, \bar{y}, \ldots, \bar{y})$, evaluated at $\bar{y}$, is invertible. The Implicit Function

Theorem A.2.2 implies that (B.3) can then be solved in $y_{n+1}$ in a neighbourhood of $\bar{y}$. That is, there exist open sets $U$ in $R^{p(N+1)}$ and $V$ in $R^{p}$, containing $(\bar{y}, \ldots, \bar{y})$ and $\bar{y}$ respectively, and a unique $C^{r}$ map $H: U \rightarrow V$ such that $\left(y_{n+1}, y_{n}, \ldots, y_{n-N}\right)$ satisfies (B.3), with $\left(y_{n}, \ldots, y_{n-N}\right)$ in $U$ and $y_{n+1}$ in $V$, if and only if

$$
y_{n+1}=H\left(y_{n}, \ldots, y_{n-N}\right)
$$

The resulting "delayed" difference equation can now be cast in the form (B.1) by considering the variable $x_{n}=\left(y_{n}, \ldots, y_{n-N}\right)$, and the map $G$ that associates to every such $x_{n}$ in $U$ the vector $x_{n+1}=\left(y_{n+1}, \ldots, y_{n-N+1}\right)$, with $y_{n+1}$ given by (B.4). One may note that $\bar{x}=(\bar{y}, \ldots, \bar{y})$ is a fixed point of $G$, and that the Jacobian matrix of $G$ at $\bar{x}$ has the form

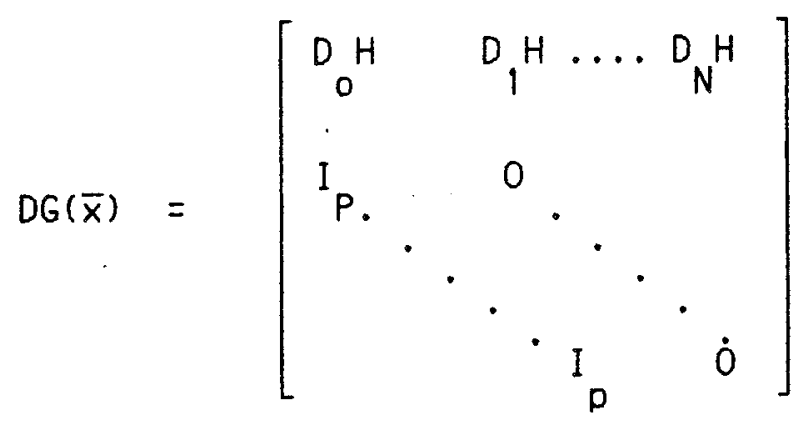

in which $D_{k} H, k=0, \ldots, N$, stands for the matrix of partial derivatives of $H$ with respect to the components $y_{n-k}$, evaluated at $(\bar{y}, \ldots, \bar{y})$, and $I_{p}$ is the $p$-dimensional identity matrix. It is easy to see that $D G(\bar{x})$ is invertible if and only if $\mathrm{D}_{N} \mathrm{H}$ is itself invertible. In that case, by the Inverse function Theorem A.2.1, one can choose $U$ small enough to ensure that $G$ is actually a $C^{r}$ diffeomorphism onto the open set $G(U)$. 


\section{B.1. Stability}

Consider the difference equation $(B .1)$ in which $G$ maps the open subset $U$ of $R^{m}$ into $R^{m}$. For any $x$ in $U$, one constructs the trajectory (or the orbit) of $x$ by using repeatedly (B.1) for $n \geq 0: x_{0}=x, x_{1}=G(x), \ldots, x_{n}=G^{n}(x)$, in which $G^{n}(x)$ is the $n$-th iterate of $x$ and is defined recursively through $G^{n}(x)=G\left(G^{n-1}(x)\right)$. Of course, it may occur that for some $n, G^{n}(x)$ no longer belongs to $U$; in that case, the orbit leaves the domain of definition of $G$ and is defined for only finitely many $n \geq 0$. If $x$ belongs to an invariant set $K$, i.e. a subset of $U$ such that $G(K)$ is contained in $K$, then the orbit of $x$ is defined for all $n \geq 0$. This will be the case for all , in particular, if $U=R^{m}$.

A fixed point $\bar{x}$ of $G$ is stable if for every open subset $V$ of $U$ containing $\bar{x}$, there is an open subset $V_{1}$ of $V$, with $\bar{x}$ also in $V_{1}$, such that for every $x$ in $V_{1}$, the iterates $G^{n}(x)$ are we 11 defined and belong to $V$ for all $n \geq 0$. If in addition, $V$ can be chosen so that the distance of $G^{n}(x)$ to $\bar{x}$ tends to zero as $n \rightarrow+\infty$ for each $x$ in $v_{1}$, then $\bar{x}$ is asymptotically stable. A fixed point is unstable if it is not stable.

Remark B.1.1. The foregoing notions of (asymptotic) stability apply not only to fixed points, but also to any closed set $K$, without any change.

Remark B.1.2. The analysis of the present appendix applies immediately to the study of what happens near a periodic orbit. Suppose that $G^{n}(\bar{y})=\bar{y}$ for some $\bar{y}$ in $U$ and $n>1$, with $G^{j}(\bar{y}) \neq y$ for $j=1, \ldots, n-1$. Then $\bar{y}$ is a periodic point, of period $n$, and the corresponding periodic orbit is defined as $\bar{y}$, $G(\bar{y}), \ldots, G^{n-1}(\bar{y})$. The map $F=G^{n}$ is defined at least on a sma 11 open 
neighbourhood $V$ of $\bar{y}$. Stability or unstability of the periodic orbit in the dynamics generated by $G$ is equivalent to stability or unstability of the fixed point $\bar{y}$ in the dynamics generated by $F$.

\section{B.2. Change of variables}

There is nothing intrinsic in the formulation embodied in (B.1). Indeed, one may always make a change of variables of the form $y=h(x)$, in which $y$ is a vector of $R^{m}$ and $h$ an homeomorphism from $R^{m}$ onto itself. The difference equation obtained after the change of variable is $y_{n+1}=f\left(y_{n}\right)$, in which $F=h \circ G \circ h^{-1}$ is a map from $h(U)$ into $R^{m}$ (actually, $h$ need be defined only on an open set containing both $U$ and $G(U))$. The two maps $F$ and $G$ are then said to be topologically conjugate. If $h$ is a $C^{r}$ diffeomorphism, they are $C^{r}$ conjugate. Clearly, two difference equations (or two maps) deduced from each other through conjugation generate the same trajectories (up to the change of variable); they must therefore be viewed as equivalent.

Such an equivalence relation may be only local. Let $\bar{x}$ be a fixed point of $G$, and consider another map $y \rightarrow F(y)$, with fixed point $\bar{y}$. Then $G$ is locally topologically (or $C^{r}$ ) conjugate to $F$ if there is an open neighbourhood $V$ of $\bar{x}$, and an open neighbourhood $W$ of $\bar{y}$, such that the restriction of $G$ to $V$ is topologically (or $C^{r}$ ) conjugate to the restriction of $F$ to $W$. In that case, only the orbits generated by $G$ that are sufficiently near $\bar{x}$, are the same, up to the change of variable, to those of $F$ that are near $\bar{y}$.

This notion of equivalence between maps or difference equations is important, since it allows one transforming a given difference equation, which may be difficult to analyse, and bringing it through an appropriate change of 
variables into a more tractable form, where the qualitative features of the trajectories may be more clearly seen. This equivalence notion leads also, quite naturally, to the idea of structural stability.

If the equation ( $B .1)$ is meant to describe the evolution of some physical or social phenomenon, one cannot be exactly sure about all the details of the specification. To get reliable results, one should accordingly work with maps $G$ that yield a qualitative picture that remains unaltered, up to a change of variable, when they are slightly perturbated. To be precise, assume that $G: U \rightarrow R^{m}$ is $C^{r}$, and let us endow the space of all $C^{r}$ maps $F: U \rightarrow R^{m}$ with the socalled $c^{r}$ - topology, i.e. the topology of uniform convergence, on compacta, of the values of the fonctions and of its derivatives up to the order $r$. The map $G$ is then $C^{r}$ - structurally stable if there is a neighbourhood $V$ of $G$, in the $c^{r}$ topology, such that all $c^{r}$ perturbations $F$ of $G$ that lie in $V$, are conjugate to.G. The qualitative features of the trajectories generated by a structurally stable map are unchanged after small perturbations.

Here again, structural stability may be required only locally. The $c^{r}$ $\operatorname{map} G: U \rightarrow R^{m}$ is $C^{r}$ locally structurally stable (near a fixed point $\bar{x}$ ) if there exists an open neighbourhood $V$ of $\bar{x}$ such that the restriction of $G$ to $V$ is $c^{r}$ - structurally stable.

The foregoing notion of structural stability involves arbitrary small perturbations of the map $G$ under consideration. If particular features of the phenomenon to be represented impose restrictions (e.g. symmetry) on the class of maps to be considered, then of course, perturbations and structural stability should be defined relatively to this particular class of maps. 
Remark B.2.1. Difference equations may be defined more generally on manifolds (a subset $M$ of $R^{p}$ is a $C^{r}$ manifold of dimension $m \leqq p$, if each e lement of $M$ has a neighbourhood $V$ for which there is a $C^{r}$ diffeomorphism that maps $V$ onto $R^{m}$ ). We shall need only exceptionally to consider explicitly such a more general formulation. But it may be worth noting at this stage that the study of the qualitative properties of trajectories near a fixed point in such a case, may be reduced to the analysis of the text, through an appropriate change of variable. Specifically, let $y_{n+1}=F\left(y_{n}\right)$ be the difference equation to be studied, where $F$ is a map from an open subset of a $C^{r}$ m-dimensional manifold $M$ into $M$. If $\bar{y}$ is a fixed point of $F$, there is a neighbourhood $V$ of $\bar{y}$, and a $C^{r}$ diffeomorphism $h$ mapping $V$ onto $R^{m}$. If $F$ is continuous, there is an open neighbourhood $W$ of $\bar{y}$ such that both $W$ and $F(W)$ are subsets of $V$. Making the change of variable $x=h(y)$, with $y$ in $W$, shows that the restriction of $F$ to $W$ is $C^{r}$ conjugate to the map $G: U+R^{m}$, where $U=h(W)$ and $G=h \circ F \circ h^{-1}$.

\section{B.3. Linear difference equations}

Linear difference equations of the form

$$
x_{n+1}=A x_{n}
$$

in which $x$ is a point of $R^{m}$ and $A$ is an m-dimensional square matrix, are important to study on their own right. In addition, as mentioned earlier, the trajectories associated to a nonlinear difference equation like (B.1) that are near enough a fixed point $\bar{x}$, generally behave qualitatively as those associated to the linearized version (B.2).

An obvious fixed point of (B.5) is the origin $x=0$, and an important issue is to determine its stability. The issue is not difficult to analyze, 
since the orbit of a point $x_{0}$ of $R^{m}$ through the repeated action of (B.5) is given by $x_{n}=A^{n} x_{0}$, in which $A^{n}$ is the $n$-th power of $A$, for all $n \geq 0$. $A^{n}$ may not be easy to compute, however, so we appeal to Theorem A.1.4, to assert the existence of a linear change of variable $x=P y$, in which $P$ is invertible, that transforms $(B .5)$ in

$$
y_{n+1}=B_{n}
$$

where $B=P^{-1} A P$ is the real canonical form of $A$. The matrix $B$ is much easier to work with, since it is block diagona $1, i . e, B=\operatorname{diag}\left(B_{1}, \ldots, B_{p}\right)$, in which each $B_{i}$ is the matrix corresponding to a generalized real eigenspace $F_{i}$. associated to an eigenvalue (and its conjugate if it is nonreal), equiped with a canonical basis. Since each eigenspace is invariant under the action of (B.6), one can solve separately each equation $\varepsilon_{n+1}=B_{i} \varepsilon_{n}$, where $E$ is a vector in the eigenspace $F_{i}^{*}$, which leads to $\varepsilon_{n}=B_{i}^{n} E_{0}$. Complete solutions of (B.6) are obtained by piecing together the solutions associated to each eigenspace, and one gets the solution of the original problem by making the change of variable $x=P y$.

\section{Distinct eigenvalues}

When $A$ has distinct eigenvalues, the form of $B$ was given in Theorem A.1.2. Restricting attention to the real eigenspace $F(\lambda)$ corresponding to the real eigenvalue $\lambda$ leads to the onedimensional difference equation $\xi_{n+1}=\lambda E_{n}$, or equivalently to the geometric sequence $\xi_{n}=\lambda^{n} \varepsilon_{0}$, in which the real number $\varepsilon_{n}$ is the coordinate of $y_{n}$ in $F(\lambda)$. Thus if $|\lambda|>1$, the sequence $\left|\varepsilon_{n}\right|$ diverges monotonically to $+\infty$ for every nonzero initial value $\varepsilon_{0}$. If $|\lambda|<1$, 
the sequence converges to 0 , all points going to 0 on first iterate when $\lambda=0$ (the map $E \rightarrow \lambda E$ is then noninvertible). If $\lambda=1$, then all points of $F(\lambda)$ are fixed points. If $\lambda=-1$, all points of the eigenspace that differ from 0 are periodic, with period 2 . The restriction of (B.6) to $F(\lambda)$ is orientation preserving when $\lambda>0$, and orientation reversing when $\lambda<0$.

Considering on the other hand the real eigenspace $F(\mu)$ corresponding to the nonreal eigenvalue $\mu=a+i b$, with $b>0$, leads to the twodimensional difference equation

$$
\xi_{n+1}=D E_{n}, \text { with } D=\left[\begin{array}{cc}
a & -b \\
b & a
\end{array}\right]
$$

or equivalently $\varepsilon_{n}=D^{n} \varepsilon_{0}$, where the twodimensional vector $\xi_{n}$ represents the coordinates of $y_{n}$ in the canonical basis of $F(\mu)$. Setting $a=0 \cos \theta$, $b=\rho \sin \theta$ with $\varrho=|\mu| \neq 0$ and $0<\theta<\pi$, one verifies easily that the action of $D$ in $R^{2}$ is a rotation of angle $\theta$ around the origin, followed by an homothecy, the center of which is the origin, with ratio e, see Fig. B.1. Thus

$$
D^{n}=e^{n}\left[\begin{array}{cc}
\cos n \theta & -\sin n \theta \\
\sin n \theta & \cos n \theta
\end{array}\right]
$$

If $|\mu|>1$, the sequence $\left|\varepsilon_{n}\right|$ diverges monotonically to $+\infty$ whenever $\varepsilon_{0} \neq 0$. It converges monotonically to 0 when $|\mu|<1$ (the map $E \rightarrow D E$ is then a contraction). If $|\mu|=1, D$ is a rotation of angle $\theta$ around the origin. Thus $\left|y_{n}\right|=\left|y_{0}\right|$ for all $n$, and every circle with center the origin is left invariant by $D$. If $\theta$ is of the form $2 \pi p / q$, where $p$ and $q$ are positive integers that are relatively prime, every point $y \neq 0$ of the plane is periodic, with period $q$, since then $D^{q} y=y$. If $\theta / 2 \pi$ is irrational, no point of the 
plane is periodic; the trajectory generated by $y_{0} \neq 0$ is in fact dense in the circle centered at the origin of radius $\left|y_{0}\right|$. In all cases, the restriction of (B.6) to the real eigenspace $F(\mu)$ is orientation preserving.

\section{Fig. B.1}

An important remark is in order here. The foregoing contracting (or expanding) properties of the application $\xi \rightarrow D E$ are valid when one uses the Euclidean norm $|E|$ of $R^{2}$. These properties do not hold any longer, in general, when the application is expressed in another coordinate system, e.g. through a change of variable $x=Q E$, where $Q$ is an invertible 2-matrix, if one sticks to the Euclidean norm $|x|$. Clearly, however, the map $x \rightarrow Q D Q^{-1} x$ has the same contracting or expanding properties as $E \rightarrow D E$, if one chooses the norm ||$x||=10^{-1} \times 1$.

\section{Muitiple eigenvalues}

When $A$ has multiple eigenvalues, the real canonical form $B$ is more complicated, and a little more care is needed. Consider first the case of a multiple real eigenvalue $\lambda$. As stated in Theorem A.2.4, the matrix $\wedge$ associated to the corresponding real generalized eigenspace $F^{\star}(\lambda)$ in the real canonical form, is block diagonal and composed of blocks of the form

$$
L=\left[\begin{array}{llllll}
\lambda & & & & \\
1 & \cdot & & & \\
& \cdot & \cdot & & \\
& & \cdot & \cdot & \\
& & & 1 & \lambda
\end{array}\right]
$$

Consider one of these matrices $L$, and let $p \geq 2$ be its dimension (the case 


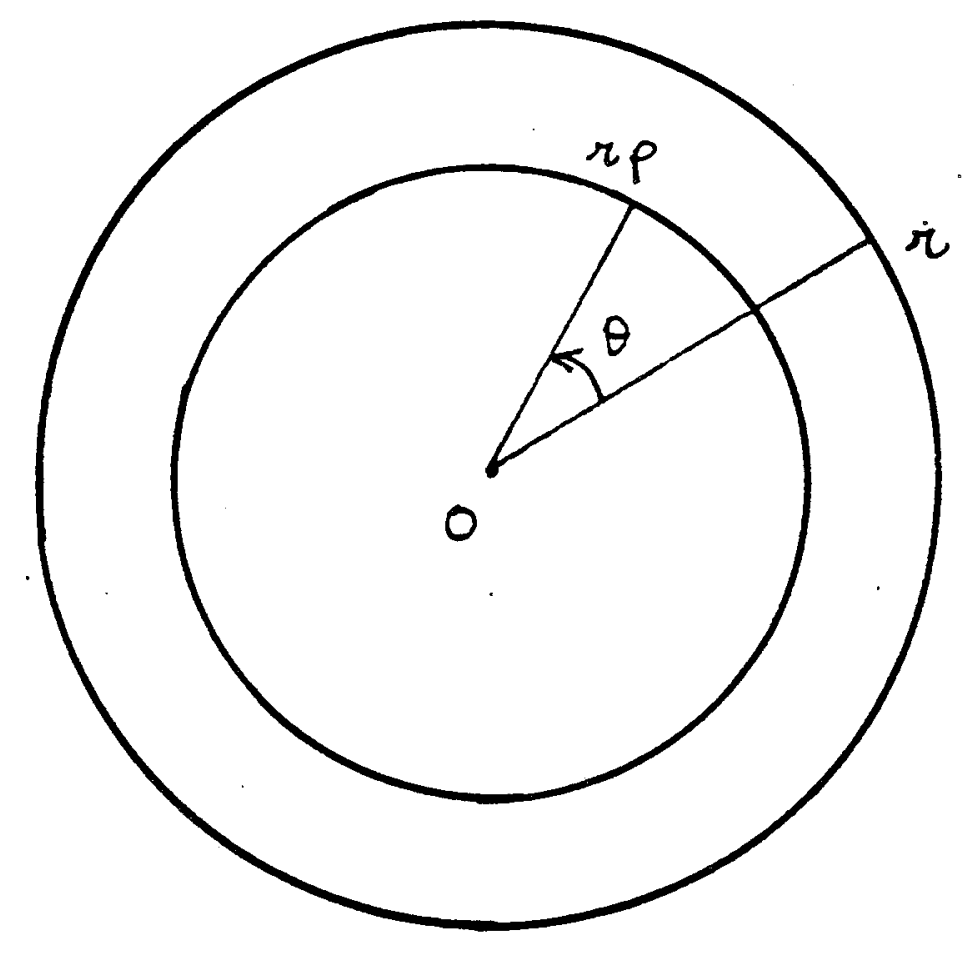

Fig. B.1 
$p=1$ was already analyzed for distinct eigenvalues). Restricting attention to the subspace spanned by the elements of the canonical basis associated to $L$, one is led to study the equation $\varepsilon_{n+1}=L \varepsilon_{n}$, or $\varepsilon_{n}=L^{n} \varepsilon_{0}$ for $n \geqslant 0$, where the p-dimensional vector $\varepsilon_{n}$ represents the coordinates of $y_{n}$ in the subspace under consideration.

To compute convenient ly $L^{n}$, remark that $L=S+N$, in which the diagonal matrix $S$ is $\lambda I_{p}$ and

$$
N=\left[\begin{array}{llllll}
0 & & & & \\
1 & \cdot & & & \\
& \cdot & \cdot & \\
& \cdot & \cdot & \\
& & \cdot & 1 & 0
\end{array}\right]
$$

The two matrices $S$ and $N$ commute, i.e. $S N=N S$, and thus

$$
L^{n}=\sum_{h=0}^{n}\left(\begin{array}{l}
h \\
n
\end{array}\right) \lambda^{n-h} N^{h},
$$

with the convention $N^{0}=I_{p}$. It is easily verified that

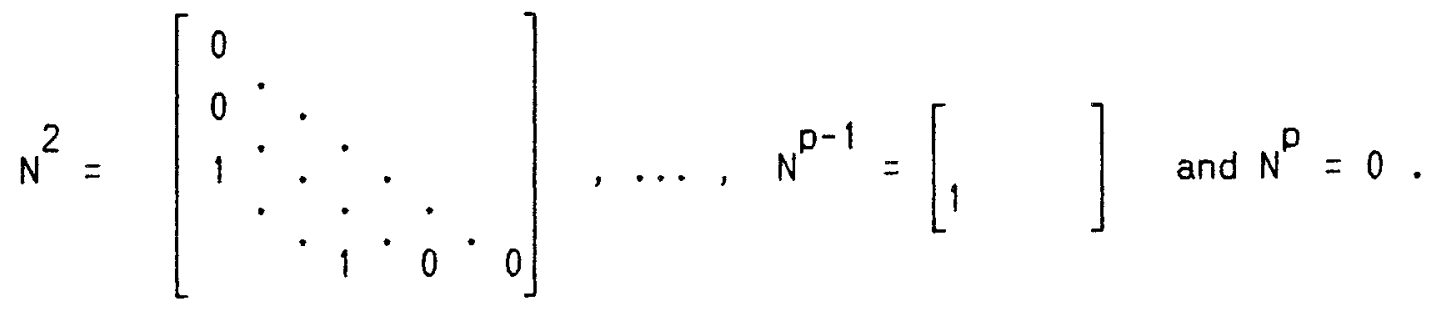

The components of $\xi_{n}$ are then given by the formulas

$$
\begin{aligned}
& \left(\varepsilon_{n}\right)=\lambda^{n}\left(\xi_{0}\right) \\
& \left(\varepsilon_{n 2}\right)=\lambda^{n}\left(\varepsilon_{0}\right)+\left(\begin{array}{l}
1 \\
n
\end{array}\right) \lambda^{n-1}\left(\xi_{0}\right)
\end{aligned}
$$




$$
\left(\varepsilon_{n}\right)=\lambda^{n}\left(\varepsilon_{0}\right)+\left(\begin{array}{l}
1 \\
n
\end{array}\right) \lambda^{n-1}\left(\varepsilon_{0}\right) p-1+\cdots+\left(\begin{array}{l}
s \\
n
\end{array}\right) \lambda^{n-s}\left(\varepsilon_{0}\right)
$$

with $s=\operatorname{Min}(n, p-1)$. It follows that $\left|\xi_{n}\right|+0$ when $|\lambda|<1$, and that $\left|\xi_{n}\right|++\infty$ when $\varepsilon_{0} \neq 0$ and $|\lambda|>1$, as $n$ goes to $+\infty$. The convergence (or divergence) need no be monotonic here, however. But one may show that there exists a norm $\|\xi\|$ such that the map $E \rightarrow L E$ is a contraction of modulus $k<1$ when $|\lambda|<1$ (such that the same property is true for the inverse mapping $\xi \rightarrow L^{-1} \xi$ when $\left.|\lambda|>1\right)$. If $|\lambda|=1$, the origin is unstable. One may verify that the restriction of $(B .6)$ to $F^{\star}(\lambda)$ is orientation preserving if $\lambda \geq 0$, or if $\lambda<0$ and the multiplicity of $\lambda$ is even, and that it is orientation reversing if $\lambda<0$ and the multiplicity of $\lambda$ is odd.

The case of a multiple nonreal eigenvalue $\mu=a+i b, b>0$, is handled by similar'methods. According to Theorem A.2.4, the matrix $\Delta$ associated in the real canonical form to the real generalized eigenspace $F^{*}(\mu)$, is block diagonal and composed of blocks of the form

$$
M=\left[\begin{array}{rrrrr}
D & & & & \\
I & \cdot & & \\
& \cdot & \cdot & \\
& & \cdot & \\
& & & 1 & 0
\end{array}\right] \text {, with } D=\left[\begin{array}{ll}
a & -b \\
b & a
\end{array}\right], I=\left[\begin{array}{ll}
1 & \\
& 1
\end{array}\right] \text {. }
$$

Consider one of these matrices $M$ and let $2 q$, with $q \geq 2$, be its dimension (again, the case $q=1$ was analysed for distinct eigenvalues). Restricting attention to the subspace spanned by the elements of the canonical basis corresponding to $M$, leads to the study of the equation $\xi_{n+1}=M \xi_{n}$, or of the sequence $\varepsilon_{n}=M^{n} \varepsilon_{0}, n \geq 0$, where $\varepsilon_{n}$ stands for the coordinates of $y_{n}$ in (B.6) in the subspace under consideration. 
One has here $M=S+N$, in which

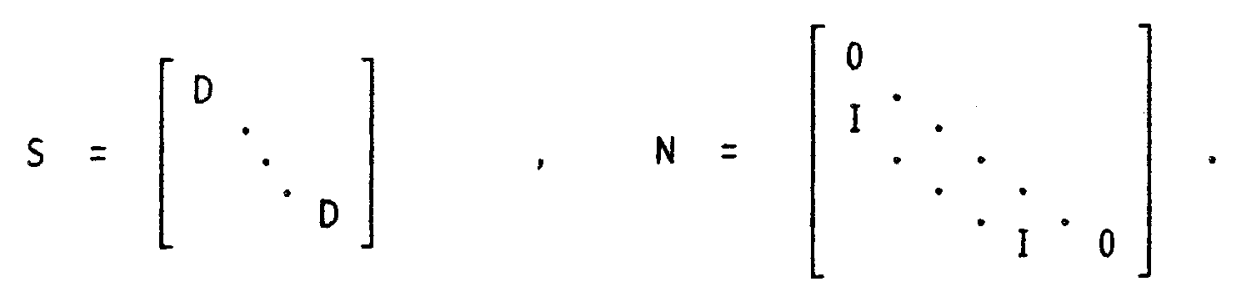

The matrices $S$ and $N$ commute, and thus

$$
M^{n}=\sum_{h=0}^{n}\left(\begin{array}{l}
h \\
n
\end{array}\right) D^{n-h} N^{h}
$$

The form of $D^{n-h}$ was given when considering distinct eigenvalues. If $a=e \cos \theta$ $b=\rho \sin \theta$ with $0=|\mu|$ and $0<\theta<\pi$, then

$$
D^{n-h}=0^{n-h} \quad\left[\begin{array}{cc}
\cos (n-h) \theta & -\sin (n-h) \theta \\
\sin (n-h) \theta & \cos (n-h) \theta
\end{array}\right]
$$

As for $N^{h}$, it is easily verified that

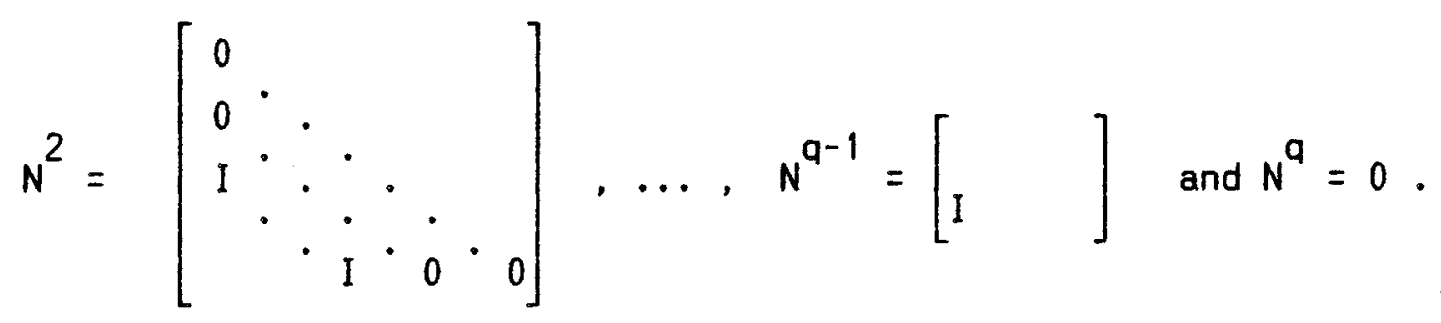

The components of $\varepsilon_{n}$ can then be easily computed from $\varepsilon_{n}=M^{n} \varepsilon_{0}$, in much the same way as for real eigenvalues. It will suffice here to remark that each component of $E_{n}$ is a linear combination of terms of the form

$$
\left(\begin{array}{l}
h \\
n
\end{array}\right) e^{n-h} \cos (n-h) \theta,\left(\begin{array}{l}
h \\
n
\end{array}\right) e^{n-h} \sin (n-h) \theta
$$


with $h=0, \ldots, \operatorname{Min}(n, q-1)$, the coefficients being determined by the initial vector $\varepsilon_{0}$. It follows here again that $\left|\varepsilon_{n}\right| \rightarrow 0$ when $\theta=|\mu|<1$, and $\left|\varepsilon_{n}\right|++\infty$ when $\varepsilon_{0} \neq 0$ and $|\mu|>1$, as $n$ diverges to $+\infty$. The convergence or divergence need not be monotonic. But there exists a norm ||$E||$ such that $\xi \rightarrow M E$ is a contraction of modulus $k<1$ when $|\mu|<1$ (the same being true of the inverse mapping $\varepsilon \rightarrow M^{-1} E$ if $\left.|\mu|>1\right)$. If $|\mu|=1$, the origin is unstable. In all cases, the restriction of $(B .6)$ to $F^{\star}(\mu)$ is orientation preserving.

This completes the analysis of the solutions of (B.6). One important feature of the solutions is the following one.

Theorem B.3.1. Every component of $y_{n}=B^{n} y_{0}, n>0$, is a linear combination of terms of the form

$$
\left(\begin{array}{l}
h \\
n
\end{array}\right) e^{n-h} \cos (n-h) \theta,\left(\begin{array}{l}
h \\
n
\end{array}\right) e^{n-h} \sin (n-h) \theta
$$

where $\lambda=e(\cos \theta+i \sin \theta)$ is an eigenvalue with multiplicity $k \geq 1$, and $h=0, \ldots, \operatorname{Min}(n, k-1)$ with weights determined by the initial vector $y_{0}$ (rea) eigenvalues correspond to $\theta=0, \pi)$. The solutions to $(B .5)$, being obtained through the linear transformation $x_{n}=P y_{n}$, have the same qualitative feature. 
The other important finding concerned the stability of the origin in $(B .6)$, and thus in (B.5). To state the results neatly, it is convenient to introduce the following notation. Let $\lambda_{1}, \ldots, \lambda_{r}$ be the distinct real eigenvalues of $A$, and $\mu_{1}, \bar{\mu}_{1}, \ldots, \mu_{s}, \bar{\mu}_{s}$ its distinct nonreal eigenvalues. The stable space $F^{s}$ of the origin is defined as the space spanned by all the vectors that lie in the real generalized eigenspaces $F_{h}^{*}(\lambda)$ or $F_{k}^{*}(\mu)$ such that $\left|\lambda_{h}\right|$ or $\left|\mu_{k}\right|<1$. The unstable space $F^{u}$ is the space spanned by the eigenspaces $F^{*}\left(\lambda_{h}\right)$ or $F^{*}\left(\mu_{k}\right)$ such that $\left|\lambda_{h}\right|$ or $\left|\mu_{k}\right|>1$. The center space $F^{c}$ is the space spanned by all the real generalized eigenspaces corresponding to an eigenvalue of modulus 1. Each of these spaces $F^{s}, F^{u}, F^{c}$ is of course invariant under (B.5). Moreover

Theorem B.3.2. There exist a norm $\|x\|$ in $R^{m}$ and two constants $0 \leqq k<1<k_{u}$ such that every solution $x_{n}=A^{n} x_{0}$ of (B.5) with $x_{0} \neq 0$ satisfies

1. $\left\|x_{n}\right\| \leq k_{s}^{n}\left\|x_{0}\right\|$ when $x_{0} \in F^{s}$, for a $11 n \geq 1$.

2. $\left\|x_{n}\right\|>k_{u}^{n}\left\|x_{0}\right\|$ when $x_{0} \in F^{u}$, for all $n \geq 1$.

3. The sequence $x_{n}$ does not converge to 0 when $x_{0} \in F^{c}$.

If a 11 eigenvalues of $A$ are such that $|\lambda|<1$, the origin is a sink ; if $|\lambda|<1$ for some eigenvalues and $|\lambda|>1$ for the others : a saddle point, and if $|\lambda|>1$ for all eigenvalues : a source.

\section{Structural stability}

It is clear that the linear map $x \rightarrow$ Ax cannot be structurally stable 
(even in the class of linear applications of $R^{m}$ ) if one of the eigenvalues (or more) has modulus one. For the trajectories lying in the center space $F^{C}$ can then be removed by a slight perturbation of the matrix $A$. Nor can it be structurally stable if one of the eigenvalues (or more) is 0 . One may expect, however, the linear map to be structuraliy stable if these two "borderline" cases are assumed away. This conjecture is confirmed by the following fact.

Theorem B.3.3. Assume that the m-dimensional matrix A has no eigenvalue of modulus equal to 0 or 1 . Then the linear map $x \rightarrow$ Ax is structurally stable in the class of all linear maps of $R^{m}$.

\section{B.4. Hyperbolic fixed points}

Let us go back to the nonlinear difference equation

$$
x_{n+1}=G\left(x_{n}\right)
$$

where the map $G: U \rightarrow R$ is $C^{r}$, and consider a fixed point $\bar{x}$ of $G$. A common procedure for studying the solutions of this equation near $\bar{x}$, is to linearize it and to analyse the solutions of the associated linear equation

$$
y_{n+1}=D G(\bar{x}) y_{n}
$$

in which $D G(\bar{x})$ is the Jacobian matrix of $G$ at $\bar{x}$. The following fact implies that the procedure is valid, provided that $D G(\bar{x})$ is invertible (has no zero eigenvalue), and that $\bar{x}$ is a hyperbolic fixed point, i.e. DG( $\bar{x})$ has no eigenvalue of modulus 1 .

Theorem B.4.9. Let $G$ be a $C^{r}$ map, $r \geq 1$, from the open subset $U$ of $R^{m}$ into $R^{m}$. Let $\bar{x}$ be a hyperbolic fixed point such that the Jacobian matrix $D G(\bar{x})$ is 
invertible. Then $G$ is locally topologically conjugate to the linear map $y+D G(\bar{x}) y$

It follows from the analysis of the previous section that under the conditions of the theorem, the fixed point $\bar{x}$ is asymptotically stable if all eigenvalues $\lambda$ of $D G(\bar{x})$ satisfy $|\lambda|<1$, and that it is unstable when one of them is such that $|\lambda|>1$. As a matter of fact, this conclusion is valid even when some eigenvalues of $D G(\bar{x})$ are 0 .

Corollary B.4.2. Let $G$ be a $C^{r}$ map, $r \geqslant 1$, from the open subset $U$ of $R^{m}$ into $R^{m}$, and $\bar{x}$ a hyperbolic fixed point. Then $\bar{x}$ is asymptotically stable if all eigenvalues $\lambda$ of $D G(\bar{x})$ satisfy $|\lambda|<1$, and unstable if $|\lambda|>1$ for some $\lambda$.

In view of the result concerning the structural stability of linear maps of the previous section (Theorem B.3.3), one should expect, under the assumptions of Theorem B.4.1, the map $G$ to be locally structurally stable. Indeed

Corollary B.4.3. Under the assumptions of Theorem B.4.1, G is locally structura lly stable.

\section{B.5. Invariant manifolds}

It is convenient, when studying the solutions of a linear difference equation $x_{n+1}=A x_{n}$, to split the analys is into three parts, by looking separately at what happens in each of the three invariant subspaces that were introduced in Theorem B.3.2 : The stable space $F^{s}$, the unstable space $F^{u}$ and 
the center space $F^{c}$ of the matrix $A$. It turns out that a similar decomposition is also possible in the case of a nonlinear difference equation $x_{n+1}=G\left(x_{n}\right)$, at least locally, i.e. near a fixed point $\bar{x}$. The role of the above spaces is then played by local invariant surfaces that are tangent to the stable, unstable and center spaces of the Jacobian matrix $D G(\bar{x})$, respectively. To be specific, assume that $G: U \rightarrow R^{m}$ is $C^{r}, r \geq 1$, and that $\bar{x}$ is a fixed point. Given a ne ighbourhood $V$ of $\bar{x}$, a local invariant manifold $W_{(\bar{x})}$ is a $C^{\prime}$ manifold (a continuousiy differentiable surface) passing through $\bar{x}$ and contained in $V$, such that $x \in W_{(\bar{x})}, x \in V$ and $G(x) \in V$ imply $G(x) \in W_{(\bar{x})}$. A local stable manifold $W_{(\bar{x})}^{S}$ is then a local invariant manifold that is tangent at $\bar{x}$ to (and has the same dimension as) the stable space $F^{s}$ of the Jacobian matrix $D G(\bar{x})$. Similarly, a local unstable (or center) manifold $W_{(\bar{x})}^{u}$ (or $W_{(\bar{x})}^{c}$ ) is a local invariant manifold that is tangent at $\bar{x}$ to (and has the same dimension as) the unstable (or center) space $F^{u}$ (or $F^{c}$ ) of $D G(\bar{x})$.

Theorem B.5.1. Let $\bar{x}$ be a fixed point of the $c^{r}$ map $G$ from the open subset $U$ of $R^{m}$ into $R^{m}(r \geqslant 1)$. Then there exists a neighbourhood $V$ of $\bar{x}$ such that 1. There is a unique local stable manifold $w_{(\bar{x})}^{s}$ in $V$. It is $c^{r}$.

2. If $D G(\bar{x})$ is invertible, there is a unique local unstable manifold $W_{(\bar{x})}^{u}$ in $V$ (it is actually the local stable manifold of the local inverse $G^{-1}$ ). It is $c^{r}$.

3. Suppose $r \geqslant 2$. There is a (generally nonunique) local center manifold $W_{(\bar{x})}^{c}$ in $V$. It is $c^{r-1}$, and locally attracting: if $\times$ and $G^{n}(x)$ are in $V$ for all $n \geqslant 1$, then the distance between $G^{n}(x)$ and $W^{c}(\bar{x})$ tends to 0 as $n \rightarrow+\infty$. 
Of course, in the foregoing statement, if the dimension of one of the spaces $F$ is 0 (if the space reduces to the origin), the corresponding local manifold vanishes. Fig. B.2 describes local stable and unstable manifolds in the case of a hyperbolic fixed point in $R^{2}$.

\section{Fig. B.2}

\section{Motion on the local stable and unstable manifolds}

Since the local stable and unstable manifolds are diffeomorphic, near $\bar{x}$, to the stable and unstable spaces $F^{s}$ and $F^{u}$ of the Jacobian matrix $D G(\bar{x})$, one should expect, by analogy to Theorem B.4.1, the restriction of $G$ to these manifolds to behave near $\bar{x}$ like the restriction of the linearized map $x \rightarrow D G(\bar{x}) \times$ to $F^{s}$ or $F^{u}$. Indeed,

Theorem B.5.2. Suppose that $G$ is as in Theorem B.5.1, and that $D G(\bar{x})$ is invertible. Let $B^{S}, B^{u}$ be the submatrices in the real canonical form of $D G(\bar{x})$, that correspond to the stable and unstable spaces $F^{s}$ and $F^{u}$, respectively. Then

1. The restriction of $G$ to the local stable (resp. unstable) manifold is locally topologically conjugate to the linear map $x \rightarrow B^{S} x, x \in F^{s}$ iresp. $\left.x \rightarrow B^{u} x, x \in F^{u}\right)$.

2. There is a norm $\|x\|$ of $R^{m}$, and two constants $k^{s}<1<k^{u}$ such that if $\delta>0$ is small enough
a. $\|G(x)-\bar{x}\| \leq k^{s}|| x-\bar{x} \|$ for $a \| 1 \times$ in $w_{(\bar{x})}^{s},\|x-\bar{x}\|<\delta$. 


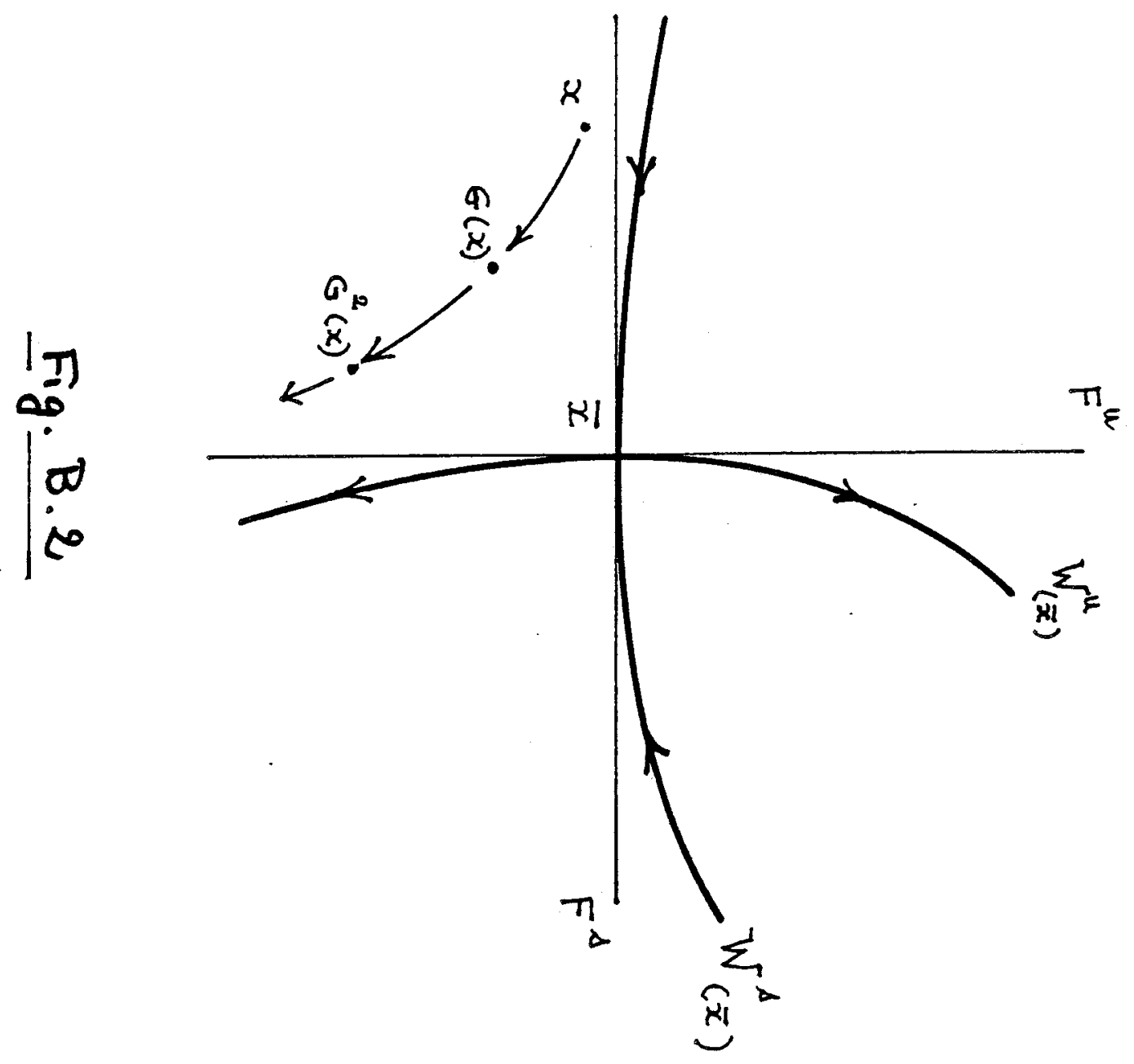


b. $\|G(x)-\bar{x}\| \geq k^{u}\|x-\bar{x}\|$ for a $\mid x$ in $w_{(\bar{x})}^{u},\|x-\bar{x}\|<\delta$.

\section{Motion on a local center manifold}

Part 3 of Theorem B.5.1 shows that any recurrent behaviour (e.g. cycles) near the fixed point, must occur in a local center manifold. An explicit representation of such a manifold, that takes into account possible nonlinearities, is therefore useful. It may be obtained through the following procedure (the reader will verify easily that the procedure applies to local stable and unstable manifolds as wel1).

To simplify notation, assume that the fixed point has been translated to the origin, i.e. $\bar{x}=0$, and that a linear change of variables has brought the Jacobian matrix $D G(\bar{x})$ in its real canonical form, which we write diag $\{C, B\rangle$, in which $C$ corresponds to the center space $F^{C}$, and $B$ to the direct sum of the stable and unstable spaces $F^{s}$ and $F^{u}$. In terms of the notation of Theorem $B .5 .2, B=\operatorname{diag}\left\langle B^{S}, B^{u}\right)$. In the new coordinate system $(\varepsilon, n)$, where $\varepsilon$ be longs to $F^{c}$ and $n$ to $F^{s}+F^{u}$, the difference equation $x_{n+1}=G\left(x_{n}\right)$ reads

$$
\begin{aligned}
& \xi_{n+1}=C \xi_{n}+f\left(\xi_{n}, n_{n}\right) \\
& n_{n+1}=B n_{n}+g\left(\xi_{n}, n_{n}\right)
\end{aligned}
$$

in which $f$ and $g$ are defined on an appropriate neighbourhood of the origin. By construction, the functions $f$ and $g$ are $c^{r}, r \geq 2$ and are equal to 0 , as well as their first partial derivatives, at $\varepsilon=0, \eta=0$.

Since a local center manifold $W_{(\bar{x})}^{c}$ is tangent at the origin to $F^{c}$ (the $\eta=0$ space), one can represent it as the graph of a $c^{r-1}$ function $n=r(\varepsilon)$, that is defined on a (small enough) open ball $\vee$ in $F^{c}$, centered at $\varepsilon=0$, 
such that $\gamma$ and its first partial derivatives vanish at $\varepsilon=0$, see Fig. 8.3. The function $\gamma$, near the origin, is given implicitly by expressing that $W_{(\bar{x})}^{C}$ is locally invariant, that is

$$
B \gamma(E)+g(\varepsilon, \gamma(E))=\gamma[C E+f(\varepsilon, \gamma(E))]
$$

whenever $E$ and $C E+f(E, \gamma(E)$ ) are in $V$ (which will be surely true if $\varepsilon$ is small enough). Given such a function $\gamma$, the restriction of the difference equation to the local center manifold is obtained by setting $\eta_{n}=\gamma\left(\varepsilon_{n}\right)$ and $n_{n+1}=r\left(\xi_{n+1}\right)$ in (B.9). Clearly, one obtains an equivalent difference equation by projecting it on $F^{c}$, i.e. by considering only the equation corresponding to the component $\varepsilon$

$$
\varepsilon_{n+1}=C E_{n}+f\left(E_{n}, \gamma\left(\varepsilon_{n}\right)\right) \equiv \Gamma\left(\varepsilon_{n}\right), \varepsilon_{n} \in V
$$

Trajectories in the local center manifold may then be analyzed by using this "reduced difference equation" in $F^{c}$, near $\xi=0$.

\section{Fiq. B.3}

\section{Equivalent local dynamics}

Up to now, we confined ourselves to the trajectories that lie in the local stable, center and unstable manffolds. The following fact shows that these trajectories contain all the information that is necessary to reconstruct the dynamics generated by $G$ in $R^{m}$, near the fixed point : as for the linear case, trajectories in $R^{m}$ may be considered as the "Cartesian product" of trajectories lying in each invariant manifold. Since the motion on the local stable and unstable manifolds is locally conjugate to the linear equation 
35

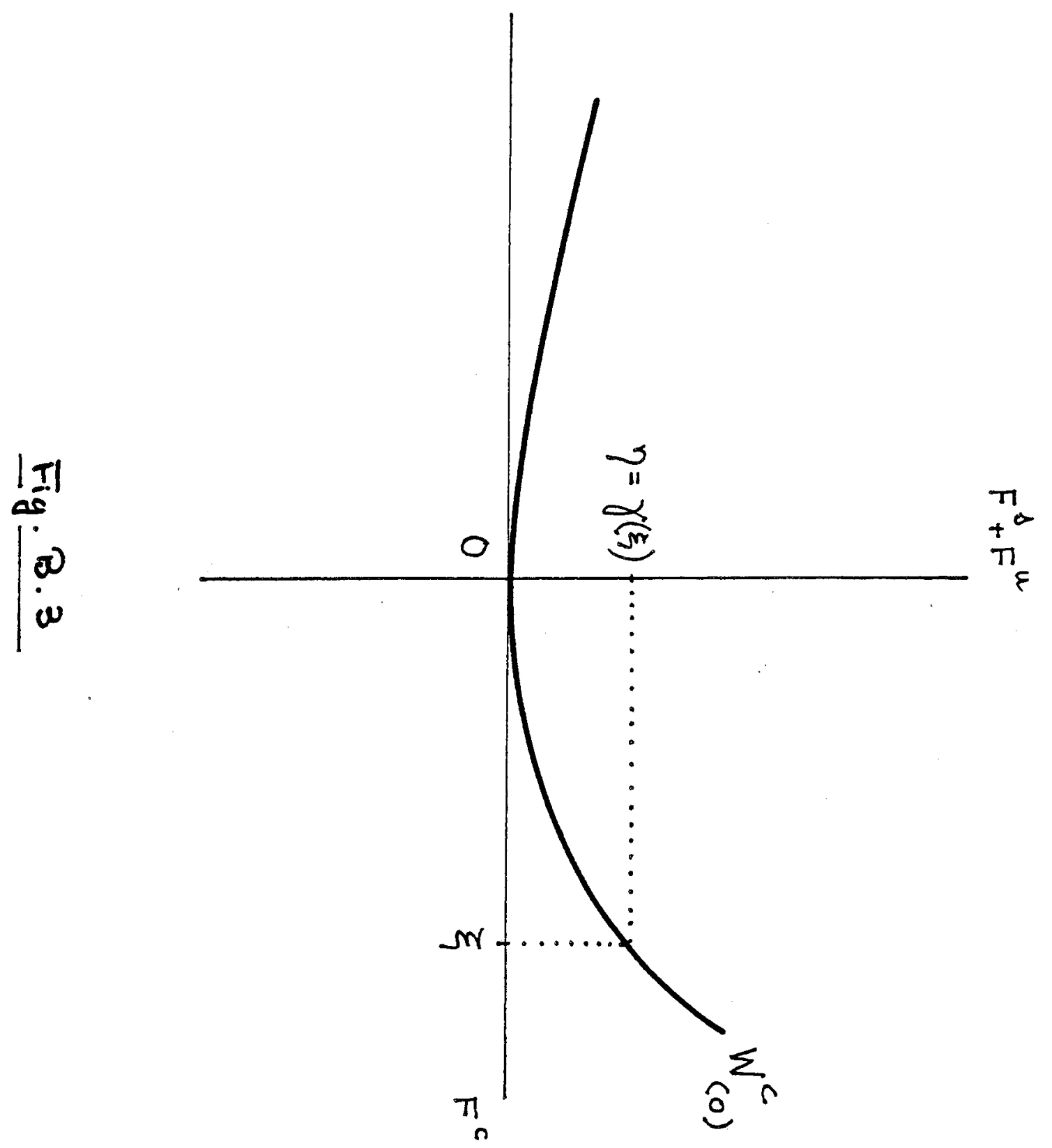


$\eta \rightarrow B n$, with $\eta$ in $F^{5}+F^{u}$, this result can be formulated as

Theorem B.5.3. Let $G$ be as in Theorem B.5.1, and assume that $D G(\bar{x})$ is

invertible. Then $G$ is locally topologically conjugate to the application

defined by

$$
\begin{aligned}
& \eta+B \eta, \eta \in F^{s}+F^{u} \text {, where } B=\operatorname{diag}\left\langle B^{s}, B^{u}\right\rangle \\
& \varepsilon \rightarrow C E+f(\varepsilon, \gamma(E))=\Gamma(\varepsilon), E \in F^{c} \text { small. }
\end{aligned}
$$

Of course, if the Jacobian matrix $D G(\bar{x})$ has no eigenvalue of modulus 1 , the foregoing result reduces to Theorem B.4.1.

Remark B.5.4. One cannot get, in general, an exact analytical expression of a solution $\gamma$ of (B. 10$)$, but one can approximate it by a Taylor series at $E=0$. Suppose that a $C^{\prime}$ function $T$ from $F^{c}$ into $F^{s}+F^{u}$ has been found such that, first, $T$ and its first partial derivatives vanish at $E=0$, and second

$$
T[C E+f(E, T(E))]-B T(E)-g(E, T(E))=O\left(|E|^{D}\right)
$$

as $\varepsilon+0$, for some $1<p<r$. Then $\gamma(\varepsilon)=T(E)+O\left(|x|^{D}\right)$ as $E+0$, see Carr (1981, Section 2.8). Here the notation $O\left(|E|^{p}\right)$ stands for a function $\alpha(E)$ such that there is a constant $k$ with $|\alpha(\xi)|<k|E|^{p}$ for $E$ small enough. In practice, $T(E)$ is chosen as a Taylor series of $\gamma$. Specifically, one expands $\gamma$, up to the desired order, as well as $f$ and $g$, as a Taylor series at $\varepsilon=0$ in (B.10), and one gets the unknown coefficients of the Taylor expansion of $\gamma$ by identification of the two members. This shows in particular that although the function $\gamma$ defining a local center manifold need not be unique, its partial derivatives a $\xi=0$ are nevertheless uniquely determined. Finally, replacing $\gamma$ by its Taylor approximation $T$ in (B.11) yields an approximate analytical 
expression of the reduced difference equation in the local center manifold.

Of course, the principle of such a Taylor approximation applies equally well to local stable and unstable manifolds.

\section{Notes on the literature}

The treatment of linear difference equations in section $B .3$ is a transposition of Hirsch and Smale (1974, chap. 6), who deal with differential equations. The material on hyperbolic fixed points of section 8.4 can be found in any book on dynamical systems, see e.g. Hartman (1964), Palis and de Melo (1982, chap. 2.4), Guckenheimer and Holmes (1983, chap. 1.4). Section B.5 on invariant manifolds is adapted from Lanford (1983), Iooss (1979, chap. V), Carr (1981, section 2.8), Guckenheimer and Hoimes (1983, chap. 1.4 and 3.2), Palis and de Melo (1982, chap. 2.6). Theorem B.5.3 follows from Palis and Takens (1977) : the analogue result for differential equations (vector fields) is stated there p. 341 (see also Guckenheimer and Holmes (1983, p. 130)), and the result for diffeomorphisms follows by a suspending technique, as $p .340$ (I owe this reference to $A$. Chenciner). 


\section{LOCAL BIFURCATIONS}

As we have seen in Section B.3, linear difference equations can produce cyclical trajectories when some eigenvalues have modulus 1 , but such cycles are removed by a small change of the matrix defining the system. By contrast, nonlinear maps may generate cyclical behaviour that is preserved under small perturbations, i.e. that is structurally stable. A most powerful tool to analyse the occurrence of cyclical fluctuations, in many cases the only one presently available, is provided by the theory of bifurcations.

\section{C.1. Introduction}

Consider a family of difference equations indexed by a real parameter $\alpha$, say $x_{n+1}=G_{\alpha}\left(x_{n}\right)$, where each $G_{\alpha}$ is a map from an open set $U$ of $R^{m}$ into $R^{m}, \alpha$ is in an open interval I containing 0 , and where the dependence on $(\alpha, x)$ is $c^{r}, r \geq 1$. In many cases, one requires also that $G_{\alpha}(0)=0$, for all admissible $\alpha$ : this simply means that there is a family of fixed points that have been translated to the origin, see Remark C.1.1. The family $G$ undergoes a bifurcation, say at $\alpha=0$, if the qualitative features of the orbits of $G$ change when $\alpha$ moves from negative to positive values. The bifurcation is local (at the origin) if the change of the orbit structure can be observed in an arbitrarily small (but independent of $\alpha$ ) neighbourhood of $x=0$; the bifurcation is global otherwise. We focus here on local bifurcations. A brief introduction to global bifurcations, which are far more difficult to understand, will be given in Appendix D.

If a local bifurcation occurs, then in view of Theorem B.4.2, there must be an eigenvalue $\lambda_{\alpha}$ of the Jacobian matrix $D G_{\alpha}(0)$ that either goes through 0 
$\left(\lambda_{\alpha}=0\right)$, or crosses the unit circle in the complex plane $\left(\left|\lambda_{\alpha}\right|=1\right)$, at $\alpha=0$. The case $\lambda_{\alpha}=0$ is uninteresting, however, since it may change locally a map that is orientation reversing to an orientation preserving one, but it cannot generate cycles near the origin (think of the one dimensional difference equation $x_{n+1}=\alpha x_{n}+0\left(\left|x_{n}^{2}\right|\right)$ for $\alpha, x$ smal1). We shall assume accordingly that the Jacobian matrix $D G(0)$ is invertible for each admissible $\alpha$, and focus on the case where only one eigenvalue $\lambda_{\alpha}$ (and of course its conjugate $\bar{\lambda}_{\alpha}$ when it is nonreal) having multiplicity one, crosses the unit circle at $\alpha=0$ : this is called a codimension one local bifurcation (1). There are thus three cases: if $\lambda_{0}=+1$, one gets a saddle node (or fold) bifurcation ; if $\lambda_{0}=-1$, a flip bifurcation, and if $\lambda_{0}$ is nonreal, a Hopf bifurcation.

It turns out that although the dimension of the ambient space $R^{m}$ may be large, all the recurrent behaviour associated with a local bifurcation occurs necessarily in an invariant surface (manifold) that has a dimension equal to the number of eigenvalues that cross simultaneousiy the unit circle at $\alpha=0$. The principle of such a drastic reduction of the dimension of the problem will be shown to be a consequence of the theory of local center manifolds in section C.5. This remarkable result enables us to study the saddle node and the flip bifurcations directly for difference equations on a onedimensional curve, or equivalently on the real line. On the other hand, a Hopf bifurcation may be analysed directly in $R^{2}$, since then the (nonreal) eigenvalue $\lambda_{\alpha}$ and its conjugate $\bar{\lambda}_{\alpha}$ have modulus 1 at the point of bifurcation $\alpha=0$.

Remark C.1.1. We describe now a frequent iy encountered situation, and show how it can generate the formulation described in the text. Assume, as in the 
introduction of Appendix B, that the successive states $y$ of a given physical or social system must satisfy

$$
F\left(y_{n+1}, y_{n}, \ldots, y_{n-N}, \alpha\right)=0
$$

where $y$ is a vector of $R^{p}$, $\alpha$ is a real number indexing some characteristics of the system, and $F$ is a $C^{r}$ map from an open set of $R^{p(N+2)+1}$ into $R^{p}$. Let $\bar{y}$ be a stationary state for $\alpha=0$, i.e. a vector of $R^{p}$ such that $F(\bar{y}, \ldots, \bar{y}, 0)=0$, and assume that the Jacobian matrix of the map $y+F(y, \bar{y}, \ldots, \bar{y}, 0)$ is invertible at $y=\bar{y}$. From the Implicit Function Theorem, there are open neighbourhoods $V$ of $(\bar{y}, \ldots, \bar{y}, 0)$ in $R^{p(N+1)+1}$, $W$ of $y$ in $R^{p}$, and a unique $C^{r}$ map $H: V \rightarrow W$ such that $\left(y_{n+1}, \ldots, y_{n-N}, \alpha\right)$ satisfies $(C .1)$, with $\left(y_{n}, \ldots, y_{n-N}, \alpha\right)$ in $v$ and $y_{n+1}$ in $W$, if and only if

$$
y_{n+1}=H\left(y_{n}, \ldots, y_{n-N}, \alpha\right) \text {. }
$$

To get the formulation of the text, one considers the variable $x_{n}=\left(y_{n}, \ldots, y_{n-N}\right)$, and the map $G$ that associates to every $\left(x_{n}, \alpha\right)$ in $V$ the vector $x_{n+1}=\left(y_{n+1}, y_{n}, \ldots, y_{n-N+1}\right)$ with $y$ given by (C.2). One may restrict $V$ to be the product of an open neighbourhood $U$ of $(\bar{y}, \ldots, \bar{y})$ in $R^{p(N+1)}$, and of an open interval I containing $O$ in $R$. Then $G(x, \alpha)$ is defined on $U \times I$; in other words, each map $G_{\alpha}=G(, \alpha)$ is well defined for each admissible $\alpha$, on the open set $U$, which is independent of the parameter $\alpha$.

We remark that $\bar{x}=(\bar{y}, \ldots, \bar{y})$ is a fixed point for $\alpha=0$, i.e. $G(\bar{x}, 0)-\bar{x}=0$. This fixed point may of course be translated to the origin through a linear change of variable. In fact, as noted in the text, one often 
requires that $G(0, \alpha)=G_{\alpha}(0)=0$ for all admissible $\alpha$. We describe now a procedure that leads to such a formulation.

Assume that the Jacobian matrix $D_{x} G(\bar{x}, 0)$ has no eigenvalue equal to 1 . Then, again from the Implicit Function Theorem, the fixed point is bound to persist for small values of $\alpha$. That is, the equation

$$
x-G(x, \alpha)=0
$$

can be solved uniquely in $x$ near $(\bar{x}, 0)$. Specifically, there are open neighbourhoods of $\alpha=0$ in $R$ and of $\bar{x}$ in $R^{p(N+1)}$ - which we may take, since we are interested in local bifurcations, to be $I$ and $U$ respectively - and a $C^{r}$ map from I to $U$, i.e. $\alpha+\bar{x}_{\alpha}$, such that (C.3) is satisfied on $U \times I$ if and only if $x=\bar{x}_{\alpha}$. Translating $\bar{x}_{\alpha}$ to the origin of $R^{p(N+1)}$ is achieved by making the change of variable $z=x-\bar{x}$, for each admissible $\alpha$, which yields the equivalent family $G^{*}(z, \alpha)=G\left(z+\bar{x}_{\alpha}, \alpha\right)-\bar{x}_{\alpha}$. One has then indeed $G^{\star}(0, \alpha)=0$ for all $\alpha$. By continuity of the map $\alpha \rightarrow \bar{x}_{\alpha}$, we are sure that each map $G_{\alpha}^{*}=G^{*}(\ldots, \alpha)$ is well defined on a small open ball $U^{*}$ centered at 0 in $R^{p(N+1)}$, for $|\alpha|<\delta$, if $\delta$ is small enough. Restricting attention to the family $G^{*}: U^{*} \rightarrow R^{p(N+1)},|\alpha|<\delta$, yields the desired formulation.

Remark C.1.2. It should be emphasised that the results of this appendix apply also to the study of the local bifurcations of a family of difference equations, near a periodic orbit. Consider a family of difference equations in $R^{m}$ given by the maps $F_{\alpha}$, each being defined on a given open set, and suppose that the origin $x=0$ is a fixed point of the $n$-th iterate of $F_{\alpha}$, i.e. $F_{\alpha}^{n}(0)=0$ for some $n>1$, with $F_{\alpha}^{j}(0) \neq 0$ for $j=1, \ldots, n-1$, for all 
admissible $\alpha$. Then $x=0$ is a periodic point, with period $n$, the

corresponding periodic orbit being $0, F_{\alpha}(0), F_{\alpha}^{2}(0), \ldots, F_{\alpha}^{n-1}(0)$, for each $\alpha$ (again this simply means that there is a family of periodic orbits, and that one of the periodic points on the orbits has been translated to the origin). All the results of this appendix can then be transposed to the case at hand by considering the family $G_{\alpha}=F_{\alpha}^{n}$.

\section{C.2. The saddle node bifurcation}

We look first at the case of a one-parameter family of difference equations on the real line

$$
x_{n+1}=G\left(x_{n}, \alpha\right) \equiv G\left(x_{n}\right)
$$

defined by the $C^{r} \operatorname{map} G: U \times I \rightarrow R$, where $U$ and $I$ are open intervals of the real line containing 0 . In that case, for each value of the parameter $\alpha$, the trajectories generated by the map $G_{\alpha}$ are easily visualised with the help of its graph, in the plane $\left(x_{n}, x_{n+1}\right)$, as shown in Fig. C.1. A fixed point $\bar{x}$ is then described as a point of intersection of the graph of $G_{\alpha}$ with the $45^{\circ} 1$ ine, and the trajectory associated with an initial condition $x_{0}$ is generated by following the arrows as in the Figure. A fixed point $\bar{x}$ of $G_{\alpha}$ is then asymptotically stable if $\left|\frac{\partial G}{\partial x}(\bar{x}, \alpha)\right|<1$, unstable if $\left|\frac{\partial G}{\partial x}(\bar{x}, \alpha)\right|>1$.

\section{Fiq. C.1}

Assume that the origin is a fixed point for $\alpha=0$, i.e. $G(0,0)=0$, and that $\frac{\partial G}{\partial x}(0,0)=+1$. A saddle node bifurcation will obtain if we suppose 


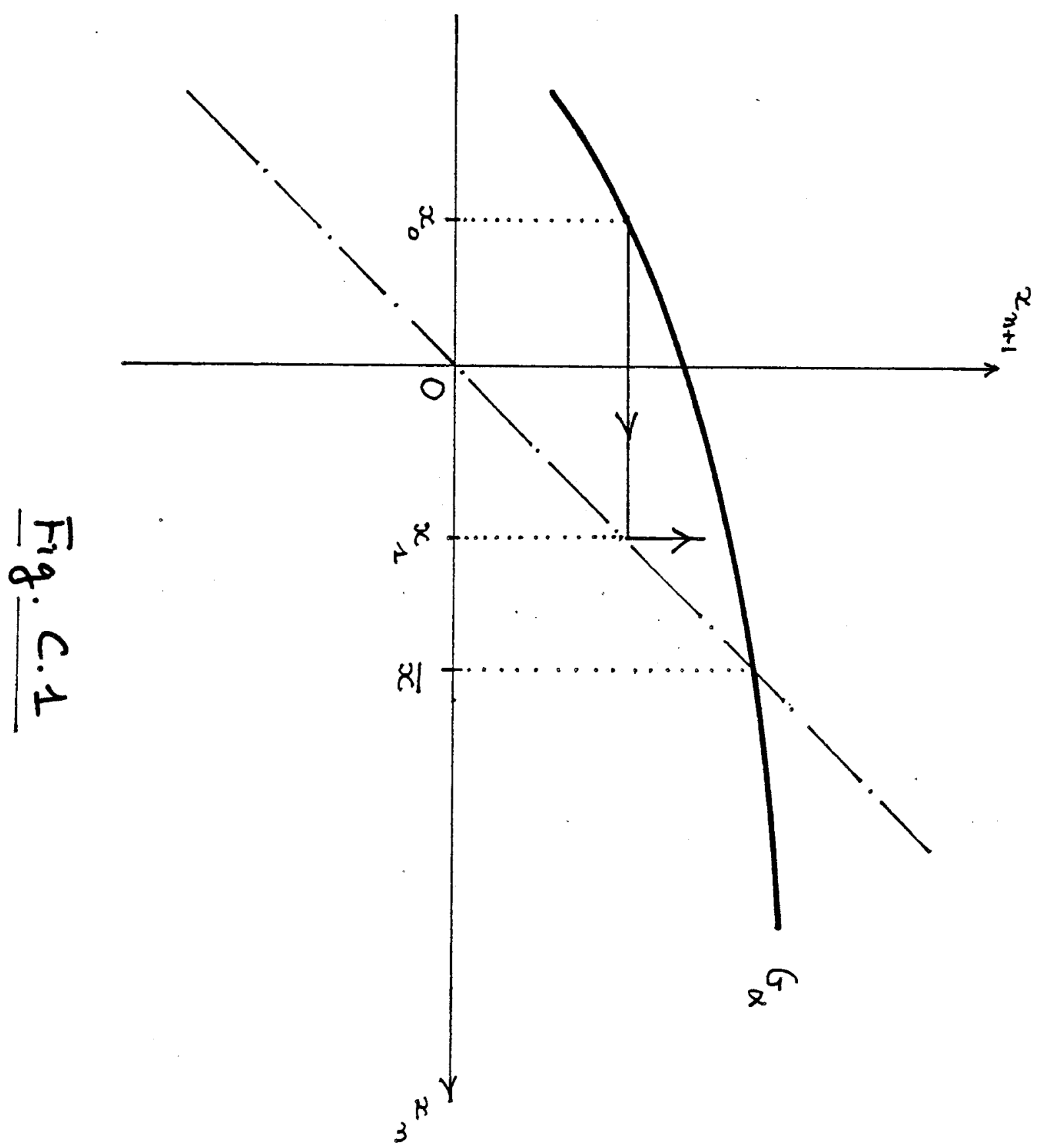


the "generic" conditions $\frac{\partial G}{\partial \alpha}(0,0) \neq 0$ and $\frac{\partial^{2} G}{\partial x^{2}}(0,0) \neq 0$ (we need accordingly $r \geq 2)$

Fig. C.2.a pictures what happens to the graphs of the maps $G_{\alpha}$ for $\alpha$ and $x$ sma 17, in the case where $\frac{\partial G}{\partial \alpha}(0,0)>0$ and $\frac{\partial^{2} G}{\partial x^{2}}(0,0)>0$. If $\alpha<0, G$ has two fixed points near 0 . The one on the left is asymptotically stable, the other is unstable. For $\alpha=0$, the origin is stable from the left, unstable from the right. If $\alpha>0, G_{\alpha}$ has no fixed point near 0 . Thus in a saddle node bifurcation, two fixed points having opposite stability properties coalesce to the origin, and disappear. Fig. C.2.b, which represents the qualitative features of this bifurcation for $\alpha$ and $x$ small, is called a bifurcation diagram. The curve on the left describes the two branches of fixed points. Arrows show where the discrete orbits generated by $G_{\alpha}$ go, for a given $\alpha$.

\section{Fig. C.2.a}

Fig. C.2.b

Proposition C.2.1. (Saddle node bifurcation). Let $G: U \times I \rightarrow R$ define a oneparameter family of maps, where $G$ is $C^{r}$ with $r \geqslant 2$, and $U, I$ are open intervals of the real line containing 0 . Assume

(1) $G(0,0)=0 ;(2) \frac{\partial G}{\partial x}(0,0)=1 ;(3) \frac{\partial^{2} G}{\partial x^{2}}(0,0)>0 ;(4) \frac{\partial G}{\partial \alpha}(0,0)>0$.

Then there are $\alpha_{1}<0<\alpha_{2}$ and $\varepsilon>0$ such that

(i) If $\alpha_{1}<\alpha<0$, then $G_{\alpha}=G(, \alpha)$ has two fixed points $x_{1 \alpha}<0<x_{2 \alpha}$ in $(-\varepsilon, \varepsilon)$. The fixed point $x_{1 \alpha}$ is asymptotically stable. the other is unstable. 
$\mid \begin{aligned} & n \\ & 0 \\ & i n \\ & i\end{aligned}$

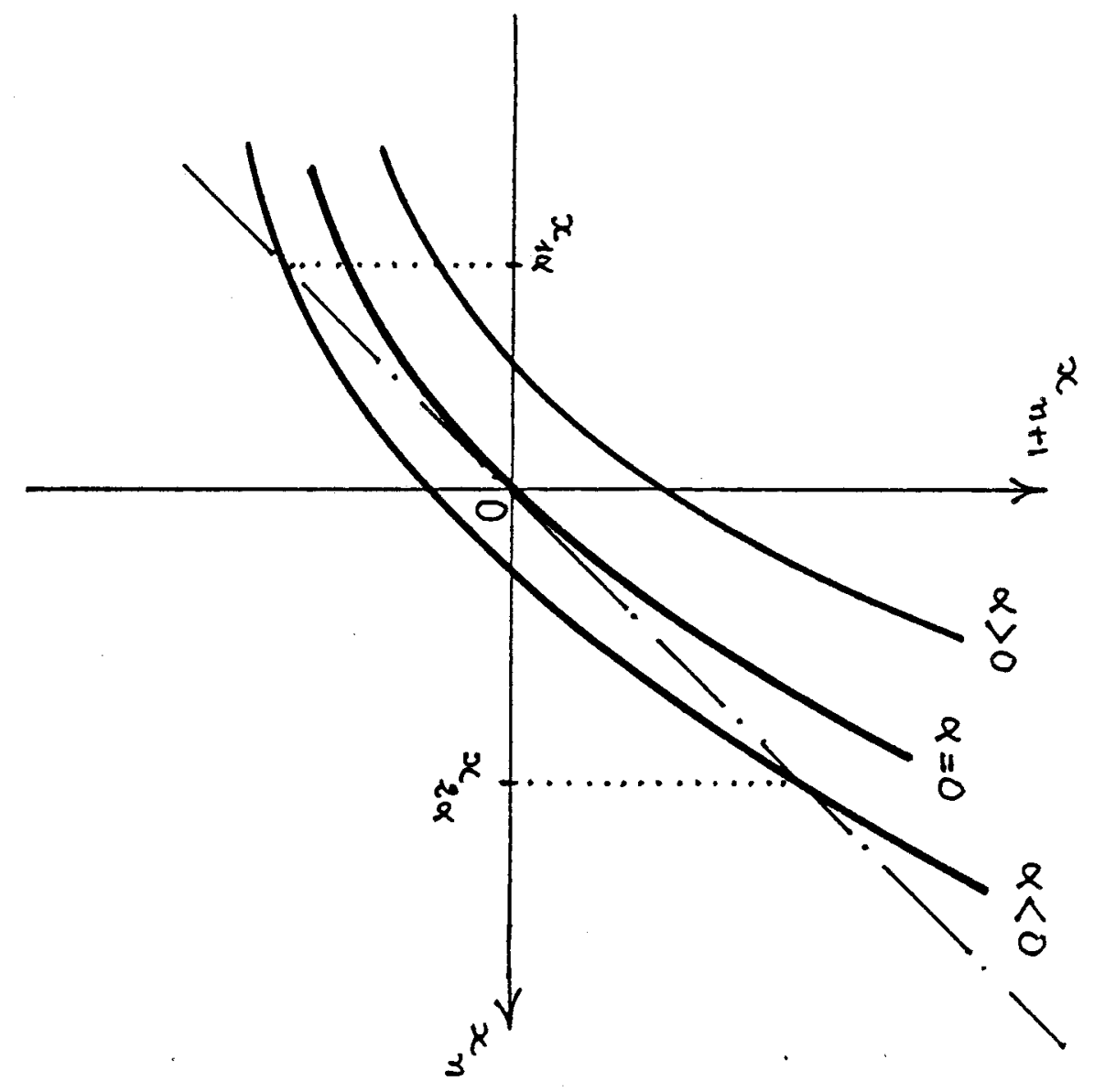

$\mid \pi$
$\mid n$
0
0

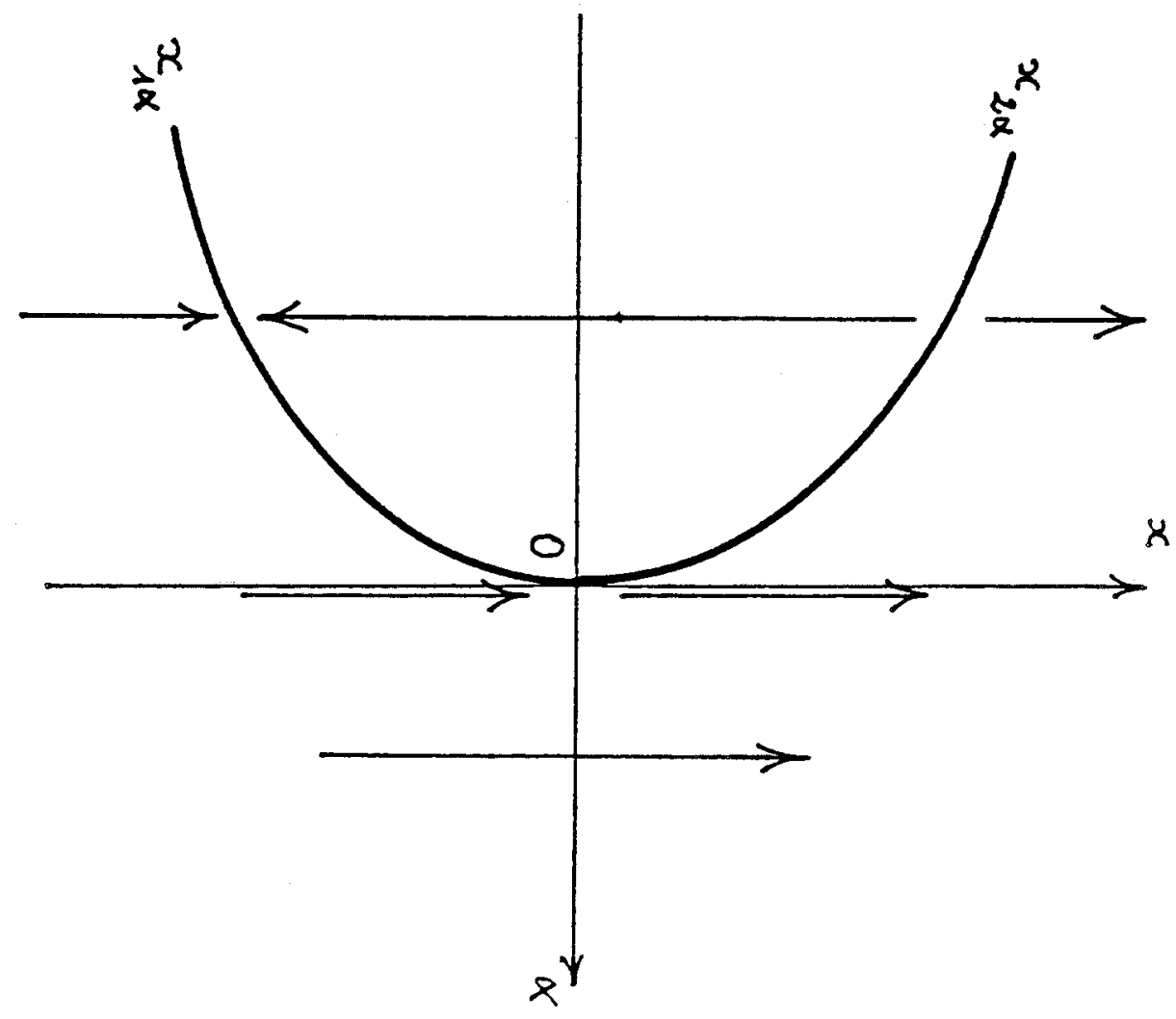


(ii) If $0<\alpha<\alpha_{2}$, then $G$ has no fixed point in $(-\varepsilon, \varepsilon)$.

It can be shown further that the curve on Fig. C.2.b describing the two branches of fixed points, i.e. the union of the sets $\left(x_{1 \alpha}, x_{2 \alpha}\right)$ for $\alpha,<\alpha \leq 0$, is indeed a $C^{r-1}$ onedimensional manifold that is tangent to the vertical axis at $x=0, \alpha=0$.

of course, the case $\frac{\partial G}{\partial \alpha}(0,0)<0$ is identical to the above after the change of parameter $\alpha \rightarrow-\alpha$. The reader will easily figure out what is the bifurcation diagram when inequality (3) is reversed: it amounts to changing the roles of positive and negative values of $\alpha$, and reversing the arrows in Fig. C.2.b.

\section{Iranscritical bifurcation}

The foregoing bifurcation described the "generic" situation. In some cases, however, the class of maps under consideration may be restricted, and a different bifurcation may occur. A common restriction is that the fixed point should persist, or equivalent7y, after a suitable $\alpha$-dependent change of variables that translates the fixed point to the origin, that 0 should be a fixed point for a 11 values of the parameter. In that case, one obtains a transcritical bifurcation.

Consider a family of maps defined by $G(x, \alpha)$ as above, with $\frac{\partial G}{\partial x}(0,0)=+1$. We require here that $G(0, \alpha)=0$ for all $\alpha$, and not only for $\alpha=0$ (this implies $\frac{\partial G}{\partial \alpha}(0,0)=0$, a violation of condition (4) of Proposition C.2.1), but keep the "generic" assumption $\frac{\partial^{2} G}{\partial x^{2}}(0,0) \neq 0$. In order to get 
actually a bifurcation, we must specify that $\frac{\partial G}{\partial x}(0, \alpha)$ goes through +1 when $\alpha$ passes 0 , that is $\frac{\partial^{2} G}{\partial \times \partial \alpha}(0,0) \neq 0$.

Fig. C.3.a describes what happens to the graphs of $G$ near 0 for $\alpha$ sma 17, when $\frac{\partial^{2} G}{\partial x^{2}}(0,0)>0$ and $\frac{\partial^{2} G}{\partial x \partial \alpha}(0,0)>0$. If $\alpha<0$, the origin is asymptotically stable, but there is another fixed point $x_{\mid \alpha}>0$ near 0 , which is unstable. If $\alpha>0$, the origin is unstable, and there exists another fixed point $x_{1 \alpha}<0$ near 0 , which is asymptotically stable. Thus in a transcritical bifurcation, there are two fixed points that exchange stability at the point of bifurcation. The corresponding bifurcation diagram is given in Fig. C.3.b.

\section{Fig. C.3.a}

Fig. C.3.b

Proposition C.2.2. (Transcritical bifurcation). Let $G: U \times I \rightarrow R$ define a oneparameter family of maps, where $G$ is $C^{r}$ with $r \geq 2$, and $U$, I are open intervals of the real line containing 0 . Assume

(1) $G(0, \alpha)=0$ for all $\alpha ; \frac{\partial G}{\partial x}(0,0)=1 ;(3) \frac{\partial^{2} G}{\partial x^{2}}(0,0)>0 ; \frac{\partial^{2} G}{\partial x \partial \alpha}(0,0)>0$.

Then there are $\alpha_{1}<0<\alpha_{2}$ and $\varepsilon>0$ such that

(i) If $\alpha_{1}<\alpha<0, G_{\alpha}$ has two fixed points, 0 and $x_{1 \alpha}>0$ in $(-\varepsilon, \varepsilon)$. The origin is asymptotically stable. the other fixed point is unstable.

(ii) If $0<\alpha<\alpha_{2}, G_{\alpha}$ has two fixed points, 0 and $x_{1 \alpha}<0$ in $(-\varepsilon, \varepsilon)$. The origin is unstable, the other fixed point is asymptotically stable.

It can be shown furthermore that each branch of fixed points in Fig. 
$\mid 1$
$\mid \begin{aligned} & n \\ & 0 \\ & 0 \\ & 0 \\ & 0\end{aligned}$
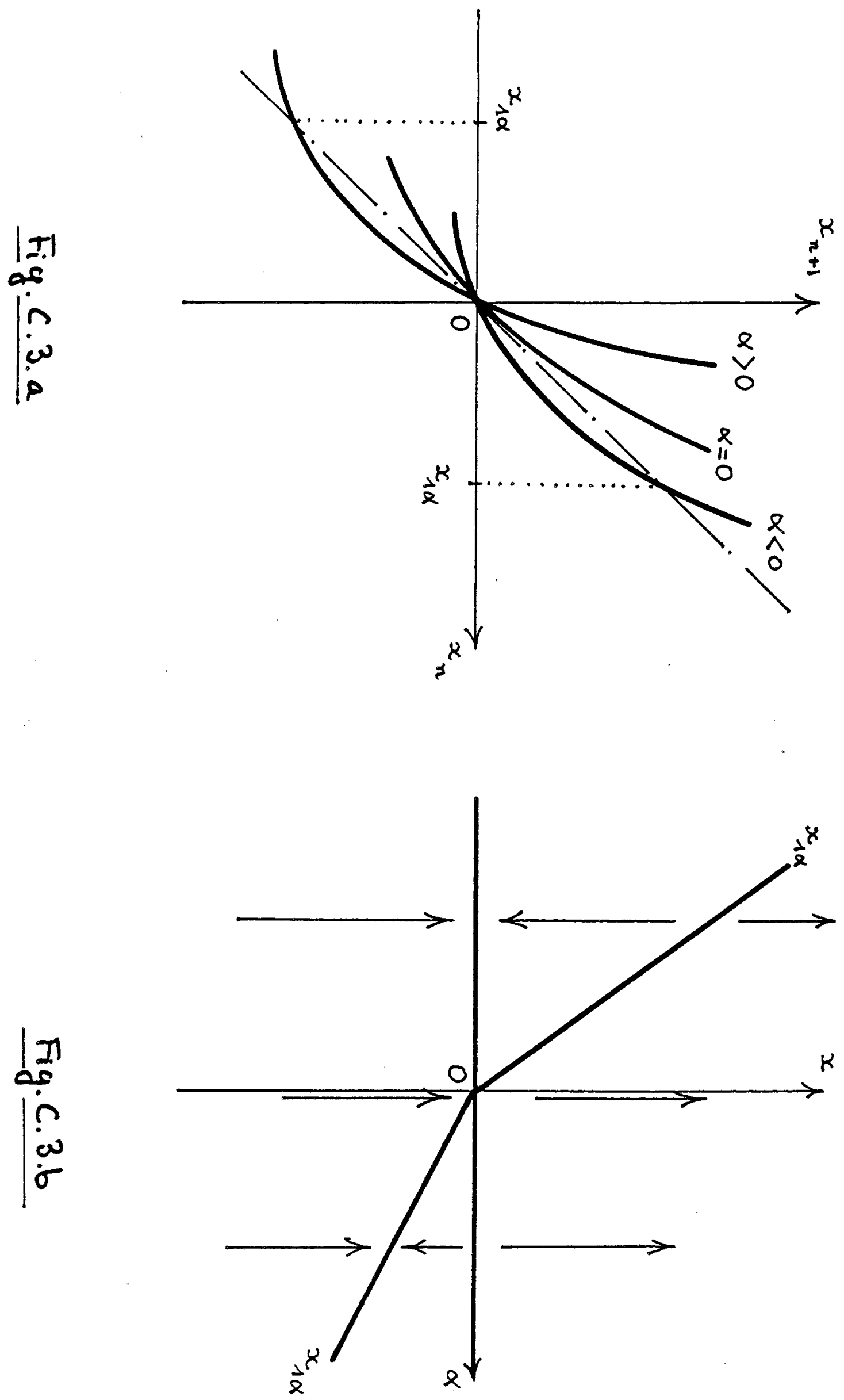
C.3.b, i.e. the union of all fixed points $x_{1 \alpha}$ for $\alpha_{1}<\alpha<0$, or for $0<\alpha<\alpha_{2}$, is a $C^{r-1}$ one dimensional manifold. Here again, the case $\frac{\partial^{2} G}{\partial x \partial \alpha}(0,0)<0$ is handled by making the change of parameter $\alpha \rightarrow-\alpha$. The reader will also figure out easily the bifurcation diagram when the inequality (3) is reversed : it amounts, here also, to changing the roles of positive and negative values of $\alpha$, and reversing the arrows in Fig. C.3.b.

\section{Pitchfork bifurcation}

Another interesting, "nongeneric" case arises when one assumes

$\frac{\partial^{2} G}{\partial x^{2}}(0,0)=0$ in the foregoing proposition. Let $G$ be $C^{3}$ and suppose that the third derivative, i.e. $\frac{\partial^{3} G}{\partial x^{3}}(0,0)$, differs from 0 . One gets then a pitchfork bifurcation.

Fig. C.4.a describes what happens to the graphs of $G_{\alpha}$ near the origin for $\alpha$ sma11, when $\frac{\partial^{3} G}{\partial x}(0,0)<0$. For $\alpha=0$, the graph of $G$ has an inflexion point at $x=0$, and the origin is asymptotically stable. If $\alpha<0, G$ has a unique fixed point, at $x=0$, near the origin, and it is asymptotically stable. If $\alpha>0, G$ has three fixed points near 0 . The origin is an unstable fixed point, but the two others are asymptotically stable. One says then that the family $G_{\alpha}$ undergoes a supercritical pitchfork bifurcation : the fixed point $x=0$ loses its stability as a goes through 0 , to give rise to a pair of stable fixed points. The corresponding bifurcation diagram is described in Fig. C.4.b. 
$\mid \begin{aligned} & n \\ & \dot{1} \\ & \dot{p} \\ & \dot{p} \\ & \dot{p}\end{aligned}$

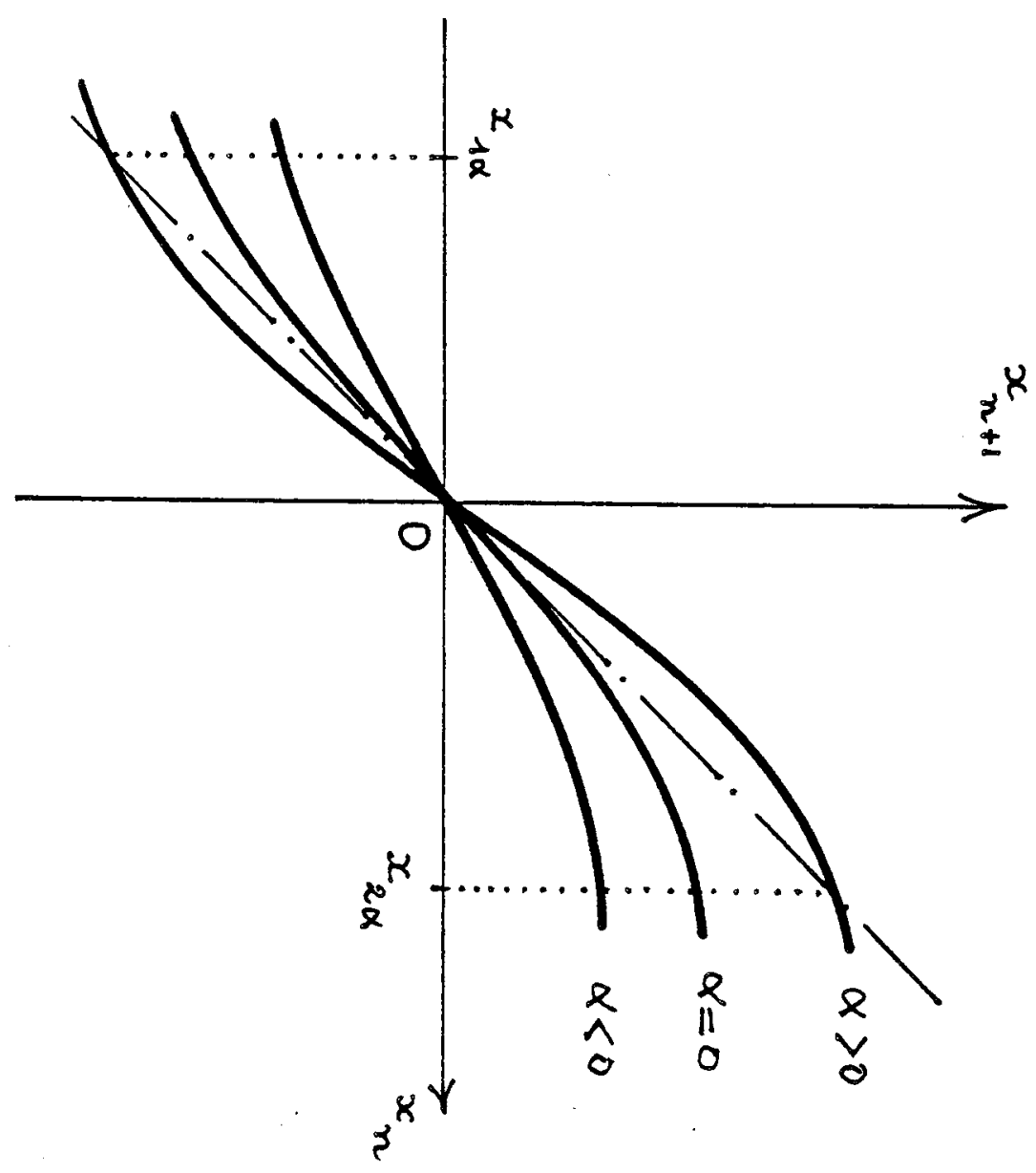

$n$
1
0
0
0

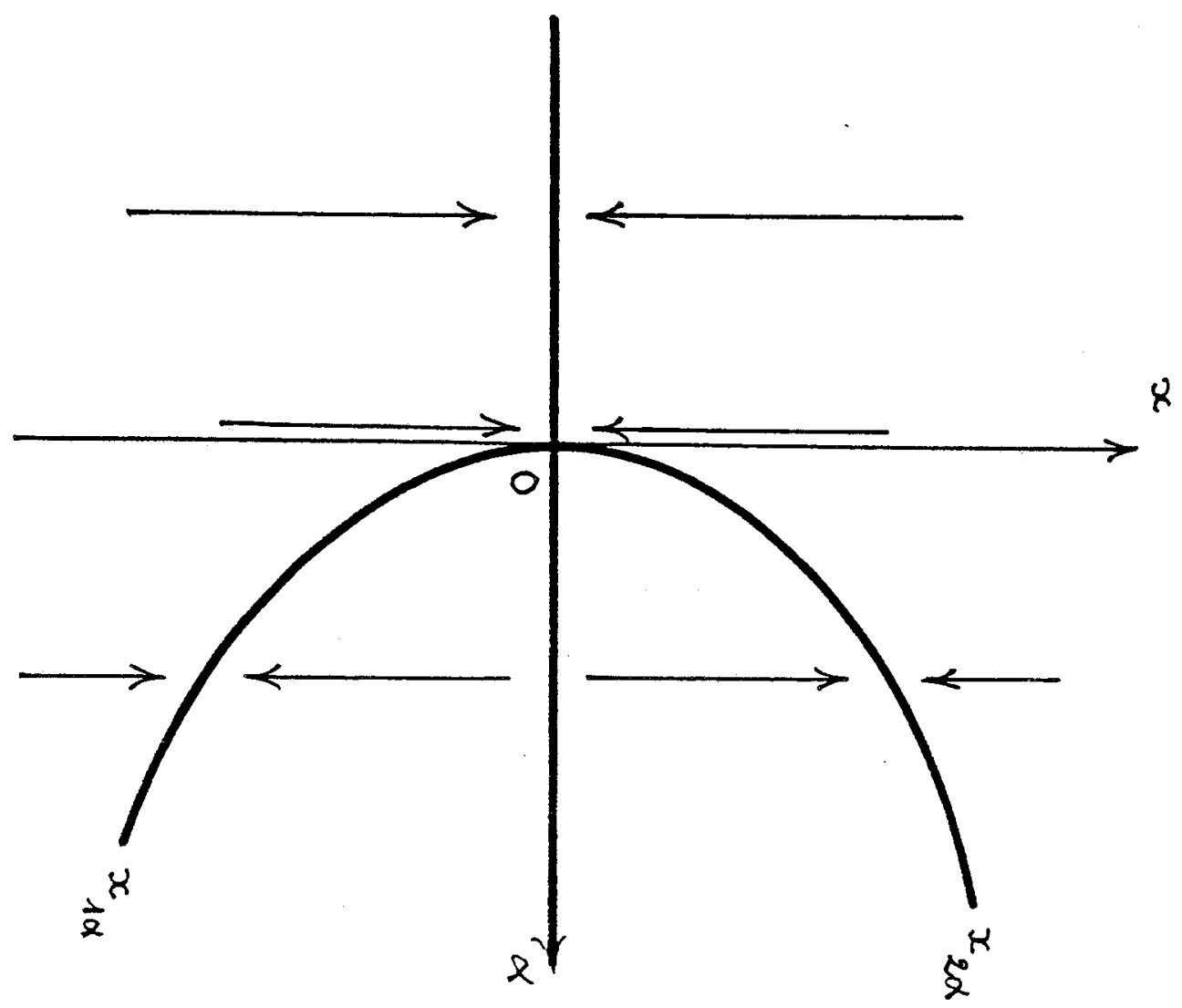


Proposition C.2.3. (Supercritical pitchfork bifurcation). Let $G: U \times I \rightarrow R$ be as in Proposition C.2.2, except that $G$ is $C^{r}$ with $r \geqslant 3, \frac{\partial^{2} G}{\partial x^{2}}(0,0)=0$ and $\frac{\partial^{3} G}{\partial x^{3}}(0,0)<0$. Then there are $\alpha_{1}<0<\alpha_{2}$ and $\varepsilon>0$ such that

(i) If $\alpha, \alpha \leq 0$, then $G$ has a unique fixed point, $x=0$, in $(-\varepsilon, \varepsilon)$. It is asymptotically stable.

(ii) If $0<\alpha<\alpha_{2}$, then $G$ has three fixed points in $(-\varepsilon, \varepsilon)$. The origin is an unstable fixed point. the two others, $x_{1 \alpha}<0<x_{2 \alpha}$, are asymptotically stable.

Here again, the two branches of fixed points in Fig.C.4.b, i.e. the union of the sets $\left(x_{1 \alpha}, x_{2 \alpha}\right)$ for $0 \leqq \alpha<\alpha_{2}$, is a $C^{r-1}$ onedimensional manifold that is tangent to $x$-axis at $x=0, \alpha=0$. The case $\frac{\partial^{2} G}{\partial x \partial \alpha}(0,0)<0$ is identical to the above after the change of parameter $\alpha \rightarrow-\alpha$. A more interesting modification arises when considering the case $\frac{\partial^{3} G}{\partial x^{3}}(0,0)>0$, which yields a socalled subcritical pitchfork bifurcation. What happens qualitatively is described in Fig. C.5.a, and the corresponding bifurcation diagram is given in Fig. C.5.b. At the bifurcation point $\alpha=0$, the graph of $G$ has an inflexion point at $x=0$ as before, but the origin is now unstable (whereas it was stable for $\alpha=0$ in the case of a supercritical bifurcation). Then for $\alpha<0$, there are three fixed points near the origin, but only $x=0$ is asymptotically stable. For $\alpha \geq 0$, the origin is the unique fixed point near $x=0$, and it is unstable. 
Remark C.2.4. The reader will easily verify that if the family 6 undergoes a subcritical pitchfork bifurcation when $\alpha$ goes through 0 from below, then the family of its local inverses $G_{\alpha}^{-1}$ undergoes a supercritical bifurcation when $\alpha$ goes through 0 from above. The family of local inverses is defined as follows. Since $\frac{\partial G}{\partial x}(0,0) \neq 0$, from the Implicit Function Theorem, the equation $G(x, \alpha)-y=0$ can be solved uniquely in $x$ for $x, \alpha, y$ small, to yield $x=F(y, \alpha)$ where $F$ may assumed to be defined on $V \times J$, with $V, J$ being two open intervals containing 0 . Then for every $\alpha$ in $J, F=F(\ldots, \alpha)$ is indeed a local inverse of $G_{\alpha}=G(\ldots, \alpha)$, and the family $F_{\alpha}$ undergoes a supercritical pitchfork bifurcation when a goes through 0 from above.

Remark C.2.5. It is clear that the qualitative features of the local bifurcations discussed here are preserved if one makes a change of parameter $\alpha \rightarrow \varphi(\alpha)$ and an $\alpha$-dependent change of variable $x \rightarrow h(x, \alpha)$, where $\varphi$ and $h(,, \alpha)$ are homeomorphisms. It can be shown that the converse result holds. For instance, two bifurcating families satisfying the assumptions of Proposition C.2.1 are locally topologically conjugate: they are the same, up to a change of parameter and a change of variable as above, at least sufficiently near $\alpha=0, x=0$. Similar statements hold for transcritical or pitchfork bifurcations. 

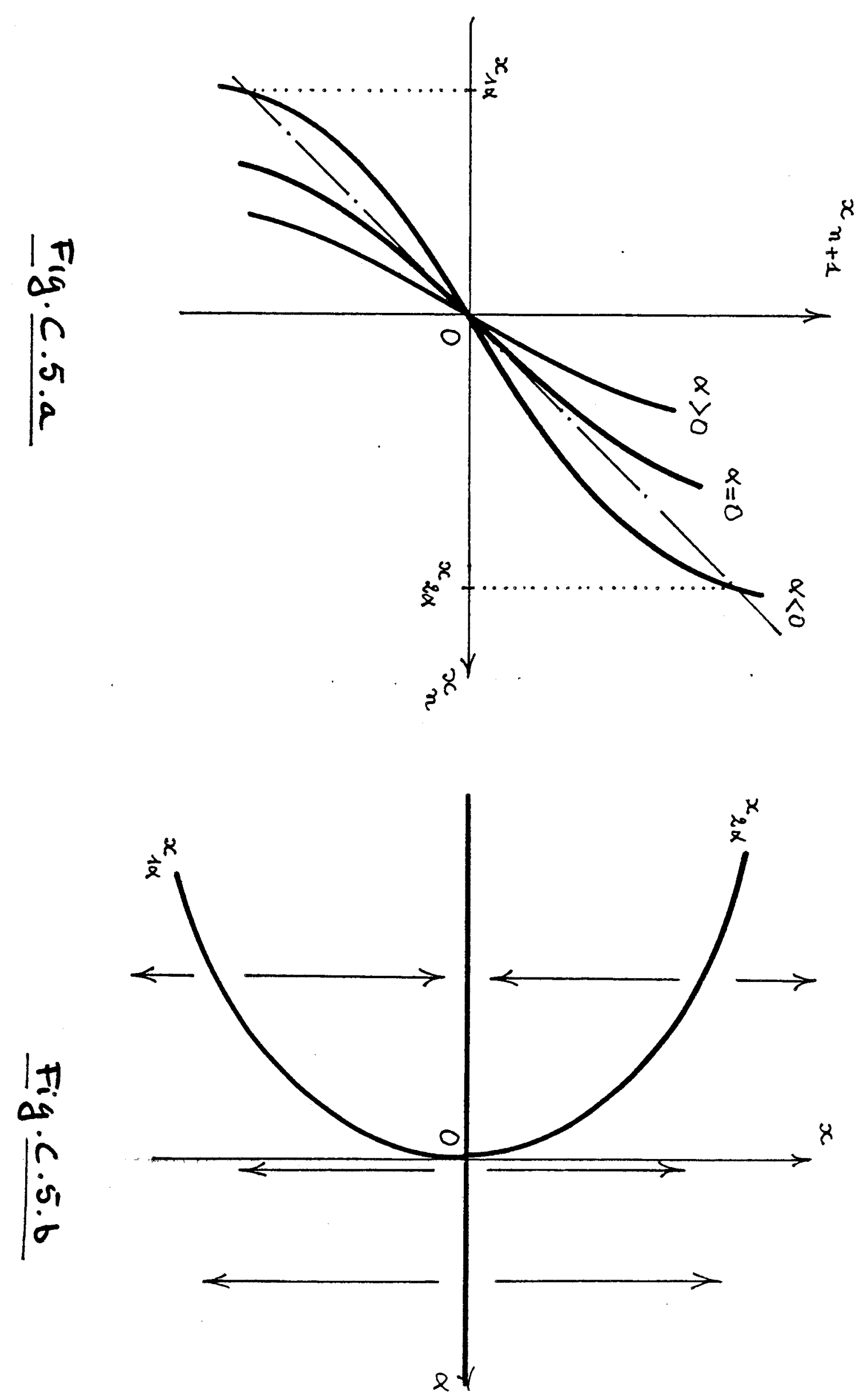
Finally, it should be noted that the bifurcations presented here are (locally) structurally stable, i.e. the qualitative features of the bifurcation are preserved, up to a change of variable, if the maps in the family are slightly perturbated (in a way that depends sufficiently smoothly on the parameter.). By contrast, as noted earlier, such structural stability could not obtain, had we assumed the maps in the family to be linear. 


\section{C.3. The flip bifurcation}

The previous section was devoted to the case where the one-parameter family of maps of the real line defined by $G(x, \alpha)$, was such that $\frac{\partial G}{\partial x}(0,0)=1$. We investigate now the case where $\frac{\partial G}{\partial x}(0,0)=-1$. To be specific, let the family be defined by the map $G: U \times I+R$ as before, where $G$ is $C^{3}$ (the need for continuous third derivatives will become apparent soon). Assume $G(0, \alpha)=0$ for all $\alpha, \frac{\partial G}{\partial x}(0,0)=-1$ and $\frac{\partial^{2} G}{\partial \alpha \partial x}(0,0)<0^{(2)}$. The origin $x=0$ is a fixed point of every $G_{\alpha}$, and the slope of the graph of $G_{\alpha}$, at the origin, decreases and passes through -1 as a goes through 0 from below. The family $G_{\alpha}$ undergoes then a flip bifurcation.

The orbits of $G_{\alpha}$ near the origin oscillate more and more around the fixed point $x=0$, when $\alpha$ varies from negative to positive values. If all the maps in the family $G$ were linear, then these oscillating trajectories would converge to 0 for $\alpha<0$, and diverge to infinity for $\alpha>0$, while every $x \neq 0$ would be a periodic point of period two for $\alpha=0$, since one would have then $G_{0}(x)=-x$ for al1 $x$. One should thus expect a cycle of period two to be part of the story in the more general case of nonlinear maps, as considered here.

The most efficient way to discover an orbit of period two of $G_{\alpha}$ is to look at its second iterate, $G_{\alpha}^{2}=G_{\alpha}$ oG $\alpha$, which is well defined near $x=0$. Indeed, a cycle of period two for $G_{\alpha}$ is characterized by a fixed point of $G_{\alpha}^{2}$ that differs from 0 .

Let us write $G_{\alpha}^{2}(x)$ as $G^{2}(x, \alpha)$. Since

$$
G^{2}(x, \alpha)=G(G(x, \alpha), \alpha)
$$


one has clearly $G^{2}(0, \alpha)=0$ for all $\alpha$, and one gets from the chain rule of differentiation

$$
\frac{\partial G^{2}}{\partial x}(0,0)=+1 ; \frac{\partial^{2} G^{2}}{\partial x \partial \alpha}(0,0)>0 ; \frac{\partial^{2} G^{2}}{\partial x^{2}}(0,0)=0 .
$$

If we suppose the "generic" condition $\frac{\partial^{3} G^{2}}{\partial x^{3}}(0,0) \neq 0$, we see that the family $G_{\alpha}^{2}$ undergoes a pitchfork bifurcation at $\alpha=0$ (3) . If $\frac{\partial^{3} G^{2}}{\partial x^{3}}(0,0)<0$, the bifurcation is supercritical, and what happens to the family $G_{\alpha}^{2}$ is qualitatively depicted in Fig. C.4. For $\alpha \leqq 0$, there is no cycle of period two near $x=0$, and the origin is a stable fixed point. When $a>0$, the origin becomes unstable, there is an orbit of period two near $x=0$, and this orbit is asymptotically stable (since a cycle of period two is characterized by a fixed point $\bar{x}$ of $G_{\alpha}^{2}$, asymptotic stability is defined by using $G_{\alpha}^{2}$, and is thus guaranteed when $\left.\left|\frac{\partial G^{2}}{\partial x}(\bar{x})\right|<1\right)$. Therefore, in a supercritical flip bifurcation, a stable fixed point becomes unstable, and gives rise to a stable orbit of period 2. For this reason, the flip bifurcation is sometimes called a period doubling (or subharmonic) bifurcation.

The corresponding bifurcation diagram for the family $G_{\alpha}$ itself is represented in Fig. C.6. The curve there describes the evolution of the two points on the orbit of period 2 when the parameter $\alpha$ varies. In Fig. C.7.a and $b$, the graphs of $G_{\alpha}$ are represented, as we 11 as the trajectories they generate near the origin, for $\alpha<0$ and $\alpha>0$ respectively. 


\section{Fig C.6}

Fig. C.7.a

Fig. C.7.b

The analogue of Proposition C.2.3 becomes here :

Proposition C.3.1 (Supercritical flip bifurcation). Let $G: U \times I \rightarrow R$ define a one-parameter family of maps, where $G$ is $C^{r}$, with $r \geq 3$, and $U$, I are open intervals containing 0 . Assume

(1) $G(0, \alpha)=0$ for all $\alpha ;$ (2) $\frac{\partial G}{\partial x}(0,0)=-1 ;$ (3) $\frac{\partial^{2} G}{\partial x \partial \alpha}(0,0)<0 ;(4) \frac{\partial^{3} G^{2}}{\partial x^{3}}(0,0)<0$.

Then. there are $\alpha_{1}<0<\alpha_{2}$ and $\varepsilon>0$ such that

(i) If $\alpha_{1}<\alpha \leq 0$, then $G$ has a unique fixed point at the origin and no orbit of period two in $(-\varepsilon, \varepsilon)$. The fixed point is asymptotically stable.

(ii) If $0<\alpha<\alpha_{2}$, then $G$ has a unique fixed point at the origin and a unique orbit of period two in $(-\varepsilon, \varepsilon)$. The fixed point is unstable and the orbit of period two is asymptotically stable.

In the same way as for the pitchfork bifurcation, the union of the period two points for $0 \leqq \alpha<\alpha_{2}$ in Fig. $C .6$, is a $C^{r-1}$ onedimensional manifold that is tangent to the $x$-axis at $x=0, \alpha=0$. When $\frac{\partial^{3} G^{2}}{\partial x^{3}}(0,0)>0$, the bifurcation is subcritical, and what happens to the family $G_{\alpha}^{2}$ is qualitatively described in Fig. C.5. The basic difference with the supercritical case is that the origin $x=0$ is now unstable when $\alpha=0$, while it was stable in the case of supercritical bifurcation. Thus in a subcritical flip bifurcation, an 

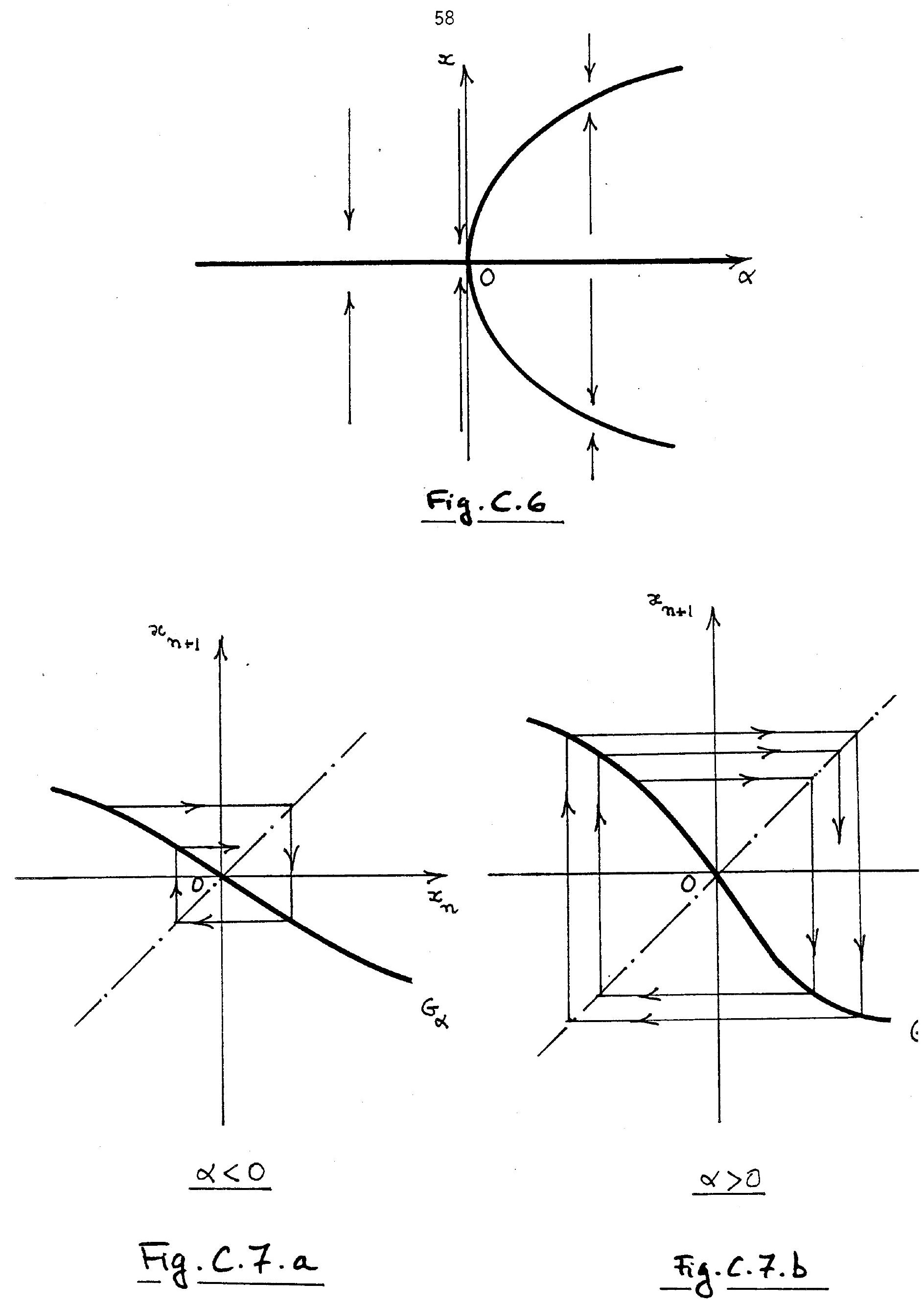
unstable orbit of period two coalesces with a stable fixed point at $\alpha=0$, to leave an unstable fixed point. The corresponding bifurcation diagram for the family $G_{\alpha}$ is given in Fig. C.8. Here again, the two branches of the curve represent the evolution of the two points of the orbit of period two. What happens to the graphs of the family $G_{\alpha}$ and to the trajectories they generate near the fixed point $x=0$ is described in Fig. C.9.a and b, for $\alpha<0$ and $\alpha>0$.

\section{Fig. C. 8}

$$
\text { Fig. C.9.2 Fig. C.9.6 }
$$

Proposition C.3.2 (Subcritical flip bifurcation). Replace the inequality (4) in Proposition $C .3 .1$ by $\frac{\partial^{3} G^{2}}{\partial x^{3}}(0,0)>0$. Then there exist $\alpha_{1}\left\langle 0<\alpha_{2}\right.$ and $\varepsilon>0$ such that

(i) If $\alpha_{1}<\alpha<0$, then $G$ has a unique fixed point at the origin and a unique orbit of period two in $(-\varepsilon, \varepsilon)$. The fixed point is asymptotically stable. the orbit of period two is unstable.

(ii) If $0 \leqq \alpha<\alpha_{2}$, then $G$ has a unique fixed point at the origin and no orbit of period two in $(-\varepsilon, \varepsilon)$. The fixed point is unstable.

Remark C.3.3. The Schwarzian derivative SG of a $C^{3}$ map $G: U \rightarrow R$ where $U$ is an open interval of the real line, is defined as

$$
S G(x)=\frac{G^{\prime \prime \prime}(x)}{G^{\prime}(x)}-\frac{3}{2}\left[\frac{G^{\prime \prime}(x)}{G^{\prime}(x)}\right]^{2}
$$

for every $x$ such that $G^{\prime}(x) \neq 0$. It is not difficult to verify that for the 


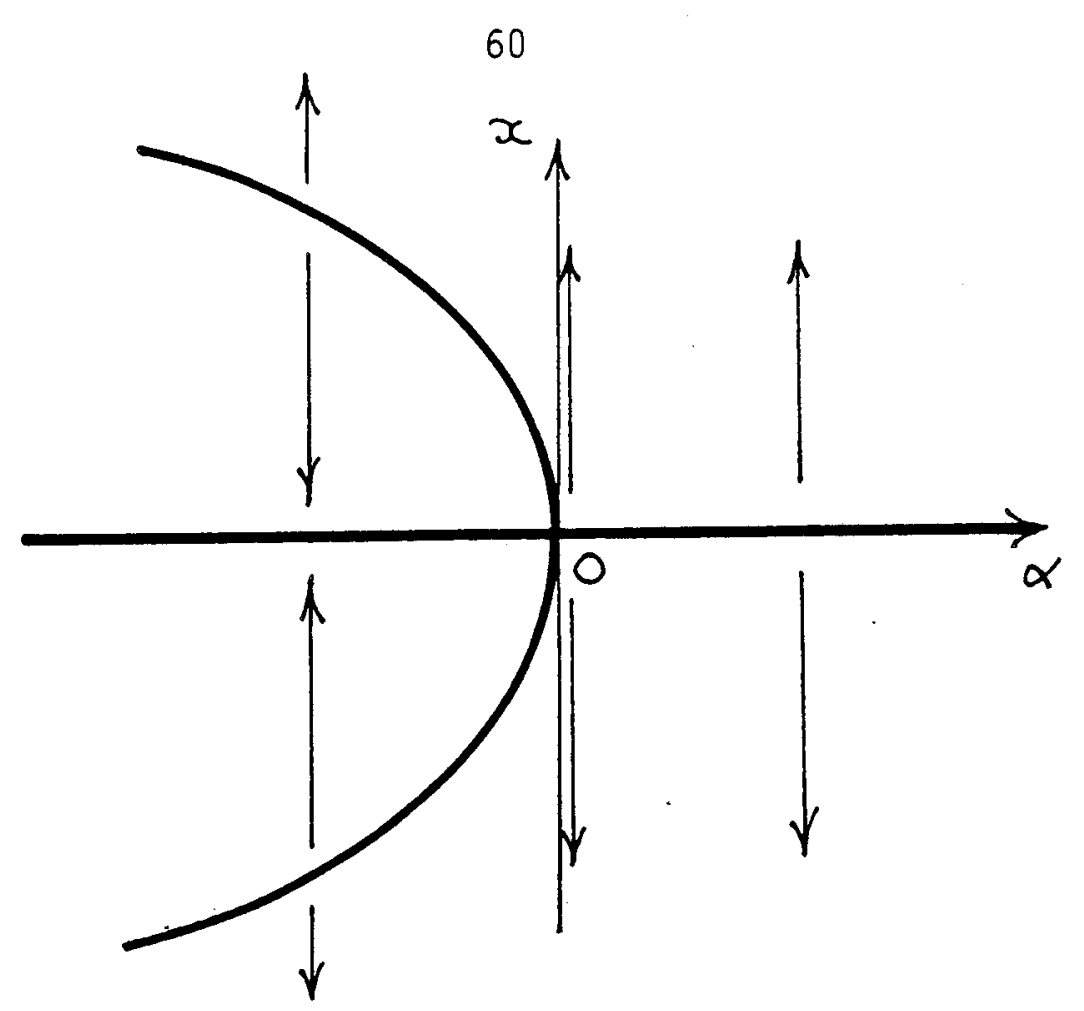

Fig.c. 8

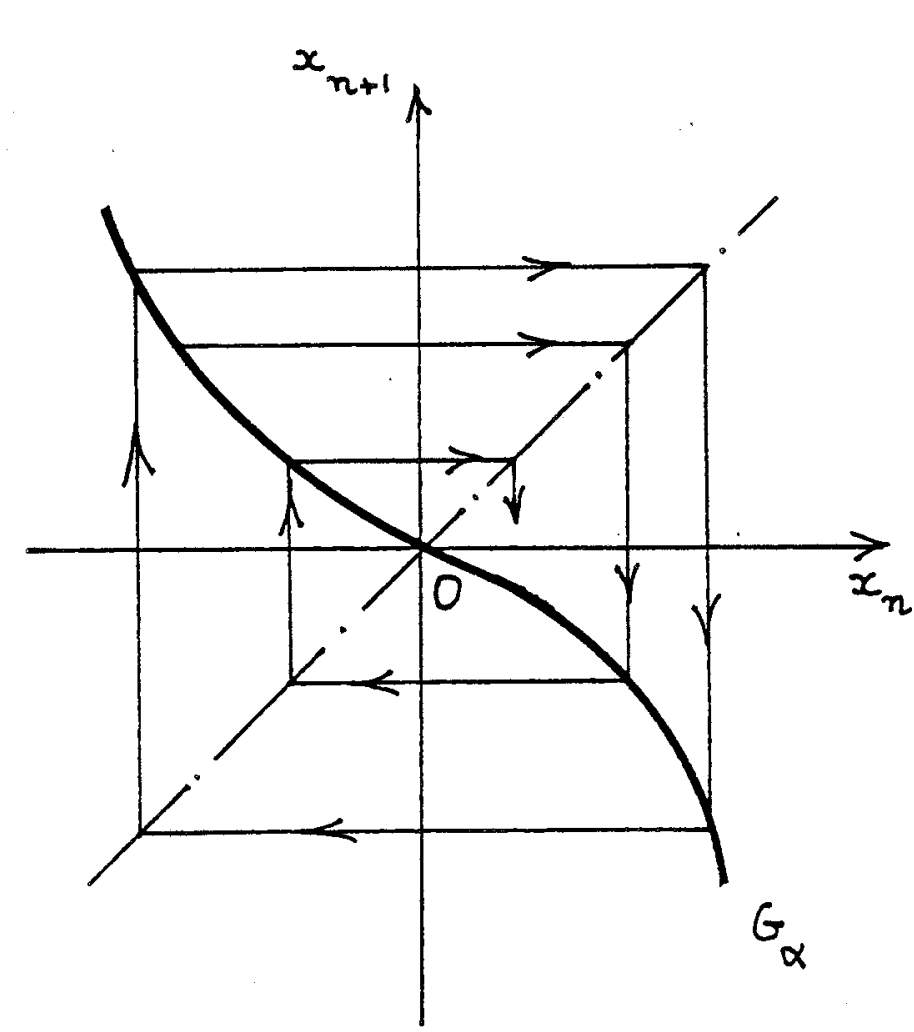

$\alpha<0$

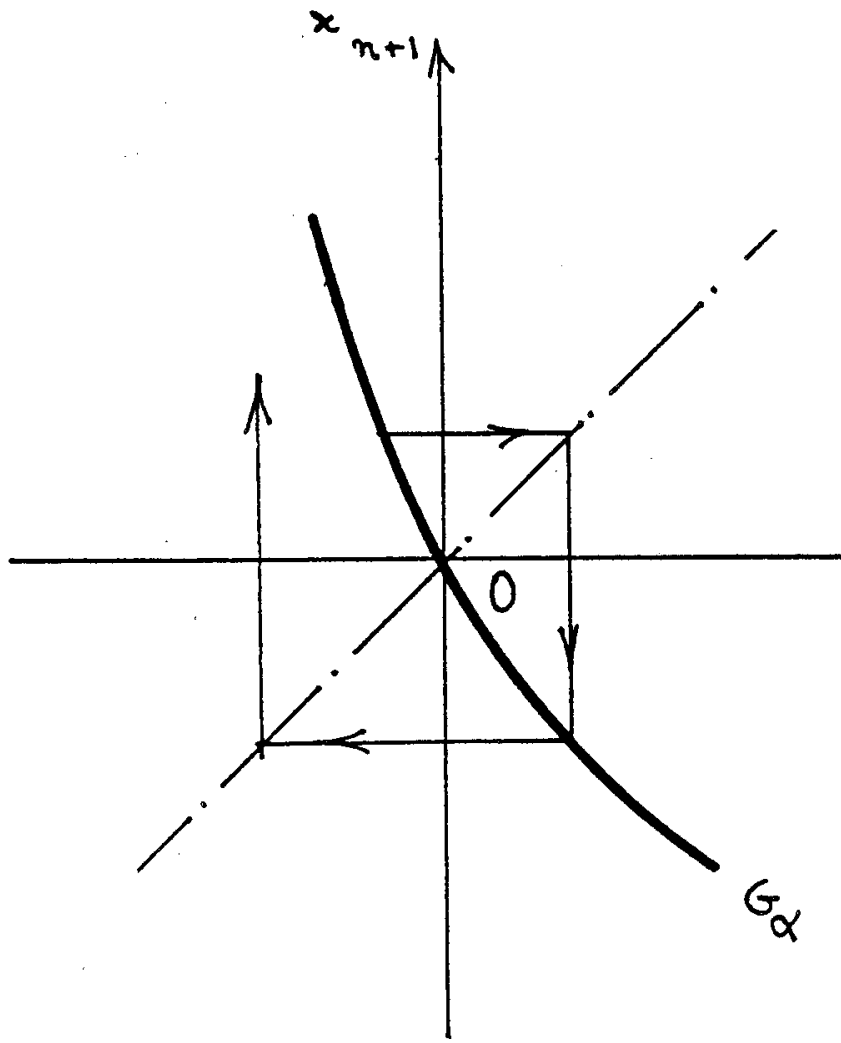

$\alpha>0$

Fig.C.9.a

Fig. C.9.6 
above family $G_{\alpha}$, one has

$$
\frac{\partial^{3} G^{2}}{\partial x^{3}}(0,0)=-2 \frac{\partial^{3} G}{\partial x^{3}}(0,0)-3 \frac{\partial^{2} G}{\partial x^{2}}(0,0)
$$

and since $\frac{\partial G}{\partial x}(0,0)=-1, \frac{\partial^{3} G^{2}}{\partial x^{3}}(0,0)=2 S G_{0}(0)$. It follows that condition (4) in Proposition C.3.1 may be expressed as : the Schwarzian derivative $5 G(x)$ is negative at $x=0$ at the point of bifurcation $\alpha=0$. Maps of the real line having globally a negative Schwarzian derivative have nice global properties, as we shall see in Appendix D, and their global bifurcations are reasonably well understood. The reason for this should be apparent from the preceding analysis : for such maps, a flip bifurcation is necessarily supercritical.

Remark C.3.4. As for the saddle mode bifurcation, two families undergoing a supercritical (or subcritical) flip bifurcation as in Proposition C.3.1 (or C.3.2) can be deduced from each other through a change of parameter $\alpha \rightarrow \varphi(\alpha)$, and an $\alpha$-dependent change of variable $x \rightarrow h(x, \alpha)$ where $\varphi$ and $h(, \alpha)$ are homeomorphisms. Similarly, the qualitative features of a flip bifurcation are (locally) structurally stable, up to such a change of variable. Here again, one could not get such a structural stability if the maps were linear.

\section{C.4. The Hopf bifurcation}

form

We consider now a one-parameter family of difference equations of the

$$
x_{n+1}=G\left(x_{n}, \alpha\right) \equiv G\left(x_{n}\right)
$$

defined by the $C^{r} \operatorname{map} G: U \times I \rightarrow R^{2}$, where $U$ is an open neighbourhood of the 
origin of $R^{2}$, and $I$ is an open interval of the real line containing 0 . We assume that the origin is a fixed point for all admissible values of the parameter, i.e. $G(0, \alpha)=0$ for al1 $\alpha$. We suppose further that for each $\alpha$, the Jacobian matrix $D_{x} G(0, \alpha)$ has a pair of nonreal, conjugate eigenvalues $\lambda(\alpha)$ and $\bar{\lambda}(\alpha)$, with $\lambda(\alpha)=\rho(\alpha)[\cos \theta(\alpha)+i \sin \theta(\alpha)], \rho(\alpha) \neq 0$ and $\theta(\alpha)$ in $(0, \pi)$. The modulus $\rho(\alpha)$ and the argument $\theta(\alpha)$ depend then in a $c^{r-1}$ fashion on the parameter. In order to get a bifurcation, we assume that $r>2$ and that the eigenvalues pass out of the unit circle in the complex plane as a goes through 0 , i.e. $e(0)=1$ and $\frac{d e}{d \alpha}(0)>0$. Then the origin $x=0$ is asymptotically stable for $\alpha<0$, unstable for $\alpha>0$ : there is a so-called Hopf bifurcation at $\alpha=0^{(4)}$.

From Section A.1, there is for each $\alpha$ a change of variable $x \rightarrow h(x, \alpha)$ which brings the Jacobian matrix $D_{x} G(0, \alpha)$ in its real canonical form

$$
{ }_{\alpha}=\rho(\alpha) \quad\left[\begin{array}{ll}
\cos \theta(\alpha) & -\sin \theta(\alpha) \\
\sin \theta(\alpha) & \cos \theta(\alpha)
\end{array}\right]
$$

the map $h$ being linear in $x$ and $c^{r-1}$ in $(x, \alpha)$. As noted in Section B.3, the action on $R^{2}$ of the linear map $y+B_{\alpha} y$ is a rotation of angle $\theta(\alpha)$, followed by an homothecy of center $y=0$ and ratio $\varrho(\alpha)$, see Fig. B.1. Thus the family of linear maps $B_{\alpha}$ can be represented in polar coordinates by $(\rho, \theta) \rightarrow(\rho(\alpha) \rho, \theta+\theta(\alpha))$. If $\alpha<0$, all trajectories generated by $y_{n}=B_{\alpha}^{n} y_{0}$ converge to the origin, while all such trajectories diverge when $y_{0} \neq 0$ for $\alpha>0$. By contrast, for $\alpha=0$, the map $y+B_{0}$ y leaves invariant 
every circle of the plane centered at the origin (of course, every such circle is generally an ellipse in the original coordinates).

Now if the maps in the family $G_{\alpha}$ are actually nonlinear, they are in fact small perturbations of the linear maps $B_{\alpha}$ near the origin. Thus one should expect the appearance of an invariant closed curve, i.e. a one-dimensional manifold that is homeomorphic to a circle of the plane, near $x=0$ and for $\alpha$ small. The following set of results shows that this is indeed in general the case.

We first look at the case where the bifurcation is supercritical. This case arises when the origin is attracting for $\alpha=0$. Then an attracting invariant closed curve appears for $\alpha>0$.

Theorem C.4.1 (Supercritical Hopf bifurcation). Let $G: U \times I \rightarrow R^{2}$ define a one-parameter family of maps of the plane, where $G$ is $C^{r}$ with $r \geq 6$, $U$ is an open set in $R^{2}$ containing the origin, and $I$ an open interval of the real line containing 0 . Assume

(1) $G(0, \alpha)=0$ for all $\alpha$;

(2) for each $\alpha, D_{x} G(0, \alpha)$ has a pair of nonreal, conjugate eigenvalues $\lambda(\alpha)$ and $\bar{\lambda}(\alpha)$, with $\lambda(\alpha)=\varrho(\alpha)[\cos \theta(\alpha)+i \sin \theta(\alpha)], \varrho(\alpha) \neq 0$ and $\theta(\alpha)$ in $(0, \pi) ;$

(3) $\rho(0)=1$ and $\frac{d e}{d \alpha}(0)>0$;

(4) $B(0) \neq 2 \pi / a$ for $q=1,2,3$ or 4 .

Then there is a $c^{r-3} \alpha$-dependent change of coordinates that brings the family $G_{\alpha}=G(,, \alpha)$ into the polar coordinates form 


$$
(\rho, \theta)+\left(\rho(\alpha) e-a(\alpha) e^{3}+e^{4} R(e, \theta, \alpha), \theta+\theta(\alpha)+b(\alpha) e^{2}+e^{3} S(e, \theta, \alpha)\right)
$$

where $R(0, \theta, \alpha)$ is $c^{r-4}, S(0, \theta, \alpha)$ is $c^{r-3}$, while $a(\alpha)$ and $b(\alpha)$ are $c^{r-3}$ and $c^{r-2}$, respectively. If in addition,

(5) $\mathrm{a}(0)>0$

then the origin is asymptotically stable for $\alpha=0$. Furthermore there exist $\alpha_{1}<0<\alpha_{2}$ and an open neighbourhood $V$ of the origin in $R^{2}$ such that

(i) If $\alpha_{1}<\alpha \leqq 0$, then $G$ has a unique fixed point at $x=0$, and no invariant closed curve in $V$. The origin is asymptotically stable.

( $i$ i) If $0<\alpha<\alpha_{2}$, then $G$ has a unique fixed point at $x=0$, and $a$ unigue closed invariant curve in $V$. The origin is unstable, while the invariant closed curve is asymptotically stable.

The first part of the theorem states that, if one puts aside the "strong resonance" cases $\theta=2 \pi / q, q=1,2,3$ or 4 (these cases are exceptional anyway), each map $G_{\alpha}$ may be approximated for $\times$ sma 11, after a suitable change of variables, by the transformation $T_{\alpha}$ given in polar coordinates by

$$
(0, \theta)+\left(e(\alpha) e-a(\alpha) e^{3}, \theta+\theta(\alpha)+b(\alpha) e^{2}\right)
$$

If the maps $G_{\alpha}$ are indeed nonlinear, one should have in general $a(0) \neq 0$ and $b(0) \neq 0$. The transformation $T_{\alpha}$ is the composition of a (a-dependent) rotation and of an homothecy. The contracting or expanding properties of the transformation are thus most easily analysed by looking directly at the associated onedimensional difference equation 


$$
e_{n+1}=e(\alpha) e_{n}-a(\alpha) e_{n}^{3} \equiv \gamma\left(e_{n}, \alpha\right)
$$

for $e_{n}, e_{n+1}>0$. In particular, $e_{\alpha}^{*}$ is a fixed point of $\gamma_{\alpha}=\gamma(\ldots, \alpha)$ if and only if $T_{\alpha}$ leaves invariant the circle of the plane centered at $x=0$ of radius $e_{\alpha}^{\star}$. Moreover, $e_{\alpha}^{*}$ is asymptotically stable in the dynamics generated by $\gamma_{\alpha}$ if and only if the corresponding invariant circle is asymptotically stable in the dynamics generated by $T_{\alpha}$.

It is easily seen that, if $a(0) \neq 0$ and if one allows negative values of e, the family $\gamma_{\alpha}$ undergoes a pitchfork bifurcation at $\alpha=0$. Indeed $\gamma$ is $c^{3}$, and for al1 $\alpha, \gamma(0, \alpha)=0, \frac{\partial \gamma}{\partial \rho}(0, \alpha)=e(\alpha), \frac{\partial^{2} \gamma}{\partial e^{2}}(0, \alpha)=0, \frac{\partial^{3} \gamma}{\partial e^{3}}(0, \alpha)=-6 a(\alpha)$. Thus if $a(0)>0$, the origin $e=0$ is asymptotically stable for $\alpha=0$, and the bifurcation is supercritical (Proposition C.2.3). What happens in that case to the graphs of $\gamma_{\alpha}$ near $e=0$, for a small, is represented in Fig. C.10.a. In terms of the dynamics associated with the transformation $T_{\alpha}$, for small $\alpha \leq 0$, the origin of $R^{2}$ attracts all nearby points. For small $\alpha>0$, the origin becomes unstable and $a 11$ small $x \neq 0$ in $R^{2}$ are attracted to an invariant circle centered at $x=0$, of radius $e_{\alpha}^{*}=\sqrt{(\rho(\alpha)-1) / a(\alpha)}$. The last part of the theorem states that this picture is qualitatively unchanged when one goes back to the original family $G_{\alpha}$. The corresponding bifurcation diagram is given in Fig. C. 11 . 

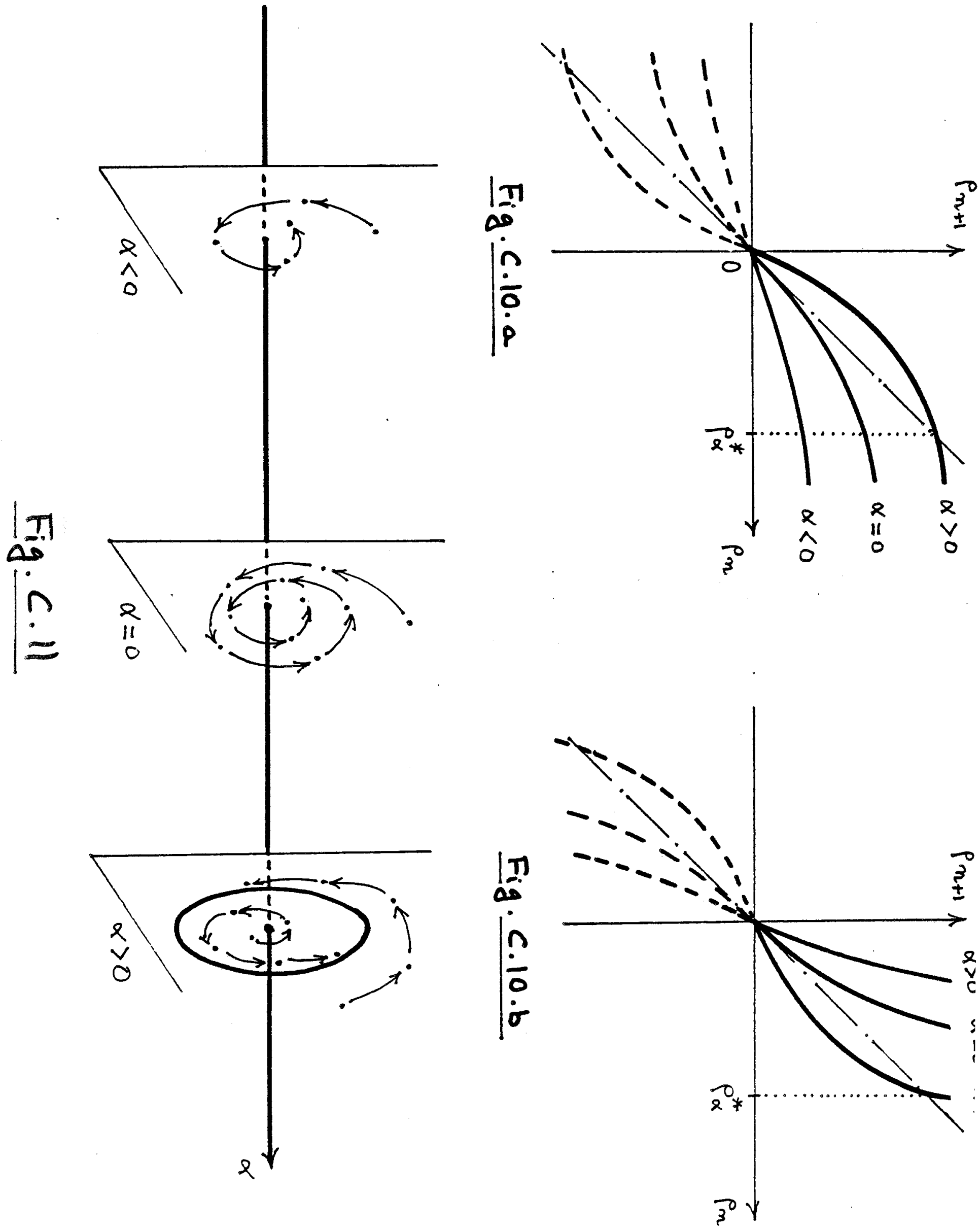
The same line of reasoning permits to see easily what happens when $a(0)<0$. In that case the origin is unstable at $\alpha=0$, and the family $\gamma_{\alpha}$ undergoes a subcritical pitchfork bifurcation (Fig. C.10.b). In terms of the dynamics associated with the transformation $T_{\alpha}$, for small $\alpha<0$, the origin is asymptotically stable, but there is a repelling invariant circle centered at $x=0$, of radius $e_{\alpha}^{*}=\sqrt{(\rho(\alpha)-1) / a(\alpha)}$. When $\alpha$ crosses 0 from below, the repelling invariant circle coalesces to the origin and for small $\alpha>0$, the fixed point $x=0$ is unstable. The following result states that the picture remains qualitatively the same when going back to the original family $G_{\alpha}$. The corresponding bifurcation diagram is given in Fig. C.12.

Theorem C.4.2 (Subcritical Hopf bifurcation). Assume in the preceding theorem that (5) is replaced by

$\left(5^{\prime}\right) a(0)<0$.

Then the origin is unstable for $\alpha=0$. Furthermore, there exist $\alpha_{1}<0<\alpha_{2}$, and an open neighbourhood $V$ of the origin in $R^{2}$ such that

(i) If $\alpha_{1}<\alpha<0$, then $G$ has a unique fixed point, at $x=0$, and a unique invariant closed curve in $V$. The origin is asymptotically stable, the closed curve is unstable.

(ii) If $0 \leqq \alpha<\alpha_{2}$, then $G$ has a unique fixed point at $x=0$, and no invariant closed curve in $V$. The or igin is unstable.

Fig. C. 12 


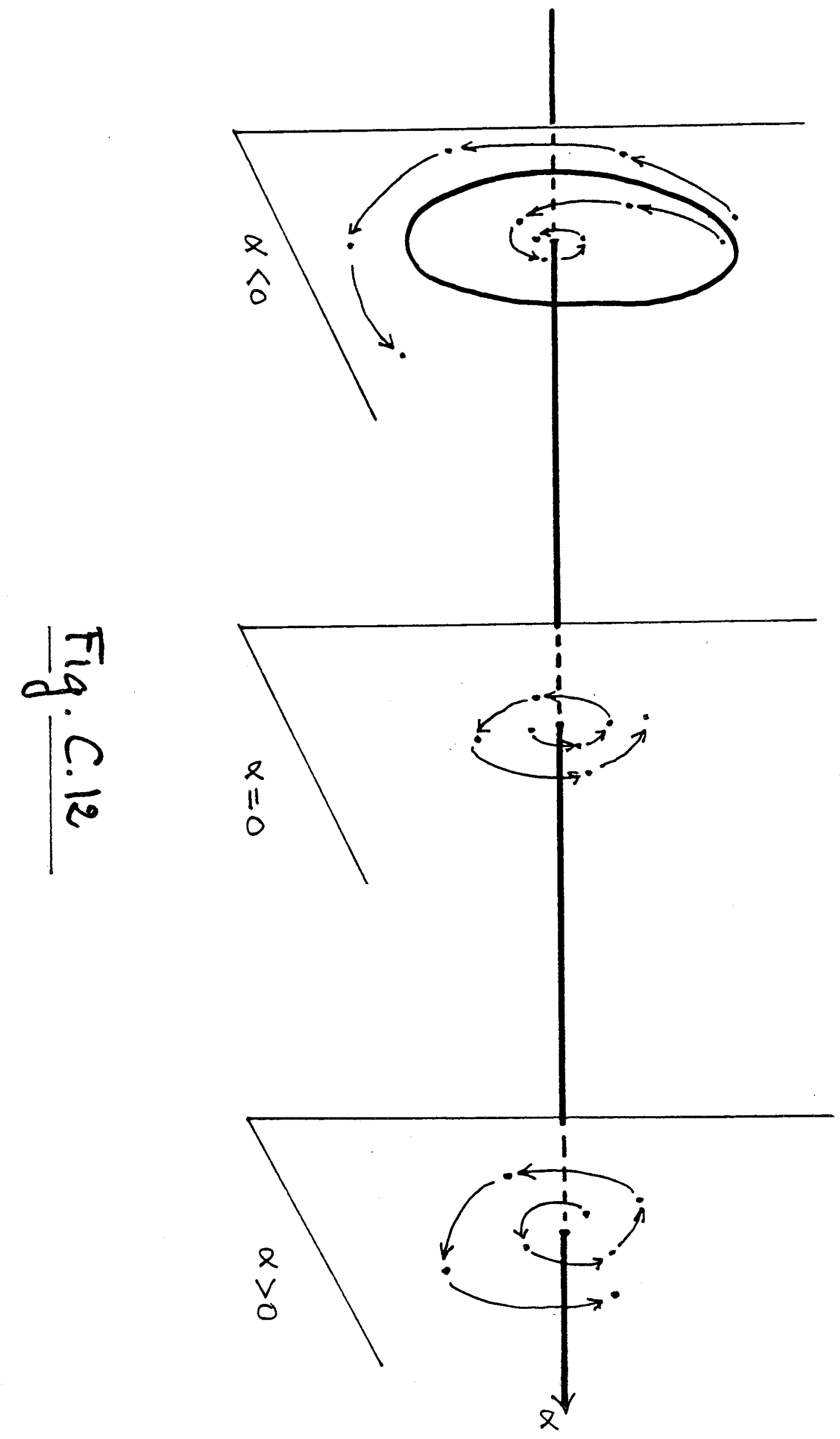


The sign of the coefficient a $(0)$ determines whether the bifurcation is supercritical or subcritical. The following proposition shows how to obtain in principle this coefficient - although the practical computations involved are sometimes tedious.

Proposition C.4.3. Under the assumptions of Theorem C.4.1, let $\lambda, \bar{\lambda}$ be the conjugate nonreal eigenvalues at $\alpha=0$, and assume that coordinates in the plane have been chosen to put $G_{0}$ in the form

$$
\left(\begin{array}{l}
x \\
y
\end{array}\right) \rightarrow\left[\begin{array}{rr}
\cos \theta(0) & -\sin \theta(0) \\
\sin \theta(0) & \cos \theta(0)
\end{array}\right]\left(\begin{array}{l}
x \\
y
\end{array}\right)+\left(\begin{array}{l}
f(x, y) \\
g(x, y)
\end{array}\right)
$$

Then one has

(5)

$$
a(0)=\operatorname{Re}\left[\frac{(1-2 \lambda) \lambda^{-2}}{1-\lambda} c_{11} c_{20}\right]+\frac{1}{2}\left|c_{11}\right|^{2}+\left|c_{02}\right|^{2}-\operatorname{Re}\left(\bar{\lambda} c_{21}\right),
$$

where

$$
\begin{aligned}
& 8 c_{20}=\left(f_{x x}-f_{y y}+2 g_{x y}\right)+i\left(g_{x x}-g_{y y}-2 f_{x y}\right) \\
& 4 c_{11}=\left(f_{x x}+f_{y y}\right)+i\left(g_{x x}+g_{y y}\right) \\
& 8 c_{02}=\left(f_{x x}-f_{y y}-2 g_{x y}\right)+i\left(g_{x x}-g_{y y}+2 f_{x y}\right) \\
& 16 c_{21}=\left(f_{x x x}+f_{x y y}+g_{x x y}+g_{y y y}\right)+i\left(g_{x x x}+g_{x y y}-f_{x x y}-f_{y y y}\right)
\end{aligned}
$$

in which the partial derivatives of $f$ and $g$ are evaluated at $(x, y)=(0,0)$.

Remark C.4.4. The invariant closed curve $c_{\alpha}$, of which the existence is asserted for $\alpha>0$ in Theorem C.4.1, may be represented as the locus of all points of the plane that have the polar coordinates $(\rho(\theta, \alpha), \theta)$, where $\theta$ is an 
arbitrary real number, $e(\theta, \alpha) \neq 0$ for all $\theta$ and $\alpha>0$, and $e$ is periodic of period $2 \pi$ in $\theta$, i.e. $\mathrm{e}(\theta+2 \pi, \alpha)=\mathrm{e}(\theta, \alpha)$. Under the assumptions of the Theorem, $\alpha_{2}$ can be chosen small enough so that the map a is $c^{r-5}$ for $0<\alpha<\alpha_{2}$. The union of all invariant closed curves $C_{\alpha}$ for $\alpha$ in $\left(0, \alpha_{2}\right)$, together with the origin of the plane for $\alpha=0$, form in the bifurcation diagram of Fig. C.11, a two dimensional $C^{r-5}$ manifold $[$ that is actualiy tangent to the $x$-plane at $\alpha=0$. This does not mean that $\sum$ is $C^{\infty}$ (is $C^{k}$ for al1 $k \geq 1$ ) when $G$ is itself $C^{\infty}$, however. In such a case, one can find $\alpha_{k}>0$ for each $k \geq 1$ such that $e$ and thus $\left[\right.$ is $C^{k}$ on $\left(0, \alpha_{k}\right)$. Yet the sequence $\alpha_{k}$ will typically tend to 0 as $k$ tends $+\infty$. The same regularity result holds for $\alpha<0$ in the case of a subcritical bifurcation.

Remark C.4:5. This remark is the analogue of Remark C.2.4 for. the case of a. pitchfork bifurcation. One can verify that if the family $G_{\alpha}$ undergoes a subcritical Hopf bifurcation when a goes through 0 from below, then the family of its local inverses $G_{\alpha}^{-1}$ undergoes a supercritical bifurcation when a goes through 0 from above. The family of local inverses is defined as follows. Since the eigenvalues of $D_{x} G(0,0)$ have modulus 1 , this matrix is invertible. From the Implicit Function Theorem, the equation $G(x, \alpha)-y=0$ can then be solved uniquely in $x$ for $x, \alpha, y$ small, to yield $x=F(y, \alpha)$ where $F$ may be assumed to be defined on $V \times J$, where $V$ is an open set of the plane containing the origin, and $\mathrm{J}$ is an open interval containing 0 . For every $\alpha$ in $J, F_{\alpha}=F(, \alpha)$ is indeed a local inverse of $G_{\alpha}=G(., \alpha)$, and the family $F_{\alpha}$ undergoes a supercritical bifurcation when a goes through 0 from above. 
Remark C.4.6. As noted earlier, the condition $a(0) \neq 0$ is generally satisfied (is "generic") in the class of all families of nonlinear maps, with enough differentiability. Analysis of the case $a(0)=0$ requires taking into account higher order terms in the approximating Taylor expansion $T_{\alpha}$ of the family $G_{\alpha}$, in polar coordinates. Analogues of Theorems $C .4 .1$ and $C .4 .2$ are actually available in the case $a(0)=0$, see Iooss (1979, Chapter III, Section 2).

The strong resonance cases $\theta(0)=2 \pi / q$ with $q=1,2,3,4$, are also "nongeneric" in the class of all nonlinear maps. The study of what happens in these cases involves some difficult and unsolved problems. For more information, see Whitley (1983, Section 2.5), and Iooss (1979, Chapter IV).

\section{Remark C.4.7 (Motion on the invariant closed curve)} obtained an asymptotically stable invariant closed curve $\mathrm{C}_{\alpha}$ in a supercritical Hopf bifurcation (for $\alpha>0$ in Theorem C.4.1), it remains to understand the behaviour of the trajectories generated by the difference equation $x_{n+1}=G_{\alpha}\left(x_{n}\right)$, when it is restricted to $c_{\alpha}$ (the results concerning a supercritical bifurcation can then be transposed to the subcritical case, for instance by using Remark $(.4 .5)$.

Under the assumptions of Theorem C.4.1, for small $\alpha$, the map $G_{\alpha}$ is only a small perturbation, after a suitable change of variables, of the transformation $T_{\alpha}$ given in polar coordinates by

$$
(g, \theta) \rightarrow\left(g(\alpha) \rho-a(\alpha) e^{3}, \theta+\theta(\alpha)+b(\alpha) e^{2}\right)
$$

Thus for sma11 $\alpha>0$, in this system of polar coordinates, the restriction of $G_{\alpha}$ to the invariant closed curve $\mathrm{C}_{\alpha}$ is a small perturbation of the restriction 
to the circle centered at the origin of radius $e_{\alpha}^{*}=\sqrt{(0(\alpha)-1) / a(\alpha)}$, of a rotation $R_{\alpha}$ of angle $\theta_{\alpha}^{*}=\theta(\alpha)+b(\alpha)\left(\rho_{\alpha}^{*}\right)^{2}$.

Let us first look at the dynamics generated by $R_{\alpha}$. If $\theta_{\alpha}^{\star} / 2 \pi$ is rational, i.e. of the form $p / q$ where $p$ and $q$ are positive integers that are relatively prime, every point of the circle is periodic with period a (i.e. one goes back to it when applying a-times the rotation $R_{\alpha}$ ). If $\theta_{\alpha}^{*} / 2 \pi$ is irrational, then no point of the circle is periodic : the trajectory generated by an arbitrary point of the circle is dense in that circle.

Knowledge of the properties of the restriction of the approximating rotation $R_{\alpha}$ to the invariant circle of radius $e_{\alpha}^{*}$ is not enough, unfortunately, to state the properties of the original family $G_{\alpha}$ restricted to the invariant closed curves $C_{\alpha}$. The reason is that such rotations $R_{\alpha}$ are not structurally stable : the fact that $\theta_{\alpha}^{*} / 2 \pi$ is rational or irrational can be altered by a sma 11 perturbation of the map. It can be shown nevertheless that, as far as the behaviour of the restriction of $\mathrm{G}_{\alpha}$ to $\mathrm{C}_{\alpha}$ is concerned, the typical (i.e. "generic") picture is the following one. Assuming that $G$ is $C^{\infty}$, and if the parameter $\alpha_{2}$ appearing in Theorem $C .4 .1$ is chosen small enough

1. there is a set $A$ that is open and dense in $\left(0, \alpha_{2}\right)$ such that for every $\alpha$ in $A$, the map $G$ has two families of periodic orbits, all having the same (a-dependent) period. on the invariant closed curve $\mathrm{C}_{\alpha}$. One family is stable, the other unstable.

The best way to visualise what happens is to look at the path followed in the complex plane by the eigenvalue $\lambda(\alpha)$ of the Jacobian matrix 
$D_{x} G(0, \alpha)$, as shown in Fig. C.13.a. Consider a complex number of the form $\cos \theta+i \sin \theta$ represented by the point $M$ on the unit circle, with $\theta=2 \pi p / q$, where $p$ and $q$ are positive integers that are relatively prime, and $q \neq 1,2,3,4$ (again, we put aside the strong resonance cases). Then there is a narrow cusped open region, near the unit circle, that lies between two curves passing through $M$ and having a common tangent there as in the hatched region of $F$ ig. C.13.a, with the following property. Suppose that the point representing the eigenvalue $\lambda(\alpha)$ lies in this narrow "tongue" for $\alpha>0$ in some interval $\left(\alpha^{\prime}, \alpha^{\prime \prime}\right)$. Then for every $\alpha$ in that interval, $G$ has two families of periodic orbits, of period $a$, on the invariant closed curve $\mathrm{C}_{\alpha}$. The periodic orbits of one family are unstable, while the periodic orbits of the other family are asymptotically stable (in the dynamics generated by the restriction of $G_{\alpha}$

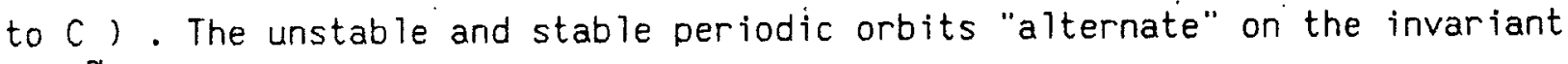
closed curve; an example of such a pattern is given in Fig. C.13.b, with $a=6$ (the arrows indicate stability or unstability; they are drawn as if the trajectories were continuous, the interpretation in discrete time should be apparent).

\section{Fig C.13.a Fig. C.13.b}

Since rational numbers are dense in the real line, the path followed by the eigenvalue $\lambda(\alpha)$ in the complex plane will cross infinitely many such distinct little tongues. The union A of the corresponding intervals $\left(\alpha^{\prime}, \alpha^{\prime \prime}\right)$ of the parameter will be open and dense in $\left(0, \alpha_{2}\right)$ if $\alpha_{2}$ is small enough. Thus for most values of $\alpha$ in $\left(0, \alpha_{2}\right)$, the asymptotic behaviour of the trajectories 
74
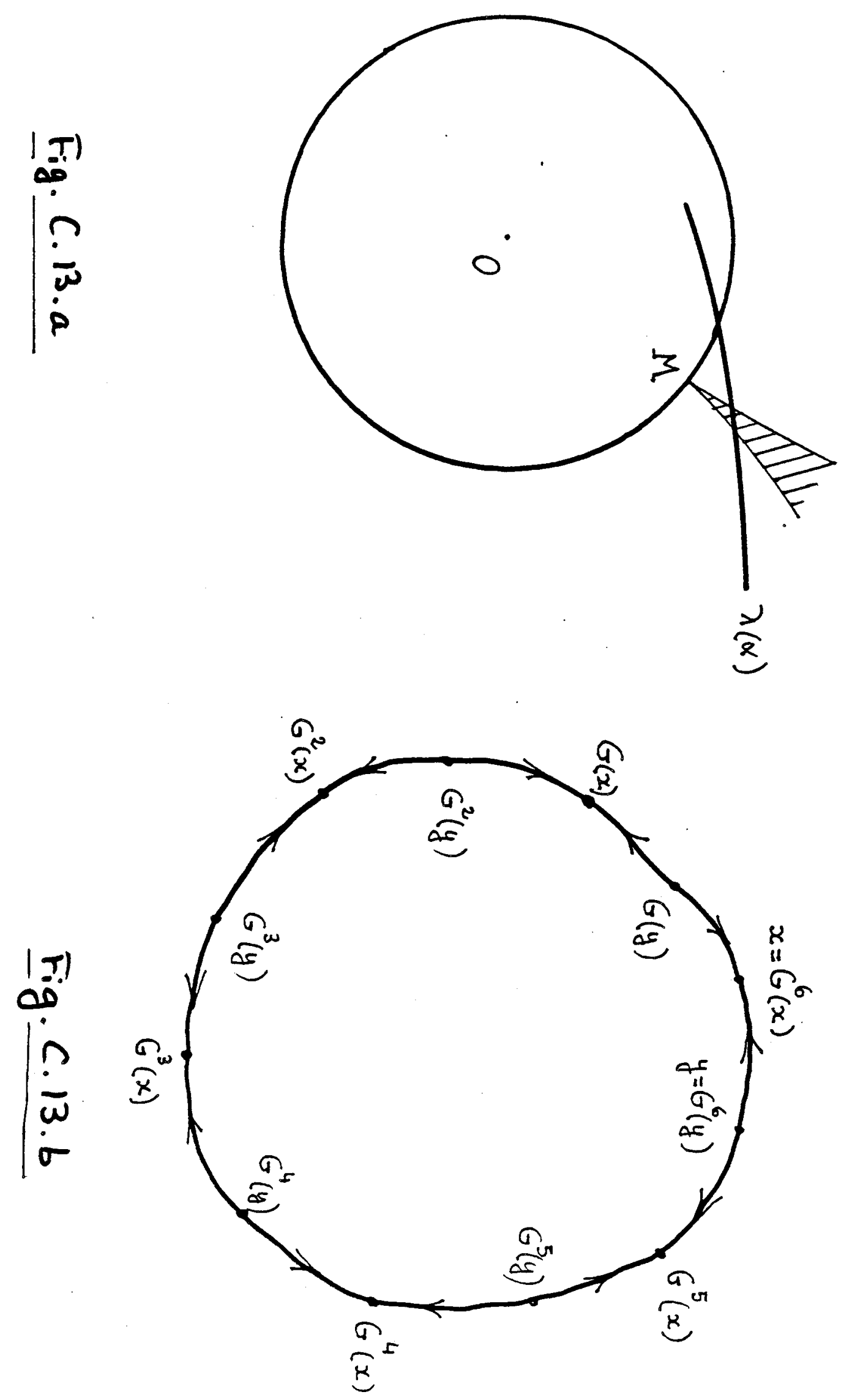
generated by $G$ on the invariant closed curve will be periodic. It should be remarked that many of the periods will be large, i.e. will correspond to a large $q$.

2. Although the above mentioned set $A$ is open and dense in $\left(0, \alpha_{2}\right)$, its complement in that interval is not negligible from a measure theoretic

viewpoint. Indeed, the set $B$ of parameters in $\left(0, \alpha_{2}\right)$ such that the restriction of $G_{\alpha}$ to the invariant closed curve $C_{\alpha}$ is topologically conjugate to a rotation of angle $\theta$ of the unit circle of the plane, with $\theta / 2 \pi$ irrational, has positive Lebesgue measure $m(B)>0$. In that case, any orbit generated by the restriction of $G_{\alpha}$ to $C_{\alpha}$ is aperiodic : it is dense in $C_{\alpha}$. In fact, the relative frequency of such aperiodic behaviour is high for $\alpha>0$ small: the ratio $m(B) / \alpha_{2}$ tends to 1 when $\alpha_{2}$ goes to 0 .

Remark C.4.8. It should be clear from the previous remark that the qualitative features of a Hopf bifurcation cannot be structurally stable. After a perturbation, the path followed by the eigenvalue $\lambda(\alpha)$ would cross the unit circle at a different point, and thus meet different little tongues as in Fig. C.13.a. The two families could not be conjugate.

\section{C.5. The center manifold reduction}

Local bifurcations appear to be hard to analyse in $R^{m}$, if $m$ is large. Fortunately, as mentioned in Section C.1, an application of the theory of center manifolds permits to reduce the dimension of the problem to the number of eigenvalues the modulus of which goes through 1 in the bifurcating family. We exploited that fact in the previous sections by looking at the saddle node or the flip bifurcations directly for maps of the real line, and by analysing 
the Hopf bifurcation directly in the plane. We show now precisely how to implement such a reduction of the dimension of the problem.

Consider a family of difference equations defined by the map $G: U \times I+R^{m}$, where $U$ is an open set of $R^{m}$ containing the origin, $I$ is an open interval of the real line containing 0 , and $G$ is $C^{r}$, with $r \geq 2$. We assume that the origin of $R^{m}$ is a fixed point of each map $G_{\alpha}=G(\ldots, \alpha)$ of the family, i.e. $G(0, \alpha)=0$ for all $\alpha^{(7)}$. We let $A_{\alpha}$ be the Jacobian matrix $D_{X} G(0, \alpha)$, and assume that $A_{0}$ is invertible and has some eigenvalues of modulus 1. The family will thus generally undergo a local bifurcation (near the origin) at $\alpha=0$. We assume furthermore that a linear change of variables has brought $A_{0}$ in its real canonical form, i.e. $A_{0}=\operatorname{diag}(C, B)$, in which $C$ correspond to the center space $F^{C}$ of $A_{0}$, and $B$ to the direct sum of the stabie and unstable spaces $F^{S}$ and $F^{U}$ of $A$. We shall write accordingly $x=(\varepsilon, n)$, where $E$ belongs to $F^{c}$ and $n$ to $F^{s}+F^{u}$.

It is convenient here to consider that the map $G$ defines a single difference equation in $R^{m+1}$ by

$$
\alpha_{n+1}=\alpha_{n}, x_{n+1}=G\left(x_{n}, \alpha_{n}\right)
$$

The trajectory generated by this difference equation, that corresponds to the initial condition $\left(\alpha, x_{0}\right)$, or more precisely its projection on the $x$-space, coincides then to the orbit of $x_{0}$ generated by the map $G_{\alpha}$. Analysing the local bifurcation of the family $G_{\alpha}$ near $x=0$, for $\alpha$ small, is thus equivalent to studying the local properties of the map $H: I \times U \rightarrow R^{m+1}$ defined by

$$
(\alpha, x) \longrightarrow(\alpha, G(x, \alpha))
$$


near its fixed point $\alpha=0, x=0$. One can then apply the local analysis developed in Section B.5.

Since $G(0, \alpha)=0$ for all $\alpha$, the partial derivatives of $G$ with respect to $\alpha$, evaluated at $x=0, \alpha=0$, are equal to zero. Thus the Jacobian matrix $\mathrm{DH}(0,0)$ is block diagonal and given by

$$
D H(0,0)=\left[\begin{array}{lll}
1 & & \\
& c & \\
& & 0
\end{array}\right]
$$

The stable and unstable spaces of $\mathrm{DH}(0,0)$ are thus equal to $(0) \times \mathrm{F}^{\mathrm{s}}$ and $\{0\rangle \times F^{u}$, respectively, while its center space is $R \times F^{C}$.

According to Theorem B.5.1, there is a neighbourhood $V$ of $(0,0)$ in $R^{m+1}$ such that $H$ has a local center manifold $W_{(0,0)}^{c}$ in $V$. Such a center manifold is $C^{r-1}$ and has the same dimension as the center space of $\mathrm{DH}(0,0)$, i.e. $R \times F^{C}$, to which it is tangent at $\alpha=0, \xi=0, \eta=0$. More importantly, it is locally attracting : if $(\alpha, x)$ and $H^{n}(\alpha, x)$ are in $V$ for a $11 n \geqslant 1$, then the distance between $H^{n}(\alpha, x)$ and $W_{(0,0)}^{c}$ tends to 0 as $n \rightarrow+\infty$. This fact implies that any recurrent behaviour that may appear. or disappear. in the bifurcating family, such as fixed points, periodic orbits, invariant closed curves, must Iie in a center manifold $W_{(0,0)}^{c}$. This result is the key to the reduction of the dimension of the problem.

To be specific, assume that the difference equation associated with $H$, has the following representation

$$
\begin{aligned}
& \alpha_{n+1}=\alpha_{n} \\
& \xi_{n+1}=C \xi_{n}+f\left(\xi_{n}, n_{n}, \alpha_{n}\right)
\end{aligned}
$$




$$
n_{n+1}=B \eta_{n}+g\left(\varepsilon_{n}, \eta_{n}, \alpha_{n}\right)
$$

in which $f$ and $g$ are defined for $(\varepsilon, \eta) \in U, \alpha \in I$. Of course the last two lines stand for $x_{n+1}=G\left(x_{n}, \alpha_{n}\right)$. So, by construction, the functions $f$ and $g$ are $c^{r}, r \geq 2, f(0,0, \alpha)=0$ and $g(0,0, \alpha)=0$ for all $\alpha$, and the first partial derivatives of $f$ and $g$ with respect to $\varepsilon$ and $\eta$, vanish at $\varepsilon=0$, $\eta=0, \alpha=0$.

In this system of coordinates, a local center manifold $W_{(0,0)}^{c}$ can be represented as the graph of a $C^{r-1}$ function $\eta=\gamma(\alpha, \varepsilon)$, defined on a small open neighbourhood of $\alpha=0, \xi=0$ which we may take of the form $J \times V$, where $J$ is an open interval of the real line containing $\alpha=0$, and $V$ an open set in $F^{c}$ containing $\varepsilon=0$. By definition, $r$ and its first partial derivatives vanish at $(0,0)$. According to the analysis of Section B.5, the motion on the local center manifold near $\alpha=0, x=0$, is governed by the difference equation, for $\left(\alpha_{n}, \xi_{n}\right)$ in $J \times V$,

$$
\begin{aligned}
& \alpha_{n+1}=\alpha_{n} \\
& \xi_{n+1}=C \varepsilon_{n}+f\left(\varepsilon_{n}, \gamma\left(\alpha_{n}, \xi_{n}\right), \alpha_{n}\right) \equiv \Gamma\left(\varepsilon_{n}, \alpha_{n}\right),
\end{aligned}
$$

with $n_{n}=\gamma\left(\alpha_{n}, \varepsilon_{n}\right)$ for a $11 n \geq 0$. In fact (Theorem B.5.3), the map $H$ is locally topologically conjugate to

$$
\alpha \rightarrow \alpha, \varepsilon \rightarrow \Gamma(E, \alpha), \eta+B \eta,(\alpha, E) \text { in } J \times V, \eta \text { in } F^{s}+F^{U} \text {. }
$$

Studying the local bifurcation of the family $G_{\alpha}$ in $R^{m}$ amounts to analysing the local bifurcation of the family $\Gamma_{\alpha}=\Gamma(, \alpha): V \rightarrow F^{c}$. It is possible to show that $\gamma(\alpha, 0)=0$, in which case $\Gamma(0, \alpha)=0$, for all $\alpha$ in $J$, see Iooss (1979, Chapter V, Section 3). One has moreover $D_{E} \Gamma(0,0)=C$, and $C$ is a matrix the eigenvalues of which have all modulus one. The dimension of the 
bifurcation problem is indeed reduced to the number of eigenvalues of the Jacobian matrix $D_{x} G(0, \alpha)$ that have modulus one at $\alpha=0$. In particular, if only a simple real eigenvalue, or only a pair of simple nonreal conjugate eigenvalues, have modulus one at $\alpha=0$, one may try and apply to the family $\Gamma_{\alpha}$, the bifurcation theorems presented in the preceding three sections, on the real line or in the plane. A precise representation in the ambient space $R^{m}$, of the bifurcating fixed points, periodic orbits or invariant closed curves, will then be obtained through the transformation $\xi \rightarrow(\xi, \gamma(\alpha, \xi))$, for each $\alpha$. Stability or unstability in $R^{m}$, in the dynamics generated by $G_{\alpha}$, will then be deduced from the fact, already mentioned, that $H$ is locally topologically conjugate to $\alpha+\alpha, \xi \rightarrow \Gamma(\xi, \alpha), \eta \rightarrow B n$. In particular, if all eigenvalues $\lambda$ of the matrix $B$ satisfy $|\lambda|<1$, and if an object $K$ in $F^{C}$ (fixed point, periodic orbit, etc ...) has been found to be asymptotically stable in the dynamics generated in $F^{C}$ by $\Gamma_{\alpha}$; the image of $K$ in $R^{m}$ after the transformation $\xi \rightarrow(\varepsilon, \gamma(\alpha, \xi))$, is also asymptotically stable in the dynamics generated in $R^{m}$ by $G_{\alpha}$.

Remark C.5.1. In practice, in order to apply the bifurcation theorems of the preceding sections, one needs only to know the partial derivatives of $\Gamma(E, \alpha)$, and thus of $\gamma(\alpha, \xi)$, at $\alpha=0, \varepsilon=0$. One can compute them by using the identification technique described in Remark B.5.4.

Remark C.5.2. We already noted that $\gamma(\alpha, 0)=0$ for all $\alpha$ in $J$. For every $\bar{\alpha}$, the graph in $R^{m}$ of $r(\bar{\alpha},$.$) represents the section of W_{(0,0)}^{c}$ in $R^{m+1}$ by the hyperplane of equation $\alpha=\bar{\alpha}$; it is therefore a local invariant manifold $W_{\bar{\alpha}}$ 
of $G_{\bar{\alpha}}$ near $(E, \eta)=0$, having the same dimension as $F^{c}$. Since $W_{(0,0)}^{c}$ is locally attracting in the dynamics generated in $R^{m+1}$ by the map $H$, the manifold $W_{\alpha}$ is also locally attracting in the dynamics generated in $R^{m}$ by $G_{\alpha}$, for each $\alpha$. Since by construction $D_{\xi} \gamma(0,0)=0, W_{0}$ is actualiy a local center manifold of $G_{0}$. Under some regularity conditions, it can be shown that for $\alpha \neq 0$, the manifold $W_{\alpha}$ is tangent to the generalized eigenspace of $D G_{\alpha}(0)$ corresponding to the eigenvalues that cross the unit circle at $\alpha=0$, see Iooss 11979, chap. V.3, Lemma 3).

The fact that local center manifolds need not be unique is not troublesome here. Any invariant closed set that bifurcates from the origin in $R^{m}$ for the family $G_{\alpha}$, such as fixed points, periodic orbits or invariant closed curves, must belong to $W_{\alpha}$ for each $\alpha$, and this no matter, which specific choice of $W_{(0,0)}^{c}$ was made. For a precise statement, see Iooss (1979, Chapter V.3, Lemma 4).

\section{Notes on the literature}

The presentation of the saddle node and flip bifurcations in Sections C.2 and C.3 is a direct transposition of Whitley (1983, Section 1.1). The material of Section C.4 on the Hopf bifurcation is adapted from Iooss (1979, chap. III), Whitley (1983, Sections 2.2, 2.3 and 2.4), Guckenheimer and Holmes (1983, chap. 3.5). Other useful references are Carr (1981, chap. 3.4), Chenciner (1983, 1987), Marsden and McCraken (1976, Section 6). The material on the center manifold reduction is taken from Iooss (1979, Chap. V), Marsden and McCraken (1976, Section 2), Carr (1981, chap. 2.8), Guckenheimer and Holmes (1983, chap. 3.2). 


\section{FOOTNOTES TO APPENDIX C}

(1) Local bifurcations associated with several or multiples eigenvalues crossing the unit circle are far more difficult to handle, and at any rate are "rare" (nongeneric).

(2) We could have assumed $G(0,0)=0$ instead of $G(0, \alpha)=0$ for all $\alpha$. The origin is then a fixed point of $G_{0}=G(\ldots, 0)$, but since $\frac{\partial G}{\partial x}(0,0) \neq 1$, the fixed point would persist for small $\alpha$, and one would go back to the formulation of the text by translating it to the origin, according to the procedure outlined in the last part of Remark C.1.1. By continuity, we are sure that all $G_{\alpha}^{2}$ are well defined on a small common open interval containing 0 for a small enough. Thus all conditions of Proposition C.2.3 are satisfied.

(4) We could have assumed only $G(0,0)=0$ instead of $G(0, \alpha)=0$ for all $\alpha$. For then, since $D_{x} G(0,0)$ has no eigenvalue equal to 1 , the fixed point would persist for small $\alpha$, and one would translate it to the origin according to the procedure outlined in Remark C.1.1.

(5) For any complex number $z=x+$ iy, the notation $\operatorname{Re}(z)$ stands for the real part of $z$, i.e. $\operatorname{Re}(z)=x$.

(6) What follows is adapted from Whit ley (1983, Sections 2.3 and 2.4). See also Iooss (1979, chap. III.2).

(7) Here again, this simply means that there is a persistent fixed point of $G_{\alpha}$, that has been translated to the origin by a suitable $\alpha$-dependent change of coordinates. 


\section{GLOBAL BIFURCATIONS AND CHAOS}

We were interested in the preceding appendix in local bifurcations of a family of difference equations $x_{n+1}=G_{\alpha}\left(x_{n}\right)$, i.e. qualitative changes of the corresponding trajectories that can be observed in every, however sma11, neighbourhood of a fixed point of the family, when the indexing parameter varies. Such qualitative changes may of course occur only in some neighbourhood of the stationary state, sometimes quite far away from it. In that case, the bifurcation is global. Quite complex phenomena may then obtain : periodic orbits or even "chaotic" trajectories that look random although they are generated by a purely deterministic system. Many of these "exotic" phenomena are still poorly understood. We shall content ourselves here to give a brief account of what happens in the case of one-dimensional nonlinear difference equations, and of so-called "homoclinic" bifurcations of diffeomorphisms of the plane. Some notes at the end of the appendix will permit the interested reader to pursue her/his own inquiry.

\section{D.1. Maps of an interval}

We consider the difference equation

$$
x_{n+1}=G\left(x_{n}\right)
$$

where $x$ is a real number. We are interested in trajectories generated by (D. 1 ) that do not diverge to infinity, so we shall assume that $G$ leaves invariant an interval $[a, b]$, and consider only the restriction of $G$ to that interval, i.e. $G:[a, b] \rightarrow[a, b]$. 
If $G$ is continuous, it has a fixed point in the interval. More generally, (D.1) may have solutions that are periodic, or display an irregular, "turbulent" behaviour. We say that $x$ is a periodic point of period $k \geq 1$, if $x$ is a fixed point of the $k$-th iterate of $G$, i.e. $x=G^{k}(x)$, and if $k$ is the smallest integer having this property. As usual, the iterates of $G$ are defined recursively through $G^{j}(x)=G\left(G^{j-1}(x)\right)$. The corresponding periodic orbit, or cycle, is then the set $\left\{x, G(x), \ldots, G^{k-1}(x)\right\}$ 。

\section{Existence of cycles}

Global conditions ensuring the existence of cycles are relatively easy to find and to verify in this simple context, when the period is small. We give an example for cycles of period two and three.

Lemma $D .1 .1$. Assume that $G:[a, b] \rightarrow[a, b]$ is continuous. Let $\bar{x}$ in $(a, b)$ be a fixed point. with $G(x)>\times$ for $a l l \times$ in $(a, \bar{x})$. If there exists $x_{0}$ in $(a, \bar{x})$ such that $G^{2}\left(x_{0}\right) \leq x_{0}$ (resp. $\left.G^{3}\left(x_{0}\right) \leq x_{0}\right)$, then $G$ has a cycle of period two (resp. three).

We give a short proof in the period two case. Let $x_{0}$ in $(a, \bar{x})$ be such that $G^{2}\left(x_{0}\right) \leq x_{0}$. Assume first that the end point a is not a fixed point of $G$. Since $G^{2}(a) \geqslant a$, by continuity, $G^{2}$ has a fixed point $x$ in $\left[a, x_{0}\right]$ that is not a fixed point of $G: x$ is a periodic point with period 2 . If on the other hand $G(a)=a$, one has $G^{2}\left(x_{1}\right)>x_{1}$ for $x_{1}$ close enough to $a$. Then, again by continuity, $G^{2}$ has a fixed point in $\left(x_{1}, x_{0}\right]$, which is a periodic point with period 2. The proof for the period three case follows the same line. The reader will easily verify that the argument works for any period $k$ that is prime. 
Fig. 0.1 represents the graph of a map $G$ that satisfies the conditions of the Lemma, and that has accordingly a cycle of period two and a cycle of period three. The example shows that we should generally expect cycles of different periods to coexist. The following result characterizes the way in which such a coexistence may obtain. It implies in particular that a continuous map from an interval into itself that has a cycle of period three, as in Fig. D. 1, has in fact cycles of every period, and can thus generate quite complex trajectories.

\section{Fia. D.1}

Theorem 0.1 .2 (Sarkovskii). Consider the following ordering of the integers

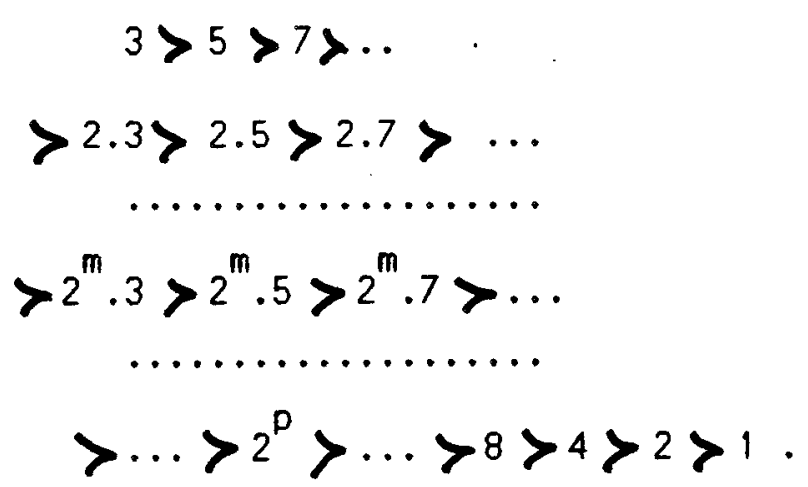

If the continuous map $G:[a, b]+[a, b]$ has a cycle of period $k$, then it has also a cycle of period $k^{\prime}$ for all $k^{\prime}<k$.

\section{Stability}

Finding which cycles are stable, if there are any, is an important task when one is faced with so many possible equilibria of the system. Stability and asymptotic stability were defined in appendix $B$ for a fixed point or for any closed set (Remark B.1.1). The definition applies therefore to a periodic 
85

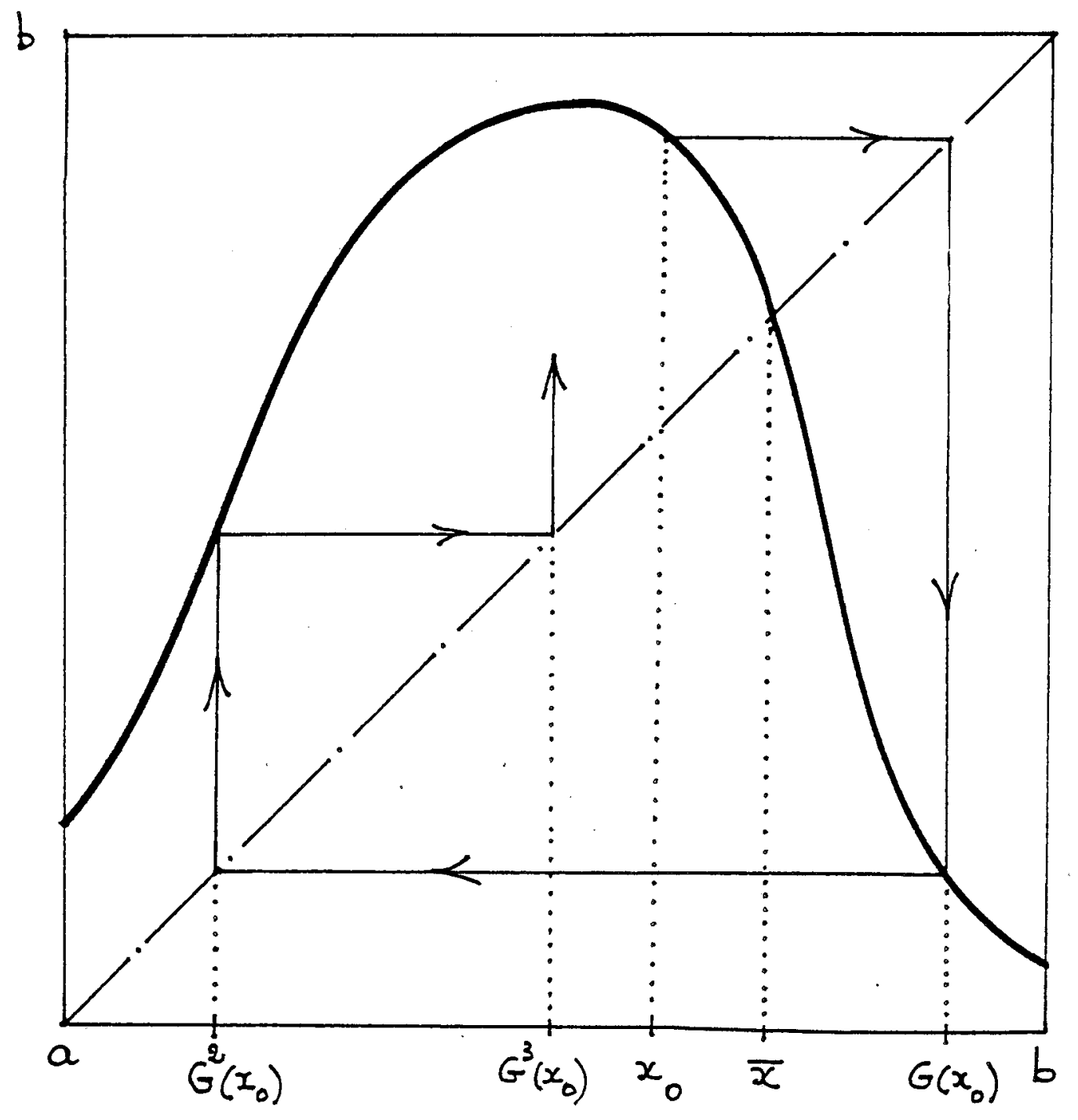

Fig.D.1 
orbit $\left(x_{0}, x_{1}, \ldots, x_{k-1}\right)$, where $G^{k}\left(x_{0}\right)=x_{0}$ and $G^{n}\left(x_{0}\right)=x_{n} \neq x_{0}$ for $n=1, \ldots, k-1$. As a matter of fact, since $x_{0}$ is a fixed point of $G^{k}$, (asymptotic) stability of the periodic orbit is equivalent to the (asymptotic) stability of $x_{0}$ in the difference equation associated to $G^{k}$ (Remark B.1.2). If $G$ is continuously differentiable, we have from the chain rule of differenciation

$$
\left(G^{k}\right) \cdot\left(x_{0}\right)=G^{\prime}\left(x_{k-1}\right) \ldots G^{\prime}\left(x_{0}\right)
$$

Thus if $a=\left(G^{k}\right)^{\prime}\left(x_{0}\right)$, the cycle is asymptotically stable when $|a|<1$, unstable when $|a|>1$. In the sequel it will be useful to say that the periodic orbit is weakly stable if $|a| \leqq 1$. This terminology is a slight abuse of language, since when $|a|=1$, the cycle may be stable or unstable depending upon the sign of higher order derivatives of $G^{k}$ at $x_{0}$, but it will turn out to be consistent in the cases under consideration. The cycle will be said superstable if $a=0$; in view of $(D .2)$, this means that a critical point of $G$, i.e. a point $x$ such that $G^{\prime}(x)=0$, belongs to the periodic orbit. Finally, one may remark that none of the foregoing definitions depends upon the particular point chosen on the periodic orbit. Indeed, we get, again from the chain rule of differenciation, for any element $x_{n}$ of the cycle

$$
\begin{aligned}
\left(G^{k}\right) \cdot\left(x_{n}\right) & =G^{\prime}\left(x_{n-1}\right) \ldots G^{\prime}\left(x_{0}\right) G^{\prime}\left(x_{k-1}\right) \ldots G^{\prime}\left(x_{n}\right) \\
& =\left(G^{k}\right) \cdot\left(x_{0}\right) .
\end{aligned}
$$

There is little hope to be able to say something on the stable periodic solutions of $(D .1)$ if one does not make simplyfing regularity assumptions about 
the map $G$, and its iterates. The first regularity assumption we shall make is that $G$ is single peaked.

(D.3) $G$ is $C^{\prime}$ and single peaked. i.e. there exists $x^{*}$ in $(a, b)$ such that $G^{\prime}\left(x^{*}\right)=0$, with $G^{\prime}(x)>0$ for $x<x^{*}$ and $G^{\prime}(x)<0$ for $x>x^{*}$. Moreover $G\left(x^{*}\right)=b$.

The assumption $G\left(x^{*}\right)=b$ involves no loss of generality whenever $G\left(x^{*}\right)>x^{*}$, since one can then restrict attention to the invariant interval $\left[a, G\left(x^{\star}\right)\right]$. The assumption implies that $G$ has a unique fixed point $\bar{x}$ in $\left(x^{*}, b\right)$. The next condition states that $G$ has no fixed point in the interval $\left(a, x^{\star}\right)$, and that if the end point a is a fixed point, it is unstable.

$$
G(x)>x \text { for } a 11 \times \text { in }\left(a, x^{*}\right) \text {, and } G^{\prime}(a)>1 \text { if } G(a)=a \text {. }
$$

The assumption that. $G$ is single peaked rules out multiple ups and downs in the graph of 6 . The curve representing this graph may still make a lot of "little waves" within each interval of monotony of $G$, and that is something we wish to avoid, not only for $G$ but also for the iterates $G^{n}$.

It turns out that the phenomenon is indeed prevented to occur if one assumes that $G$ has a negative Schwarzian derivative. This is a global version of a condition that we already met when studying the local bifurcations of maps of the real line : we saw that a flip bifurcation is supercritical if, and in general only if, the value of the Schwarzian derivative of the map, evaluated at the fixed point, was negative (Remark C.3.3). If we note the Schwarzian derivative (assuming that $G$ is of class $c^{3}$ ) 


$$
S G(x)=\frac{G^{\prime \prime}(x)}{G^{\prime}(x)}-\frac{3}{2}\left[\frac{G^{\prime \prime}(x)}{G^{\prime}(x)}\right]^{2},
$$

the condition reads

(D.5) $G$ is $C^{3}$ and $S G(x)<0$ for every $x$ such that $G^{\prime}(x) \neq 0$.

One can verify by direct computation that

$$
S G=-2\left|G^{\prime}\right|^{1 / 2}\left[\left|G^{\prime}\right|^{-1 / 2}\right] \cdots .
$$

So the assumption $(D .5)$ - which we will write for short $S G<0$ - means that $\left|G^{\prime}\right|^{-1 / 2}$ is convex on every interval of monotony of $G$. It will be satisfied in particular when $\left|G^{\prime}\right|$ or Log $\left|G^{\prime}\right|$ is concave on such intervals. One may note, incidentally, that the concavity of $G$ is neither necessary nor sufficient to guarantee $S G<0$.

The next fact implies that assumption ( 0.5$)$ rules indeed out "little waves" not only in the graph of $G$, but also in the graphs of all its iterates.

Lemma $0.1 .3 . \quad 1)$ If $S G<0$, then $1 G^{\prime} I$ has no positive local minimum.

2) For any two $C^{3}$ maps $G$ and $H$ from the real line into itself.

one has

$$
S(G \circ H)(x)=S G(H(x))\left[H^{\prime}(x)\right]^{2}+S H(x) \text {. }
$$

Thus $S G<0$ and $S H<0$ imply $S(G o H)<0$. In particular. $S G<0$ implies $S G^{n}<0$ for $211 n \geq 1$

It turns out that the assumption $S G<0$ achieves a lot more. Indeed, when $G$ is single peaked, i.e. satisfies assumptions $(D .3)$ and (D.4), there exists at most one weakly stable cycle, as the following theorem shows. To 
state the resuits most conveniently, it will be useful to say that a periodic orbit $P$ attracts a point $x$ if the set of limit points of the sequence of all iterates $G^{n}(x)$, as $n$ tends to $+\infty$, coincides with $P$.

Theorem D.1.4. Assume that $G:[a, b]$ satisfies $(0.3),(0.4)$ and $(0.5)$. Then

1) the map $G$ has at most one weakly stable periodic orbit. This periodic orbit lies in the interval $[G(b), b]$.

2) If $G$ has a weakly stable periodic orbit, it attracts the critical point $x^{\star}$. If in addition

$$
G^{\prime \prime}\left(x^{\star}\right)<0
$$

the weak ly stable periodic orbit attracts all points of the interval $[a, b]$, except for a set of Lebesque measure 0 .

This result is remarkable for it shows that if we restrict attention to those maps that satisfy the regularity conditions (D.3) through $(D .6)$, we are sure that there exists at most one weakly stable periodic orbit, even though there may be an infinity of cycles. When such a stable periodic orbit exists, it attracts actually most points of the interval. The last feature yields a nice practical method to discover the stable cycle when it exists. It suffices indeed to choose at random a point in the interval, to iterate it by using a computer, and to check whether the iterates converge to a periodic trajectory. If they do, we are sure that the limit cycle is weakly stable (if it were not, one would not have converged to it, if only as a result of rounding off errors). Since iterations have to be stopped after a finite time in practice, this experimental method will enable us to discover cycles that have not too 
large a period, and that are attracting enough. Of course, this way of proceeding will now allow us distinguishing between the presence of a weakly stable cycle that has a very long period or that is very weakly attracting, and the absence of any stable cycle.

The foregoing theorem can also be used to construct maps satisfying (D.3) through $(0.5)$, that have no stable cycle: it suffices that the iterates of the critical point $x^{*}$ do not converge, or that they fall upon an unstable cycle. Fig. D.2.a and b give an example of such a pattern, in which the iterates of the critical point hit an unstable fixed point. The maps pictured in these figures satisfy the conditions of Lemma 0.1 .1 ; they have thus a cycle of period three, and therefore from Sarkovskil's theorem, cycles of every period. None of these cycles is stable, however, when the maps have a negative Schwarzian derivative. In such a case, for any initial condition that does not belong to a periodic orbit, the corresponding trajectory will be aperiodic no matter how long one waits. Maps in this category are good candidates to portray "turbulent" or "chaotic" behaviour. We shall go back to such maps later on when dealing specifically with that issue.

\section{Fig. D.2.a Fig. D.2.b}

Remark D.1.5. It is not difficult to verify that assumptions (D.3), (D.4) and (D.6) are preserved when making a change of variable of the form $x \rightarrow h(x)$, where $h$ is a $c^{r}$ diffeomorphism that preserves orientation, i.e. such that $h^{\prime}(x)>0$ for all $x$. By contrast, the condition $S G<0$, as any global convexity assumption, is not preserved through a noninear change of variable. In practice, one may get a map $F$ that represents "naturally" a particular physical 


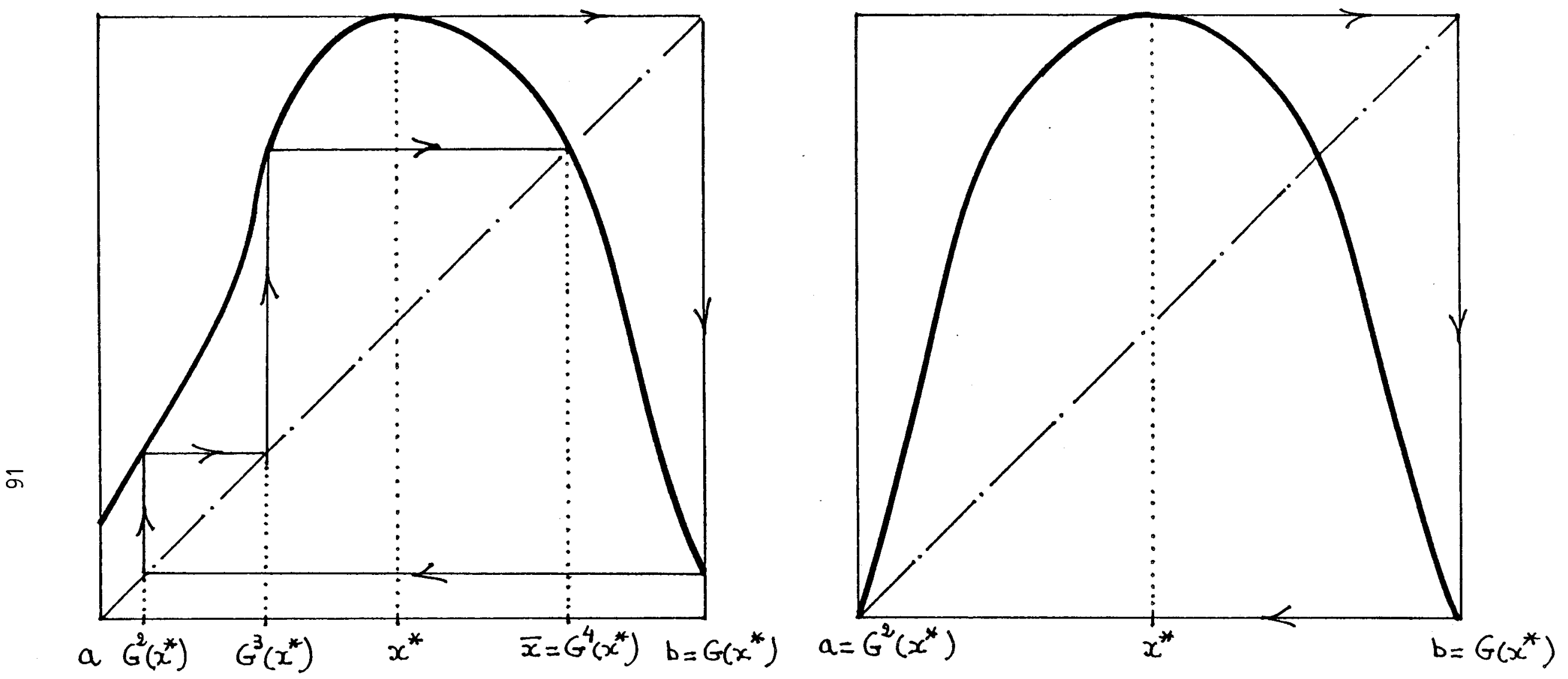

Fig.D.2.a

Fig.D.2.6 
or social phenomenon, and that does not have a negative Schwarzian derivative. Theorem 0.1 .4 applies nevertheless if one of the topological conjugates of $F$, say $G$, does satisfy $S G<0$. That remark applies of course also to the results be low that use the negative Schwarzian derivative condition.

\section{Bifurcations}

Consider a family of maps $G_{\alpha}$ indexed by the real number $\alpha$. When the parameter varies, the map $G_{\alpha}$, or one of its iterates $G_{\alpha}^{n}$, may undergo a bifurcation. Since we are considering here maps of the real line, the bifurcation may be of the saddle node type (Section C.2), or a flip bifurcation (Section C.3). Moreover, if each element $G_{\alpha}$ of the family is assumed to have a negative Schwarzian derivative, any iterate $G_{\alpha}^{n}$ has the same property, and every such flip bifurcation should be supercritical, a stable cycle of period $k$ becoming unstable and giving rise to a stable cycle of period $2 k$ (Remark C.3.3). When dealing with such families, one should expect to observe a cascade of period doubling bifurcations, involving stable cycles, as the parameter varies. This is indeed the pattern one observes in numerical experiments. We review here some mathematical results that explain the phenomenon.

Consider a family of maps $G_{\alpha}$ from $\left[a,{ }_{\alpha}, b_{\alpha}\right]$ into itself, in which the parameter $\alpha$ belongs to $[0,1]$. We assume that each $G_{\alpha}$ is single peaked, i.e. satisfies assumption (D.3) with a unique critical point $x_{\alpha}{ }_{\alpha}$, and moreover, that $a_{\alpha}$ and $b_{\alpha}$ depend continuously on $\alpha$, as well as $G_{\alpha}$ and its first derivative. We shall say that the family is full if

1) for $\alpha=0$, one has $G_{0}^{2}\left(b_{0}\right)>x_{0}^{*}$. In that case all iterates of the critical point, i.e. $G_{0}^{n}\left(x_{0}^{*}\right)$, belong to the interval $\left[G_{0}\left(b_{0}\right), b_{0}\right]$; 
2) for $\alpha=1$, one has $G_{1}^{2}\left(x_{1}^{*}\right)<x_{1}^{*}$ and $G_{1}^{3}\left(x_{1}^{*}\right)<x_{1}^{*}$.

Thus in a full family, for $\alpha$ near 0 , the only periodic orbits that $G$ may have, are fixed points or cycles of period 2, as one can easily verify. By contrast, for $\alpha$ near 1 , the map $G_{\alpha}$ has a cycle of period three, in view of Lemma D.1.1, and thus from Sarkovskii's theorem, cycles of every period.

Although Sarkovskii's theorem is not a statement about stable cycles, one should expect nevertheless that, in a full family, for any period $k$, a stable cycle of that period should appear for some open interval of the parameter, somewhere in $[0,1]$. Furthermore, in view of the ordering of the integers appearing in Theorem D.1.2, stable cycles of a period equal to a power of 2 should appear first, i.e. for smaller values of $\alpha$. The next fact makes precise this intuition.

Theorem D.1.6. Consider a continuous, full, one-parameter family of single peaked maps $G$ indexed by $\alpha$ in $[0,1]$, as above. Then

1) given any $k \geq 2$, the set of parameters $\alpha$ for which $G$ has a superstable cycle of period $k$ is non-empty and closed. Given such an $\alpha$, there is an open interval containing a such that $G_{\beta}$ has a stable cycle of period $k$ for every 8 in this interval.

2) Let $\alpha_{p}^{*}$ be the first value of $\alpha$ for which there exists a superstable cycle of period $2^{p}$ for $p \geq 1$. Then the sequence $\alpha_{p}^{*}$ increases with $p$ and converges to some value $\alpha_{\infty}^{\star}<1$ as $p$ tends to $+\infty$. For each $\alpha$ in $\left[0, \alpha_{\infty}^{\star}\right)$, a 1$]$ cycles of the map $G$ have a period that is a power of 2 or are fixed points. 
3) If superstable cycles of periods $2^{p}$ and $2^{q}$ with $q>p+1$ occur respective $1 y$ for the values $\alpha$ and $\beta$ in $\left(0, \alpha^{*}\right)$, then a superstable cycle of period $2^{k}$, with $a>k>p$, must appear for some value in the open interval defined by $\alpha$ and $\beta$.

The foregoing result is valid even for maps that have not a negative Schwarzian derivative. If we make that additional assumption, we get

Theorem 0.1 .7 . Consider a continuous, full, one-parameter family of maps $G_{\alpha}$ that satisfy each $(D .3),(0.4),(0.5)$ and $(0.6)$. Then

1) for any $\alpha$ in $\left(0, \alpha^{*}\right)$, the map $G_{\alpha}$ has a unique weakly stable cycle ;

2) there is an uncountable set of values of $\alpha$ in $\left(\alpha_{\infty}^{*}, 1\right]$ for which $G$ has no weak 7y stable periodic orbit.

These results can be translated into a (global) bifurcation diagram, in the spirit of the (local) bifurcation diagrams that were used in the previous appendix, by putting on an horizontal axis the parameter $\alpha$, and by representing on a vertical axis, for each value of $\alpha$, the corresponding stable periodic orbit(s), whenever it exists. If we focus on the case where each member of the family has a negative Schwarzian derivative, we obtain a cascade of global period doubling bifurcations, as in Fig. 0.3 , on the interval $\left[0, \alpha_{\infty}^{*}\right)$. There, stable periods equal to a power of 2 are "visited" consecutively as in the last line of Sarkovskii's ordering of the integers (but not necessarily monotonically : think of a nonmonotonic reparametrization of the family). Stable cycles with a period different from a power of 2 must (and will) occur 
in open intervals of $\left(\alpha_{\infty}^{*}, 1\right]$. Once such a stable period obtain, say an odd number, one should observe subsequently a cascade of global period doubling bifurcations. For an uncountable number of values in $\left(\alpha_{\infty}^{*}, 1\right]$, the map is "aperiodic" : there is no stable cycle.

\section{Fig. 0.3}

Such a pattern can be produced "experimentally", by using Theorem D.1.4, and simulating the difference equations on a computer. One may employ a grid of values of the parameter, and plot on a vertical axis above each $\alpha$ in the grid, the values of the iterates $G_{\alpha}^{n}(x)$, say for $n=100$ to $n=200$, of a point chosen at random in the corresponding interval $\left[a_{\alpha}, b_{\alpha}\right]$. If each $G_{\alpha}$ has a negative Schwarzian derivative, this procedure will display clearly stable cycles that have a low period and are attracting enough. The experimental procedure will thus reproduce neatly the cascade of period doubling bifurcations in $\left[0, \alpha_{\infty}^{\star}\right)$. Beyond $\alpha_{\infty}^{\star}$, we shall observe typically in some places, little windows with small stable periods such as 3 or 5 , together with the beginning of the corresponding period doubling cascade; in other places, we will observe a "mess" : there is then either no stable cycle, or a stable cycle with a long period or that is only little attracting.

Fig. D.4 describes such an experimental bifurcation diagram for the logistic family

$$
G_{\alpha}(x)=1-\alpha x^{2}, x \text { in }[-1,1]
$$


96

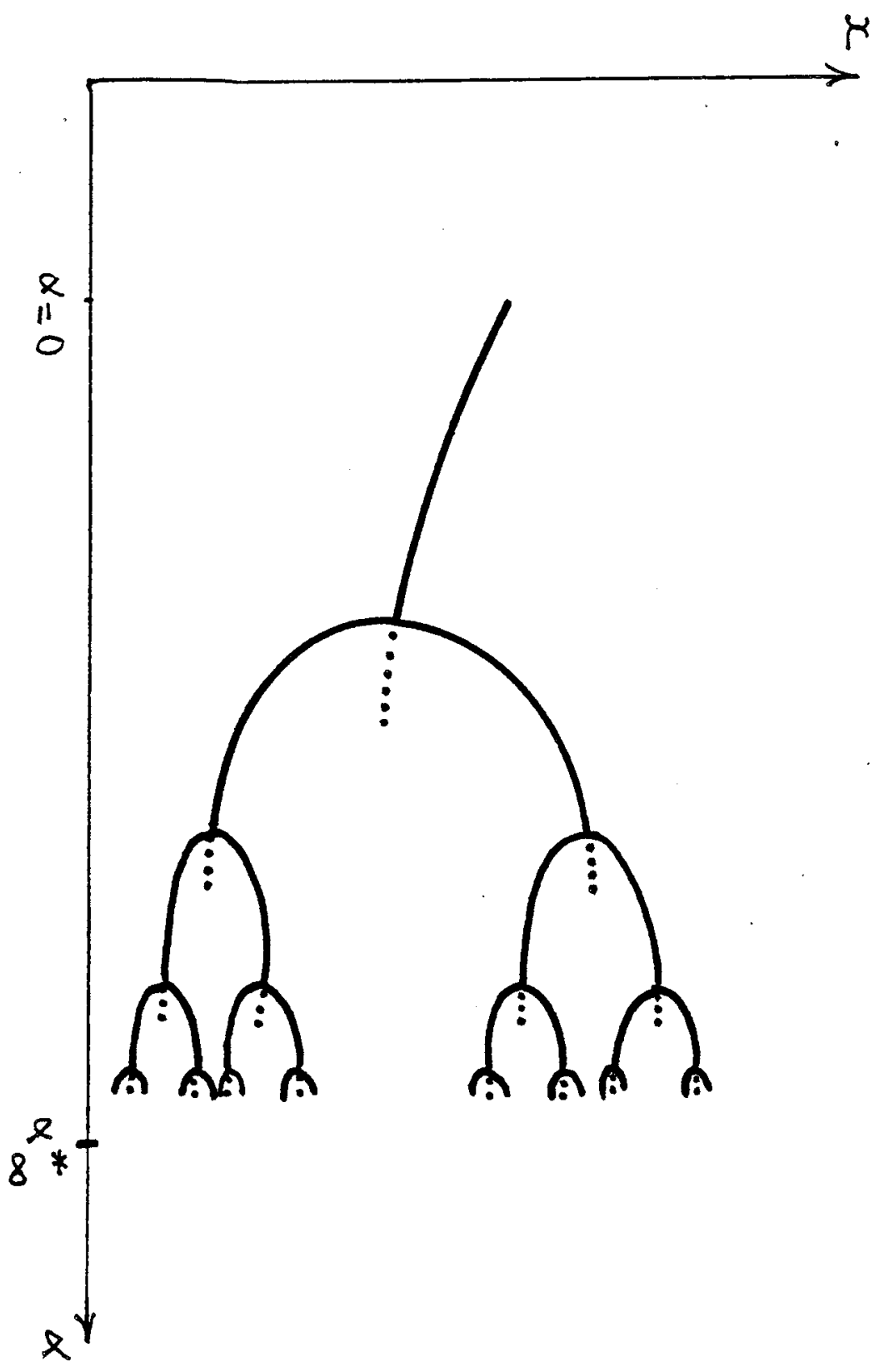


for $0<\alpha \leqq 2$. For each value of the parameter $\alpha$ in that interval, $G_{\alpha}$ is single peaked, with a unique critical point at $x^{\star}=0$ and $G_{\alpha}\left(x^{*}\right)=1>x^{*}$; it has a negative Schwarzian derivative, since $G_{\alpha}^{\prime \prime}(x) \equiv 0$. It is easy to verify that $G^{2}\left(x^{*}\right)>x^{*}$, for $\alpha$ close to 0 . For $\alpha=2$, one has $G_{\alpha}^{2}\left(x^{*}\right)=G_{\alpha}^{3}\left(x^{*}\right)=-1<\stackrel{*}{x}$; the graph of $G_{\alpha}$ is then as in Fig. D.2.b : there is a cycle of period three, hence a cycle of every period, and none of these cycles is stable. The logistic family, for $\alpha$ in $(0,2]$, is full.

\section{Fig. D.4}

\section{Deterministic chaos}

Nonlinear difference equations on the real line can generate periodic orbits, as we have seen. They can also yield much more complex trajectories, that look "random" and rather unpredictable, although they are generated by a purely deterministic dynamical system. We have already alluded to the possibility of such a behaviour when giving examples of maps that have no stable cycle (Fig. D.2). The phenomenon has attracted a lot of attention, for it seems to open the possibility to "explain" phenomena that look apparently random by deterministic nonlinear dynamical systems, and thus to reconcile 19th century determinism and unpredictability.

The notion of chaos that we wish to discuss first is due to $L i$ and Yorke. We say that the map $G$ from $[a, b]$ into itself is chaotic if there is an uncountable subset $S$ of the interval, containing no periodic orbits, such that

1) for every $x, y$ in $S, x \neq y$,

$$
\begin{aligned}
& \lim \sup _{n}\left|G^{n}(x)-G^{n}(y)\right|>0 ; \\
& \lim \inf _{n}\left|G^{n}(x)-G^{n}(y)\right|=0 ;
\end{aligned}
$$




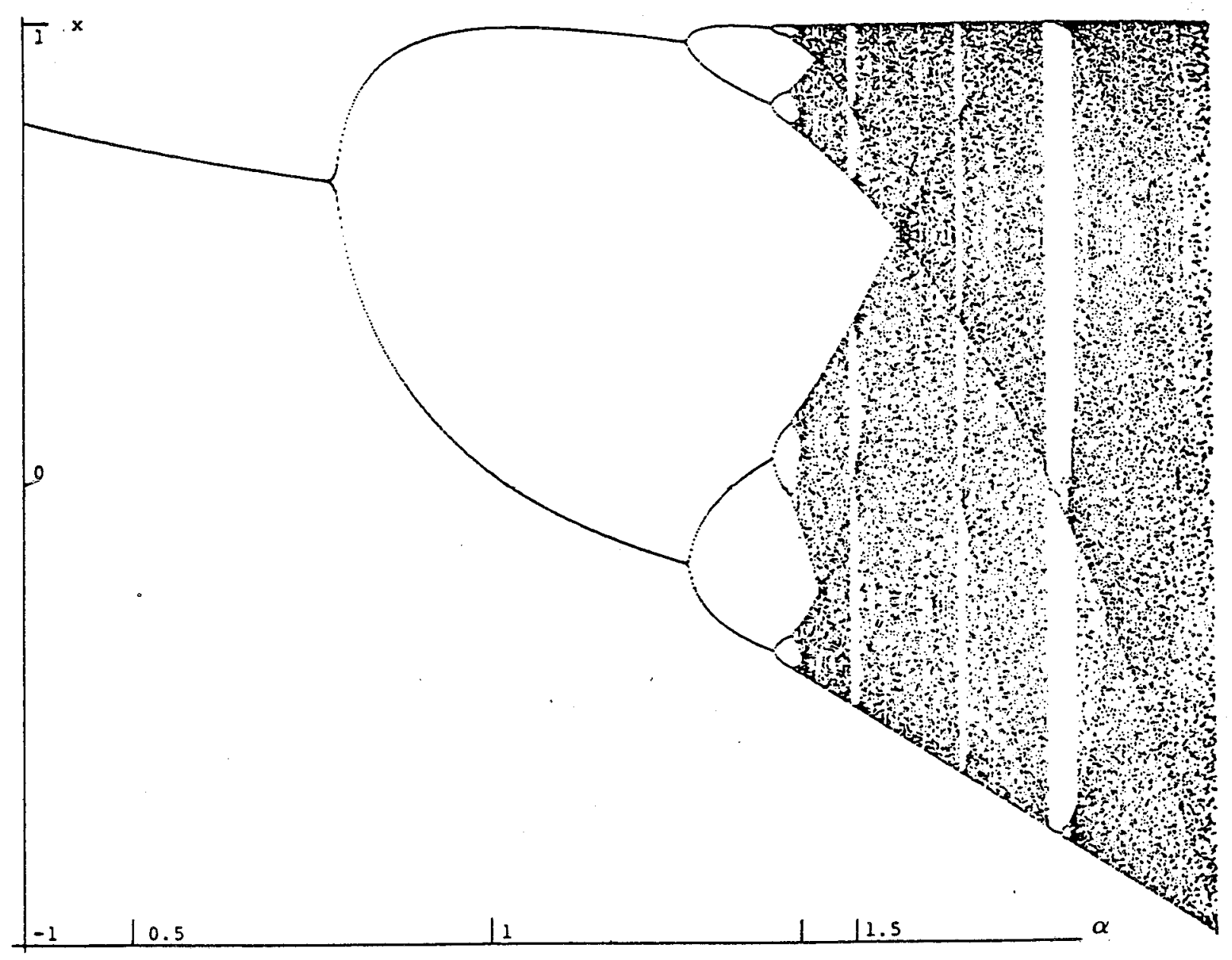

Fig D. 4 
2) for every $x$ in $S$ and $y$ periodic, $\lim \sup _{n}\left|G^{n}(x)-G^{n}(y)\right|>0$.

This definition means that for any two (different) initial conditions $x$ and $y$ in the chaotic set $S$, the corresponding trajectories become infinitely often extremely close to each other and also infinitely often noticeably separated. The notion seems to account for one essential feature of "turbulence" : small perturbations of initial conditions lead to trajectories that are notably different.

Li and Yorke (1975) proved that if the continuous map $G$ has a cycle of period three, then $G$ is indeed chaotic. On the other hand, it is not difficult to see that if an iterate $G^{n}$ is chaotic for some $n \geq 1$, then $G$ is also chaotic. If one puts together these facts with Sarkovskii's Theorem, one gets

Theorem 0.1 .8 . If the continuous map $G:[a, b] \rightarrow[a, b]$ has a cycle of a period that is not a power of two. then $G$ is chaotic.

This notion of chaos is not quite satisfactory from a "physical" point of view, however. It happens very often that although a given map $G$ is chaotic in the above sense, there is a stable periodic orbit that attracts most points of the interval with respect to the Lebesgue measure. In view of Theorem D.1.4, this will happen if $G$ is single peaked, has a negative Schwarzian derivative, and has a stable cycle of a period that is not a power of two. In a bifurcating family of maps $G_{\alpha}$ that are single peaked and have a negative Schwarzian derivative as in Theorems 0.1 .6 and 7 , maps $G_{\alpha}$ will often be chaotic in the sense of $L i$ and Yorke in the whole interval $\left(\alpha_{\infty}^{*}, 1 \mathrm{~J}\right.$, yet for many open 
subintervals, there will be a stable cycle attracting most points of $\left[a_{\alpha}, b_{\alpha}\right]$. In such circumstances, the presence of the chaotic set $S$ may affect the trajectories for some time, but will not influence the asymptotic behaviour of the system.

This discussion shows that in this context, a satisfactory notion of chaos should involve maps that have no stable cycle. In view of Theorem D.1.4, if $G$ is single peaked and satisfies $S G<0$, this means that the iterates of the critical point either do not converge, or hit an unstable perfodic orbit. Among the class of such aperiodic maps, of special interest are those which have an "invariant probability measure" that has an integrable density with respect to the Lebesgue measure. The probability measure $v$ on $[a, b]$ (endowed with its Borel o-algebra) is said to be invariant with respect to $G$ if $v\left(G^{-1}(A)\right)=v(A)$ for any Borel set. An example of such an invariant probability measure is provided by considering a periodic orbit $\left\langle x_{0}, x_{1}, \ldots, x_{k-1}\right\rangle$ and the probability measure $v$ that assigns the weight $1 / k$ to each $x_{j}, j=0, \ldots, k-1$. More generally, the support of an invariant probability measure, i.e. the smallest closed set $\wedge$ that has probability one, is an invariant set : $G(x)$ is in $\wedge$ whenever $x$ belongs to $\wedge$. If $\vee$ has a density (we shall say then that $v$ is continuous), the invariant set contains an uncountable set of points, and has positive Lebesgue measure. Finally, $v$ is said to be ergodic if for every $v$-integrable real function $f$,

$$
\frac{1}{n} \sum_{j=1}^{n} f\left(G^{j-1}(x)\right) \rightarrow \int f d v
$$

as $n$ tends to $+\infty$, for $v$-almost every $x$. This implies in particular that if one considers for each $x$ and every $n$, the empirical distribution $v_{n}(x)$ that is generated by the iterates $G^{j}(x)$ for $j=0, \ldots, n-1$, which assigns the weight 
$1 / n$ to each $G^{j}(x)$, then the sequence $v_{n}(x)$ converges weak ly to $v$ for $v$-almost every $\times$ (see, e.g. Parthasarathy (1967, Theorem 9.1) for a proof of that fact, and for a definition of the weak convergence of probability measures). Thus if $G$ has an invariant probability measure $v$ that is continuous and ergodic, most of the trajectories starting in the support $\wedge$ of $\vee$ will stay there and will look quite complex since they will eventually fill up the entire support of the limit distribution $v$. In fact, most of these trajectories will be dense in $\wedge$. Yet these trajectories display strong statistical regularities, since empirical distributions and time averages become asymptotically fairly stable for $v$-almost every initial point.

It turns out that maps having a graph like those of Fig. D.2. do have an invariant, continuous, ergodic probability measure, as the next fact shows.

Theorem D.1.9. Assume that the map $G$ from $[a, b]$ into itself satisfies $(D .3)$, (D.5) and (D.6), that it has no weakly stable periodic orbit and that there exists an open neighbourhood $V$ of the critical point $x$ such that the iterates $G^{n}\left(x^{*}\right)$ do not belong to $V$, for $n \geqslant 1$. Then $G$ has a unique continuous invariant probability measure. It is ergodic.

The foregoing conditions are met if $G$ satisfies $(D .3),(D .4)$, (D.5) and (D.6), and if the iterates of the critical point hit an unstable periodic orbit.

Another notion of chaos, which is more directly inspired from the concept of $L i$ and Yorke, is the following one. A map $G$ from $[a, b]$ into itself is said to have sensitive dependence on initial conditions if there exists a 
subset S. of the interval, having positive Lebesque measure, such that for every $x, y$ in $S$ with $x \neq y$,

$$
\begin{aligned}
& \lim \sup _{n}\left|G^{n}(x)-G^{n}(y)\right|>0 \\
& \lim \inf _{n}\left|G^{n}(x)-G^{n}(y)\right|=0
\end{aligned}
$$

The difference with $L i$ and Yorke is that one requires here "turbulence" to occur on a set of initial conditions that is "thick" enough, i.e. that has positive Lebesgue measure. According to Theorem 0.1 .4 , if the map $G$ is single peaked and has a negative Schwarzian derivative, sensitive dependence on initial conditions can obtain only if there is no stable cycle.

The idea of sensitive dependence on initial conditions can also be captured by using the notion of so-called Lyapounov exponents. Let $x$ be a point in $[a, b]$, and let $x_{j}=G^{j}(x)$ be its iterates for $j \geqslant 0$, with the convention that $x_{0}=x$. The Lyapounov exponent of $G$ at $x$ is then defined as

$$
\lim _{n \rightarrow \infty} \frac{1}{n} \sum_{j=1}^{n} \log \left|G^{\prime}\left(x_{j-1}\right)\right|=\lim _{n \rightarrow \infty} \frac{1}{n} \log \left|\left(G^{n-1}\right) \cdot(x)\right|
$$

whenever the limit exists. When the definition is specialised to the case of a fixed point, or more generally a periodic point of period $k$, the corresponding Lyapounov exponent is simply the logarithm of the modulus of $\left(G^{k}\right)^{\prime}(x)$. Intuitively, the Lyapounov exponent measures the average (exponential) rate of separation of the orbits of points near $x$ from the trajectory generated by $x$. Thus the idea that trajectories are sensitive to small changes of the initial condition $x$, may be expressed by the property that the Lyapounov exponent of $G$ at $x$ exists and is positive.

Consider now a map $G$ that has an ergodic, continuous invariant probability measure $v$. Then by definition of ergodicity, Lyapounov exponents 
exist for $v$-almost every $x$ in the support $\wedge$ of $v$; they are in fact equal to $\int \log \left|G^{\prime}(x)\right| d v$, and are thus independent of $x$. We may then speak of the Lyapounov exponent of $G$ on $\wedge$. If we assume that it is positive, then the set $\wedge$ has positive Lebesgue measure, and almost every initial condition $x$ in $\wedge$ gives rise to a trajectory that is dense in $\wedge$ and displays sensitive dependence on initial conditons. Although the law of motion of the system is deterministic, trajectories in $\wedge$ will look random, and will be in fact unpredictable after a significant number of iterations if one makes even small errors of measurement of the initial condition. The set $\Lambda$ is then an instance of what is called a strange attractor, and gives rise to deterministic turbulence or chaos.

A natural question arises in this context : what is the relative frequency of stable periodic behaviour and of chaos in one-dimensional difference equations? The issue involves in fact a subtle conflict between two notions of "genericity".

A convenient framework to analyze the issue is, again, to consider a one-parameter family of single peaked maps having a negative Schwarzian derivative, as in Theorem D.1.7. A common conjecture among mathematicians is that for many "reasonable" families in that class, the set of values of the parameter $\alpha$ for which a stable period obtains is open and dense in $[0,1]$. According to this topological viewpoint, maps $G_{\alpha}$ that have no stable cycles in the family are "rare", and moreover, structurally unstable, i.e. a small perturbation of the parameter $\alpha$ would lead back to a stable period. Examples of such structural unstability are most easily visualized by looking at Fig. D.2. The maps there have an invariant continuous probability measure, because the iterates of the critical point happen to hit upon an unstable fixed point. It 
is clear, however, that such a feature can be destroyed by a small perturbation of the map under consideration.

By contrast, it was shown by Jakobson (1981) that under the assumptions of Theorem D.1.7, for many "reasonable" families, the set of values of the parameter $\alpha$ for which $G_{\alpha}$ has an invariant, continuous ergodic probability measure, and displays sensitive dependence on initial conditions (has positive Lyapounov exponent) has positive Lebesque measure. According to this measure theoretic viewpoint, chaotic behaviour cannot be considered as rare. If one chooses at random the parameter $\alpha$ in the family, there is a positive probability to obtain a map that leads to deterministic chaos.

\section{D.2. Homoclinic bifurcations and horseshoes}

The example of complex periodic orbits and of chaos that were just described involved single peaked maps of the real line with a significant "hump". Such maps are admittedly special, but they contain a mechanism that seems to show up in many other cases of deterministic chaos that have been found in higher dimensions: if we look at the maps represented in Figure 0.2 , for instance, the image of the interval is obtained by stretching it, and folding it back into itself. We present now a diffeomorphism of the plane, the socalled horseshoe map, that is due to Smale, and leads to a complex invariant set through a similar mechanism. As we shall see, horseshoes arise for diffeomorphisms of the plane when there is a transverse crossing of the stable and ustable manifolds of a hyperbolic fixed point. The phenomenon was known, as least qualitatively, to Poincaré and Birkhoff. 


\section{The Horseshoe}

Consider a $C^{\prime}$ diffeomorphism $G$ of the plane, that sends the unit square $S$ onto the horseshoe shaped set $G(S)$, as in Figure $D .5$, with $A^{\prime}$ being the image of $A, B^{\prime}$ that of $B$, etc. (the map may actually be defined only on a neighborhood of the region that interests us). The transformation involves first a uniform vertical contraction by a factor $a<1$, second a uniform horizontal expansion by a factor $b>1$, and finally, a folding of the resulting rectangle in its middle. The only nonlinear transformation is the third one, and it is assumed that it concerns only points of the horseshoe that fall outside the unique square.

\section{Figure 0.5}

We are interested in characterizing the set $\wedge$ of points $x$ of the unit square such that $G^{n}(x)$ is in $S$ for a $11 n$, positive and negative. In this respect, it is useful to let $\Delta_{0}$ and $\Delta_{1}$ stand for the pre-images of the intersection of $G(S)$ and $S$, as in Figure D.6.a. By construction, the set $G(S) \cap S$ is composed of two horizontal rectangles of height a, while $\Delta_{0}$ and $\Delta_{1}$ are vertical rectangles of width $b^{-1}$.

The set of points $x$ of the unit square such that $G(x)$ belongs to $S$, is thus equal to the union of $\Delta_{0}$ and $\Delta_{1}$, or equivalently to $G^{-1}(G(S) \cap S)$. By iterating the map twice, we see that the image by $G$ of $G(S) \cap S$ is composed of two thinner horseshoes that are contained in $G(S)$. Their intersections with the unit square yield four horizontal rectangles of height $a^{2}$, the union of which form the set 

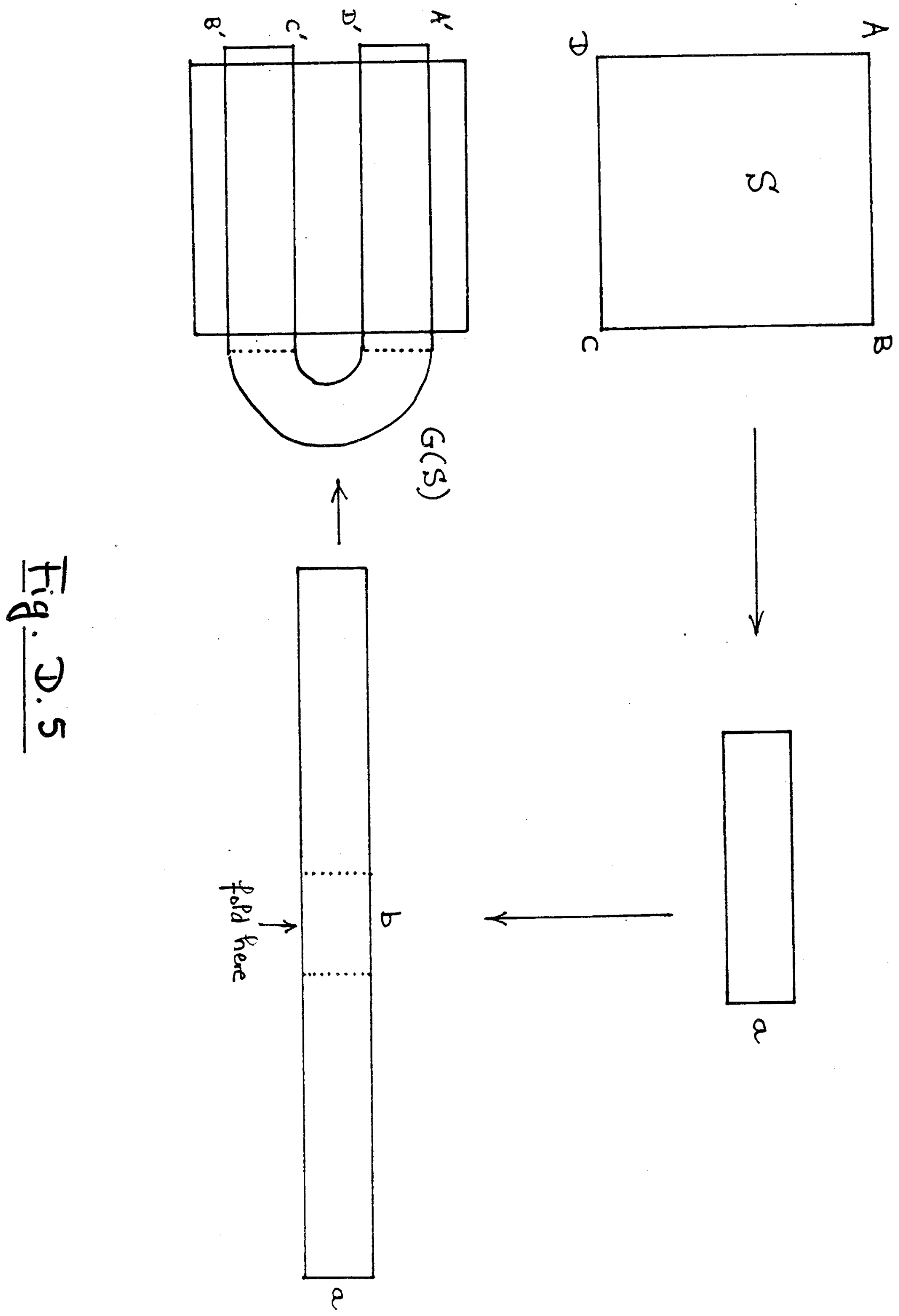


$$
G(G(S) \cap S) \cap S=G^{2}(S) \cap G(S) \cap S \text {, }
$$

see Figure D.6.b. The set of points $x$ of the unit square such that both $G(x)$ and $G^{2}(x)$ belong to $S$ is the union of the images by $G^{-2}$ of these four horizontal rectangles. This set is therefore composed of four vertical rectangles of width $b^{-2}$, as shown in Figure D.6.C. Proceeding by induction on $n$, one sees that the set of points $x$ of $S$ such that $G^{j}(x)$ is in $S$ for every $j=1, \ldots, n$, is the union of $2^{n}$ vertical rectangles of width $b^{-n}$, given by

$$
G^{-n}\left(G^{n}(S) \cap \ldots \cap G(S) \cap S\right)
$$

In the limit for $n++\infty$, the iterates $G^{j}(x)$ of $x$ lie in the unit square for all $j>0$ if and only if $x$ belongs to a family of vertical segments of $S$. The foregoing construction makes apparent that the projection of this family of vertical segments on the horizontal basis of $S$ is a Cantor set (also called a perfect set) $\Lambda_{+}$, i.e. a closed set such that 1) the largest connected subset is a point, and 2) every point of $\Lambda_{+}$is an accumulation point of $\Lambda_{+}$.

\section{Figure $0.6 .0 \quad$ Fiqure $0.6 .6 \quad$ Fiqure $0.6 . \mathrm{C}$}

One can proceed in a similar fashion for backward iterates. The set of points $x$ of $S$ such that $G^{-1}(x)$ is also on $S$ is equal to the intersection of $G(S)$ and $S$, i.e. to the two horizontal rectangles of Figure D.6.a. Similarly, the set of points $x$ of the unit square having the property that both $G^{-1}(x)$ and $G^{-2}(x)$ are in $S$, is composed of the four horizontal rectangles of Figure D.6.b. Proceeding by induction on $n$ and going to the limit, yields that the set of points $x$ of $S$ such that $G^{-n}(x)$ is in $S$ for every $n \geqslant 0$ is a family of horizontal segments. Its projection on the vertical side of the unit square is, 
$\mid$

$\mid \begin{aligned} & \theta \\ & \alpha \\ & \dot{\alpha}\end{aligned}$

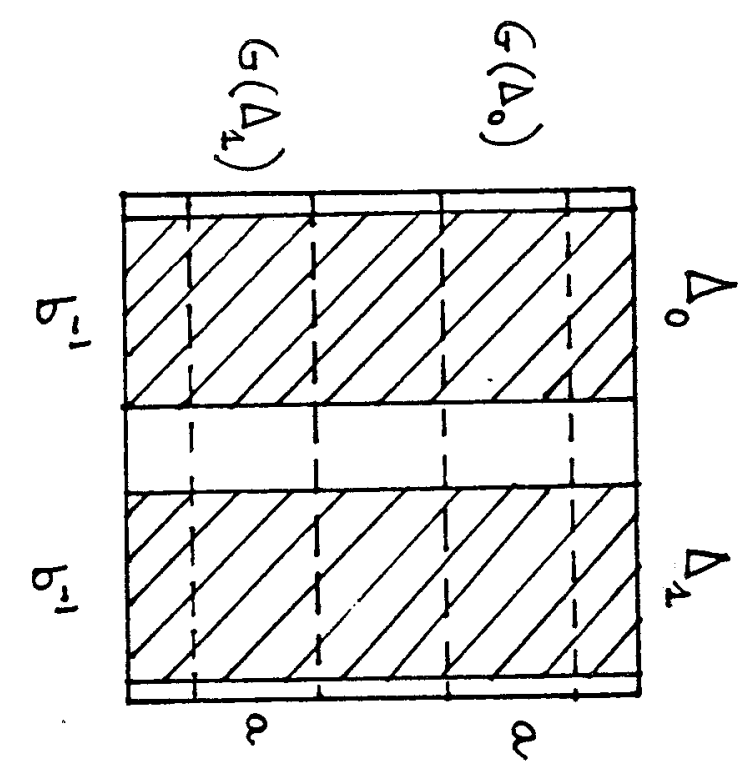

$\mid \begin{aligned} & \mid+0 \\ & 0 \\ & 0 \\ & 0 \\ & 0\end{aligned}$

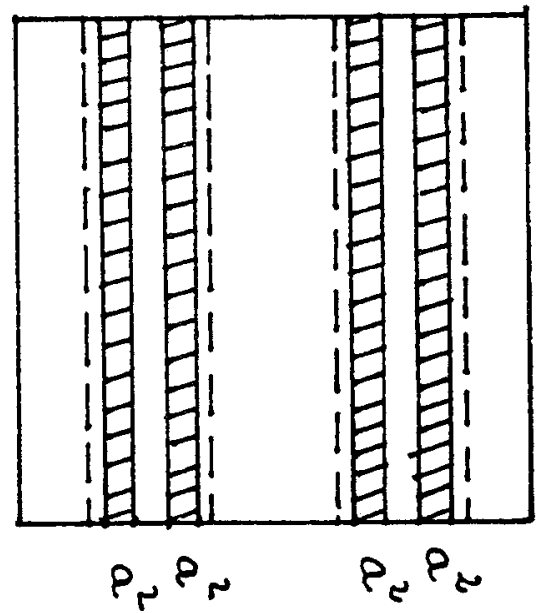

$1 j$
0
$\dot{\sigma}$
$\dot{\sigma}$
$\dot{n}$

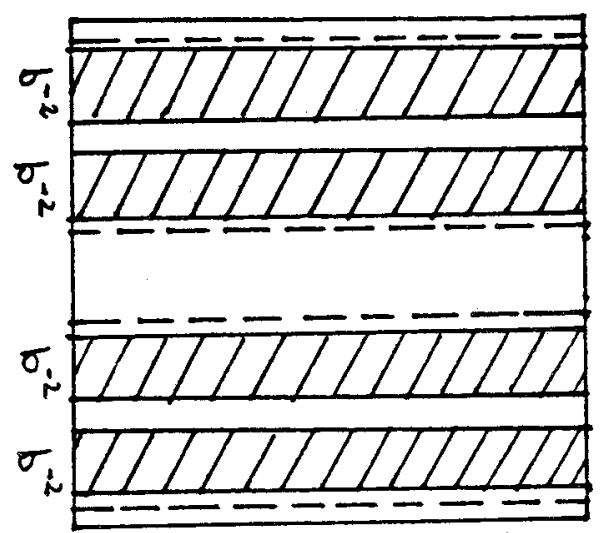


again, a Cantor set $\Lambda$.

Therefore $G^{n}(x)$ belongs to $S$ for all $n$, positive and negative, if and only if $x$ belongs to the product of these two Cantor sets, i.e. $\wedge^{\prime} \Lambda_{+} \times \Lambda_{-}$.

To describe the structure of the orbits that lie in the invariant set $\wedge$ that we just obtained, it is convenient to employ a method called symbolic dynamics. The principle of the approach is to substitute to the complex task of tracking down where the forward and backward orbits of a given point lie exactly, the much simpler task of identifying regions in which the points of the orbit may fall.

In the case at hand, if $x$ is in $\wedge$, every iterate $G^{n}(x)$ belongs either to $\Delta_{0}$ or $\Delta_{1}$, for each positive or negative $n$. For any $x$ in $\wedge$, we can therefore define a bi-infinite sequence $I(x)$, the $n$-th element of the sequence, i.e. $I_{n}(x)$, being equal to 0 if $G^{n}(x)$ is in $\Delta_{0}$, and to 1 if $G^{n}(x)$ belongs to $\Delta_{1}$, for $-\infty<n<+\infty$.

It can be shown that the map $x \rightarrow I(x)$ from $\wedge$ to the set $[$ of bi-infinite sequences of $0^{\prime} s$ and $1^{\prime} s$, is onto and one-to-one. In fact, a much stronger statement is true. Suppose that the set $[$ is endowed with the metric defined by

$$
d(I, J)=\sum_{-\infty}^{+\infty} \delta 2^{-|n|},
$$

with $\delta_{n}=0$ if $I_{n}=J_{n}$, and $\delta_{n}=1$ otherwise. Two sequences $I$ and $J$ are thus close in the topology induced by the metric (0.7), if and only if they agree on a long enough "central block", i.e. $I_{n}=J_{n}$ for all $|n| \leqslant N$ for some large enough $N$. Then the map $x \rightarrow I(x)$ from $\wedge$ onto $[$, endowed with this metric, is actually an homeomorphism. Since for any element $x$ of $\wedge$, the sequence 
corresponding to $G(x)$, i.e. $I(G(x))$, is obtained from the sequence $I(x)$ by shifting indices one place to the left, the restriction of $G$ to the invariant set $\wedge$ is topologically conjugate to the (left) shift automorphism $0:[\rightarrow[$ defined by $[\sigma(I)]_{n}=I_{n+1}$, for $-\infty<n<+\infty$ (see Guckenheimer and Holmes (1983, Theorem 5.1.1)).

This result enables us to substitute to the study of the orbits of points $x$ of $\wedge$, the analys is of the corresponding sequences $I(x)$. For instance, an element $x$ of $\Lambda$ is a periodic point, of period $k$, if and only if $I(x)$ is periodic, of period $k$. It follows that $G$ has two fixed points in $S$, one in $\Delta$ and the other in $\Delta$. More generally, $G$ has periodic orbits of every period in $\wedge$. It is not difficult to see that the set of these periodic orbits is actually dense in $\Lambda$. Indeed, let $x$ be in $\Lambda$ and consider the corresponding sequence $I(x)$. Let $J$ be a sequence that agrees with $I(x)$ for all $|n| \leqslant N$, and that has period $k>N$. By taking $N$ (and thus $k$ ) large enough, the sequence $J$ can be made as close as one wishes to $I(x)$ in $[$, endowed with the metric (D.7). Since the map $x \rightarrow I(x)$ is an homeomorphism, the olement $y$ of $\wedge$ such that $J=I(y)$, is a periodic point, with period $k$, and is also as close as one wishes to $x$.

The same type of argument shows that $\wedge$ contains an uncountable set of nonperiodic orbits, that is dense in $\wedge$. It is also quite easy to show that $\wedge$ contains a dense orbit. Indeed, let $J$ be a sequence in $\Sigma$ that has a subsequence every finite sequence of $0^{\prime} s$ and $I^{\prime}$ 's one can think of. By adapting the above density argument, the orbit generated by the element $y$ of $\wedge$ such that $I(y)=J$ is actually dense in $\wedge$. To sum up this discussion, 
Theorem D.2.1 (Smale) : The horseshoe map $G$ has an invariant Cantor set $\wedge$ such that

1) $\wedge$ has periodic orbits of every period. The set of such periodic orbits is dense.

2) $\wedge$ has an uncountable set of nonoeriodic orbits. This set is also dense in $\wedge$.

3) $\wedge$ has a dense orbit.

It is intuitively clear from the preceding discussion, (but nontrivial to prove) that the qualitative features of the example should be structurally stable, i.e. should remain valid after a small enough $c$ perturbation. Indeed, Theorem 0.2 .2 (Smale) : Let $G^{*}$ be a diffeomorphism of the plane that is $C^{\prime}$ close enough to the horseshoe map $G$ (at least in the region of interest). Then $G^{*}$ has an invariant Cantor set $\wedge^{*}$ such that the restriction of $G^{*}$ to $\Lambda^{*}$ is topologically conjugate to the restriction of $G$ to $\wedge$.

Remark D.2.3 : Despite its many attractive features, the invariant set $\wedge$ of the horseshoe map is not an attractor: if one perturbates slightly the initial condition $x$ so as to make it to fall outside $\wedge$, the forward or backward orbit of $x$ will eventually leave the set $S$. Yet, the presence of the complicated invariant set $\wedge$ will affect the behaviour of nearby trajectories, often for quite some time. For an illuminating example of a complicated invariant set that can be analyzed through the above symbolic dynamics methods, and that is indeed an attractor (the so-called solenoid), see Lanford (1983). The example is three dimensional, and involves stretching and folding a solid torus. 


\section{Homoclinic Bifurcations}

The qualitative features of the horseshoe map can be generated in the case of diffeomorphisms of the plane, when there is transverse crossing of the stable and unstable manifolds of a hyperbolic fixed point.

To see this point, consider a $C^{r}$ diffeomorphism $G$ of the plane, with $r \geqslant 1$, and let $\bar{x}$ be fixed point of $G$ (here again $G$ may be defined only on a neighborhood of $\bar{x}$ that is sufficiently large to make the following arguments to go through). We assume $\bar{x}$ to be hyperbolic, i.e. the Jacobian matrix $D G(\bar{x})$ has no eigenvalue of modulus one. We know that the local stable and ustable manifolds $W_{(\bar{x})}^{s}$ and $W_{(\bar{x})}^{u}$ are then well defined and unique in a sufficiently small neighborhood of the fixed point (Theorem B.5.1). The global stable manifold of $\bar{x}$ is then defined by taking the union of the backward iterates of $W^{s}(\bar{x})$.

$$
W^{s}=\bigcup_{n \geqslant 0} G^{-n}\left(W_{(\bar{x})}^{s}\right)
$$

Similarly, the global unstable manifold of $\bar{x}$ is obtained by taking the union of the forward iterates of $w_{(\bar{x})}^{u}$,

$$
W^{u}=\bigcup_{n \geqslant 0} G^{n}\left(W_{(\bar{x})}^{s}\right) \text {. }
$$

We consider the case where the global stable and unstable manifolds of $\bar{x}$ intersect transversely, as in Figure D.7.a. The point of intersection, say $y$, is then an homoclinic point. There must be actually infinitely many such homoclinic points : all forward and backward iterates of $y$, i.e. $G^{n}(y)$ for $-\infty<n<+\infty$, belong to both $W^{S}$ and $W^{u}$. The (forward and backward) orbit of $y$ 
is then called a transversal homoclinic orbit. Since $G^{n}(y) \rightarrow \bar{x}$ as $n \rightarrow+\infty$, the unstable manifold $W^{u}$ must cross more and more often the stable manifold $W^{s}$, and oscillate more and more wildly as one gets closer to the fixed point $\bar{x}$. A similar statement holds for the stable manifold.

\section{Fiqure $0.7 . a$}

Fiqure 0.7 .6

One should expect complex dynamics to occur in such a case. If one considers a small tubular neighborhood $V$ of the stable manifold, the image $G^{n}(V)$ should be, for $n>0$ large enough, a tubular neighborhood of the unstable manifold, as in Figure D.7.b. Intuitively, all the qualitative features of the previous horseshoe example should also be present here. Indeed

Theorem 0.2 .4 (Smale homoclinic theorem) : Let $G$ be a $C^{\prime}$ diffeomorphism of the plane and $\bar{x}$ a hyperbolic fixed point. If the stable and unstable manifolds of $\bar{x}$ intersect transversely at $y \neq \bar{x}$, there exists a neighborhood $V$ of the fixed point and an integer $n>0$ such that the restriction of $G^{n}$ to $V$ is topologically conjugate to the horseshoe map.

The same phenomenon will occur in particular when a family of diffeomorphisms $G_{\alpha}$ of the plane, undergoes a (global) homoclinic bifurcation, i.e. When the unstable manifold of a hyperbolic fixed point becomes tangent (in fact at infinitely many points) to the stable manifold, say when $\alpha=0$, to lead a transverse crossing for $\alpha>0$. The qualitative features of such a bifurcation are described in Figure D.8.a, b, and $c$. 

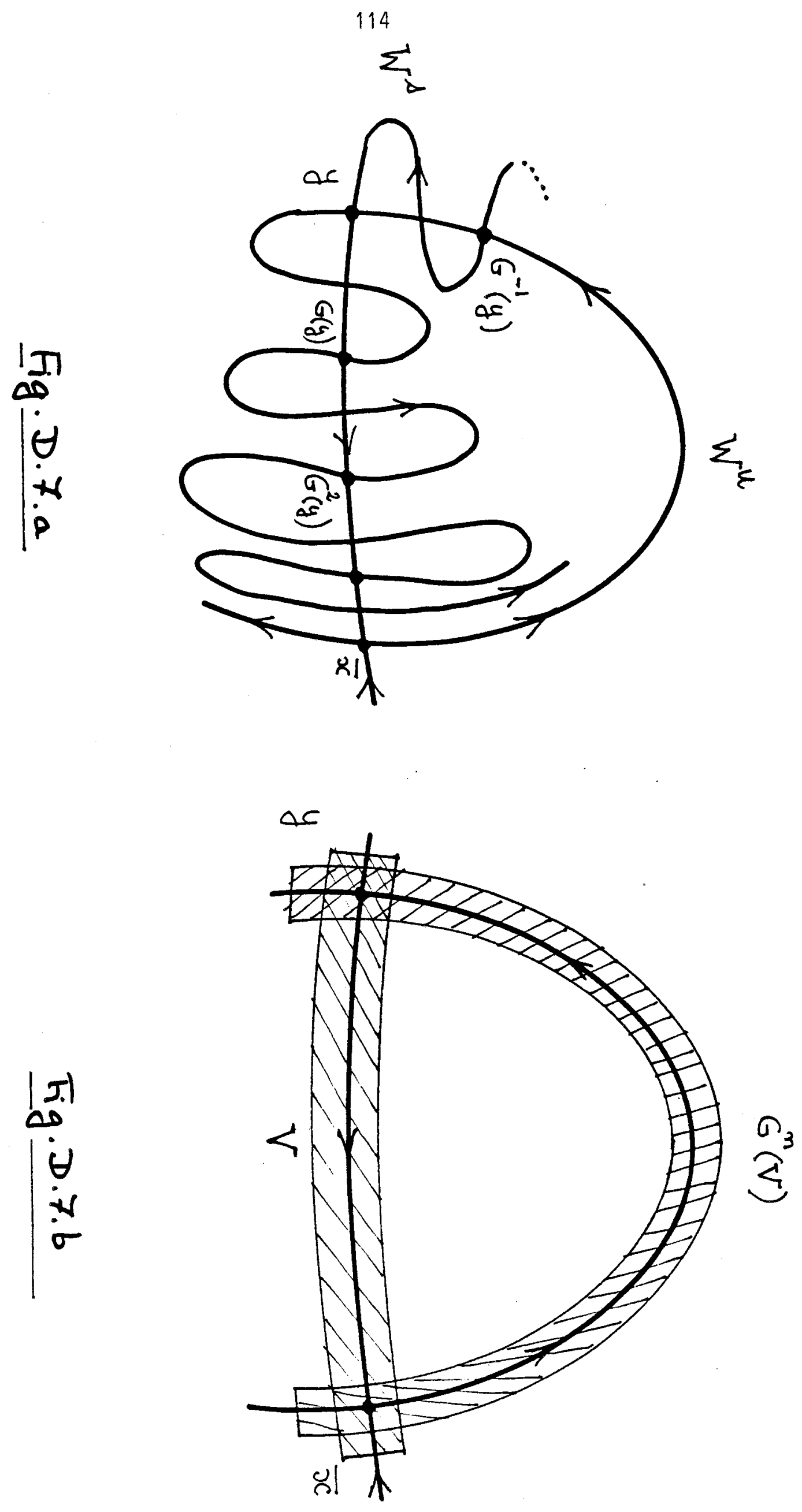


\section{Figure $0.8 . \mathrm{a} \quad$ Figure $0.8 . \mathrm{b}$ Fiqure $0.8 . \mathrm{C}$}

\section{Notes on the literature}

The presentation of the global bifurcations of maps of the real line, in section 0.1 , is adapted from a previous survey, Grandmont (1986), which was itself derived from Collet and Eckmann (1980). See also Guckenheimer and Holmes (1983, Chaps. 5.6 and 6.3), May (1976), Misiurewicz (1983).

Section 0.2 on the horseshoe map and the homoclinic theorem is adapted from the very clear exposition of Lanford (1983), and from Guckenheimer and Holmes (1983, Chap. 5.1 and Theorem 5.3.5); see also Smale (1980).

The use of symbolic dynamics to describe complex orbits is pervasive in this context. For further information and references on this question, the reader may consult Guckenheimer and Holmes (1983, Chap. 5).

The complex invariant sets that we met when studying maps of an interval that have a continuous invariant measure, or when analyzing the horseshoe map, are examples of what is called a strange attractor in the literature. There are many vaiations in the definition of such an object, but the general idea is to define it as a closed invariant set $\wedge$ with a complicated structure, that attracts a set of points having positive Lebesgue measure, and that contains a dense orbit. The requirement that $\wedge$ has a complicated structure is sometimes expressed by the property that it has positive Lebesgue measure, or that its "dimension" is not an integer (fractal dimension), or both. In many instances, one requires the underlying map $G$ to have an ergodic invariant probability measure concentrated on the attractor. One can then define Lyapounov exponents 

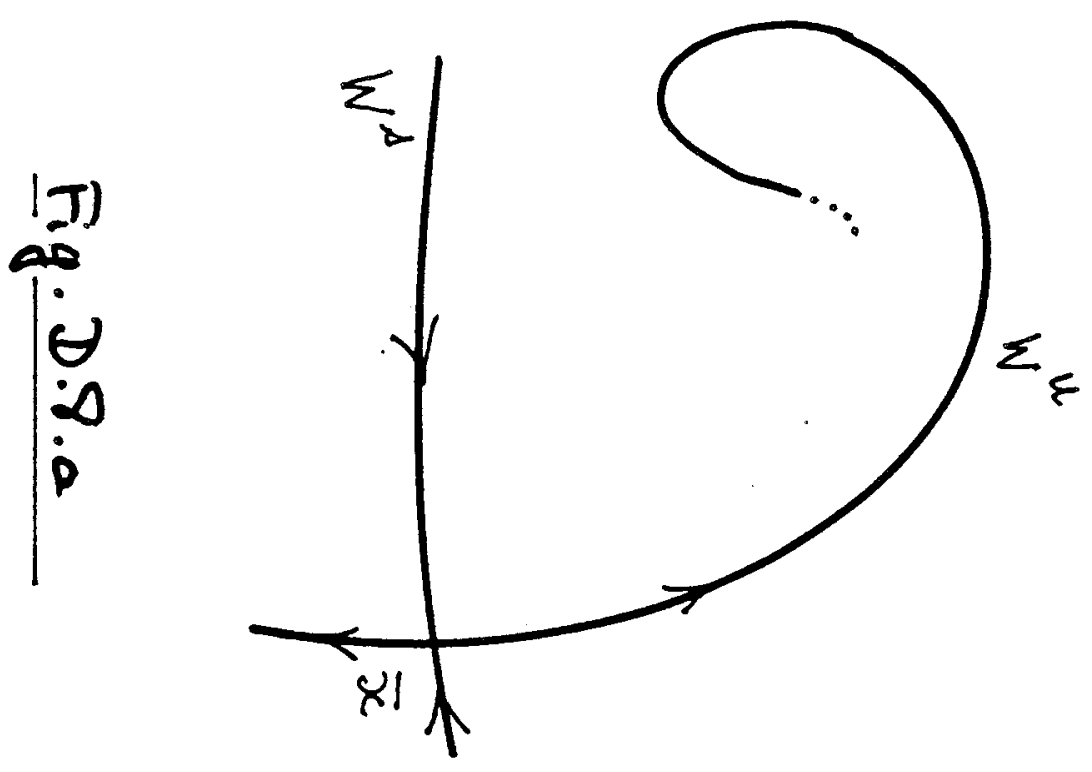

$\left[\begin{array}{l}J] \\ 0 \\ 0 \\ 0 \\ 0\end{array}\right.$

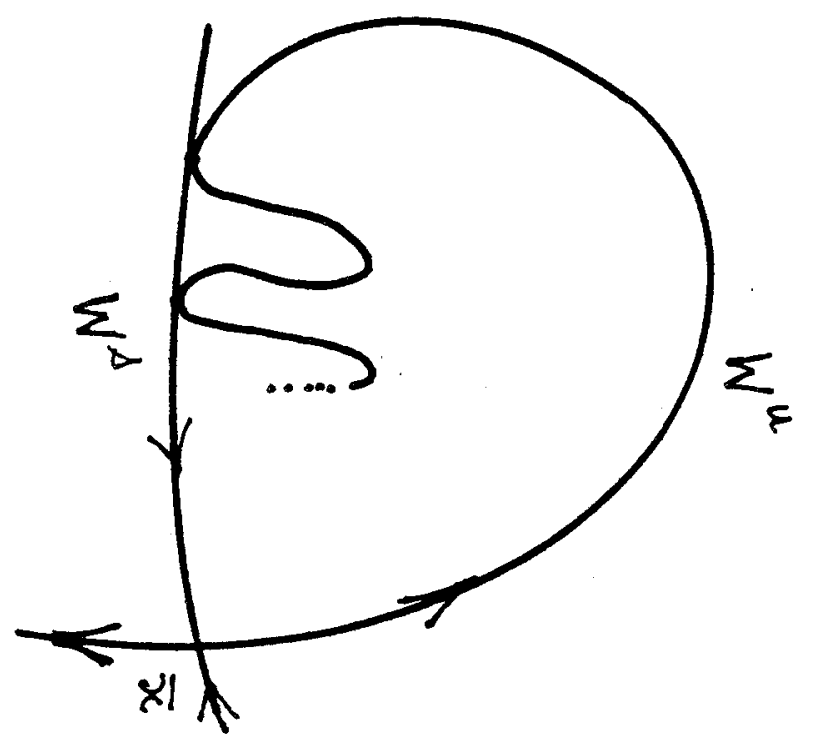

10.1
0
$\dot{\theta}$
$\dot{\alpha}$

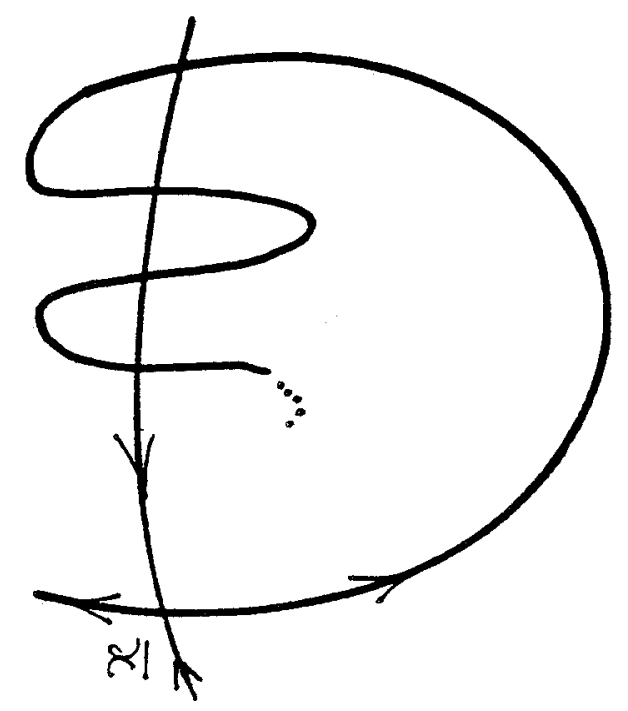


in much the same way as in section D.1. "Turbulence" will then obtain on $\wedge$ if the maximal Lyapounov exponent on $\Lambda$ is positive (the idea of turbulence is also sometimes expressed by the requirement that the attractor contains a transversal homoclinic orbit). On these questions, the reader may consult Guckenheimer and Holmes (1983, Chap. 5). 
Carr, J. (1981), Applications of Centre Manifold Theory, Springer, New York. Chenciner, A. (1983), "Bifurcations de diffeomorphismes de $R^{2}$ au voisinage d'un point fixe elliptique", in Iooss, G., Helleman, R.H.G. and R. Stora (eds.), 1983.

Chenciner, A. (1987), "Bifurcations locales de diffeomorphismes de $R^{n}$, mimeo. Collet, P. and J.P. Eckmann (1980), Iterated Maps on the Interval as Dynamical Systems, Birkhaüser, Boston.

Grandmont, J.M. (1986), "Periodic and Aperiodic Behaviour in Discrete OneDimensional Dynamical Systems", in Hildenbrand, W. and A. Mas-Colell (eds.), 1986.

Guckenheimer, J. and P. Holmes (1983), Nonlinear Oscillations, Dynamical Systems, and Bifurcations of Vector Fields, Springer, New York. Hartman, P. (1964), Ordinary Differential Equations, Wiley, New York. Hildenbrand, W. and A. Mas-Cole11 (1986), Contributions to Mathematical Economics, North-Holland, New York.

Hirsch, M.W. and S. Smale (1974), Differential Equations, Dynamical Systems, and Linear Algebra, Academic Press, New York.

Iooss, G. (1979), Bifurcations of Maps and Applications, North-Holland, New York.

Iooss, G., Helleman, R.H.G. and R. Stora (eds.) (1983), Chaotic Behaviour of Deterministic Systems, North-Hol land, New York. 
Jakobson, M.V. (1981), "Absolute ly Cont inuous Invariant Measures for One-

Parameter Families of One-Dimensional Maps", Comm. Math. Phys. 81, 39-88.

Lanford III, 0.E. (1983), "Introduction to the Mathematical Theory of Dynamical

Systems", in Iooss, G., Helleman, RH.G. and R. Stora (eds.), 1983.

Li, T. and J.A. Yorke (1975), "Period Three Implies Chaos", American

Mathematical Monthly 82, 985-992.

Marsden, J.E. and M. Mccracken (1976), The Hoof Bifurcation and its

Applications, Springer, New York.

May, R.B. (1976), "Simple Mathematical Models with Very Complicated Dynamics", Nature 261, 459-467.

Misiurewicz, M. (1983), "Maps of an Interval", in Iooss, G., Hèlleman, R.H.G. and R. Stora (eds.), 1983.

Palis, Jr., J. and W. de Melo (1982), Geometric Theory of Dynamical Systems, Springer, New York.

Palis, Jr., J. and F. Takens (1977), "Topological Equivalence of Normally Hyperbolic Dynamical Systems", Topology 16, 335-345.

Parthasarathy, K.R. (1967), Probability Measures on Metric Spaces, Academic Press, New York.

Sarkovskii, A.N. (1964), "Coexistence of Cycles of a Continuous Map of the Line into Itse If", Ukr. Mat. Z. 16, 61-71.

Singer, D. (1978), "Stable Orbits and Bifurcations of Maps of the Interval", SIAM J. Applied Math. 35, 260.

Smale, S. (1980), The Mathematics of Time, Springer, New York.

Whitley, D. (1983), "Discrete Dynamical Systems in Dimensions One and Two", Bu11. London Math. Soc. 15, 177-217. 